\title{
FINITE ELEMENT EVALUATION OF ENERGY DISSIPATING STEEL PLATE FUSE LINKS
}

\author{
By \\ Ahmad Siar Mahmood Shah \\ B.Sc. (Civil Engineering), Antalya Bilim Univeristy, Turkey, 2017
}

\author{
A thesis presented to Ryerson University \\ in partial fulfillment of the requirements for the degree of \\ Master of Applied Science \\ in the program of Civil Engineering
}

Toronto, Ontario, Canada, 2020

(C) Ahmad S. M. Shah, 2020 


\section{Author's Declaration}

I hereby declare that I am the sole author of this thesis. This is a true copy of the thesis, including any required final revisions, as accepted by my examiners.

I authorize Ryerson University to lend this thesis to other institutions or individuals for the sole purpose of scholarly research.

I further authorize Ryerson University to reproduce this thesis by photocopying or other means, in total or in part, at the request of other institutions or individuals for the sole purpose of scholarly research.

I understand that my thesis may be made electronically available to the public. 


\section{ABSTRACT \\ Finite Element Evaluation of Energy Dissipating Steel Plate Fuse Links \\ Ahmad Siar Mahmood Shah \\ MASc., Department of Civil Engineering, Ryerson University, 2020}

Steel plate devices are widely used for providing energy dissipation in earthquake resistant structures. The cyclic response of these steel plate fuses varies depending on different design factors related to the fuse link geometry or its material property. This research presents a numerical study on the cyclic response of steel plate fuses with butterfly-shaped and straight shear links. Finite element models are first developed and validated against past experimental studies. A sensitivity study using the design-of-experiment method is performed to statistically determine the effects of different design parameters on the cyclic response of steel plate shear links. Seven design factors related to the material or geometry of steel plate fuses are considered as input factors. The cyclic response of fuses is examined in terms of initial stiffness, yield strength, ultimate stiffness, effective damping, maximum strength, and ductility. From the results of the sensitivity analysis, it is shown that the overall cyclic behavior of steel plate fuses is most significantly influenced by the fuse link end-width and thickness while the fuse link length and mid-width are partially influential on some of the cyclic responses. In addition, an optimization study is performed to determine the optimal ranges for the design factors that result in simultaneous response optimization conditions, such as maximized energy dissipation capability and ductility. Predictive equations are also developed and validated for the cyclic response characteristics of steel plate shear links. Based on the confirmation runs, it is believed that these equations can accurately predict the acquired responses for steel plate fuse links. 


\section{Acknowledgements}

I would like to express my sincere gratitude to my advisor Dr. Saber Moradi for his inspiration, technical and personal insight, and unwavering support throughout this research. His constant guidance, vision, and enthusiasm have helped me to learn and deepen my interests in this topic. His advice and thoughts have always been the greatest source and motivation to make possible the accomplishments herein.

I am grateful to all my friends at Ryerson University. I specifically extend my thanks to Majid M. Nia for his help and advice on ANSYS. I owe my appreciation towards my respected parents and my family for their love and support. 


\section{Dedication}

This thesis is dedicated to my parents and my family. 


\section{TABLE OF CONTENTS}

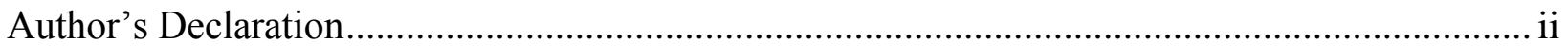

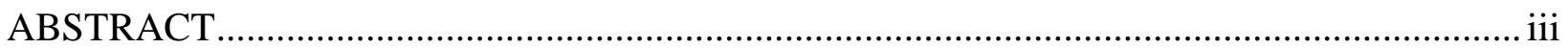

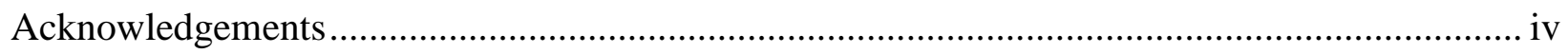

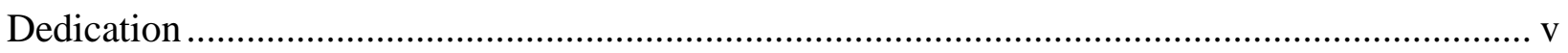

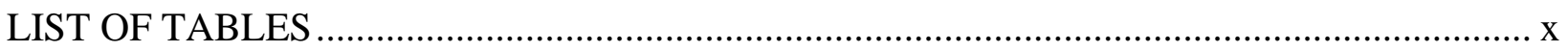

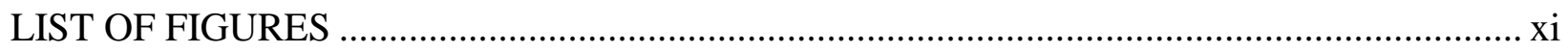

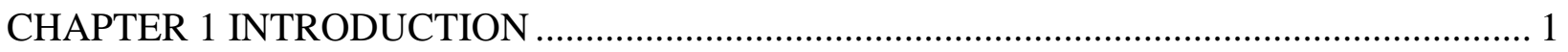

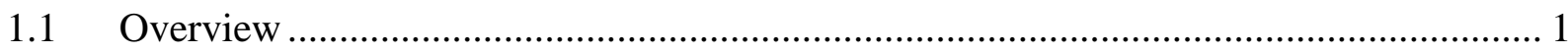

1.2 Research Motivation and Objectives.......................................................................... 3

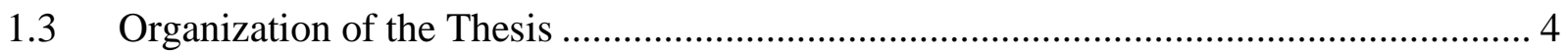

CHAPTER 2 LITERATURE REVIEW ……………......................................................... 5

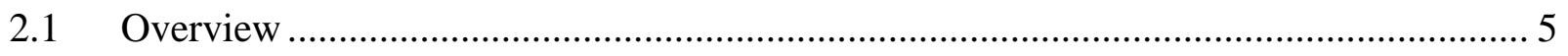

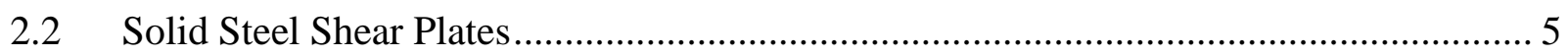

2.3 Straight Steel Shear Plates and Walls ....................................................................... 7

$2.4 \quad$ Hourglass-shaped Steel Shear Plates...................................................................... 15

2.5 Triangularly Shaped Steel Shear Plates ……………………………….................. 19

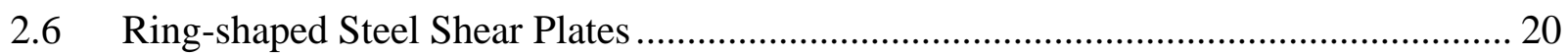

2.7 Honeycomb-shaped (butterfly-shaped) Steel Shear Plates .......................................... 21

2.7.1 Low Cycle Fatigue Test on Butterfly-shaped Steel Shear Plates .......................... 25

2.7.2 Cyclic Performance of Non-uniform Butterfly-shaped Steel Shear Plates............. 27

2.7.3 Shape Investigation on Butterfly-shaped Steel Shear Plates .................................. 28

2.7.4 Parametric Studies of Butterfly-shaped Steel Shear Plates with Various

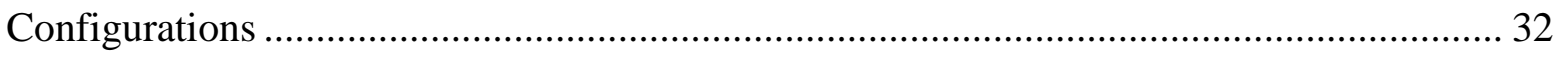

2.7.5 Investigating Limit States for Butterfly-shaped Steel Shear Plates ........................ 33 
2.7.6 Yielding and Lateral Torsional Buckling Limit States of Butterfly-shaped Steel

Shear Plates. 34

2.7.7 Performance of Butterfly-shaped Shear Panels ............................................... 34

2.8 Comparison of Butterfly-shaped Steel Shear Plates to Slits .................................... 35

2.9 Effect of Yield Strength on Steel Shear Plates.................................................... 35

2.10 Design and Behavior of Steel Shear Plates ...................................................... 35

2.11 Potential Applications of Structural Steel Shear Plates .......................................... 36

2.11.1 Steel Shear Plates in Rocking Frames ..................................................... 36

2.11.2 Eccentrically Braced Steel Frames ................................................................. 37

2.11.3 Use of Steel Shear Plates in Bridges ............................................................ 38

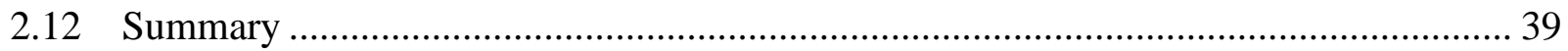

CHAPTER 3 FINITE ELEMENT (FE) MODELING ..................................................... 40

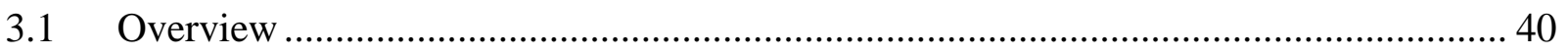

3.2 Reference Slit and Butterfly-shaped Steel Plate Fuses ........................................ 40

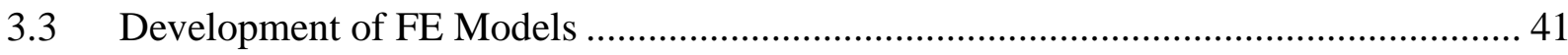

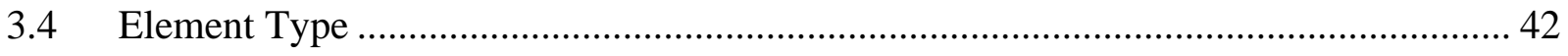

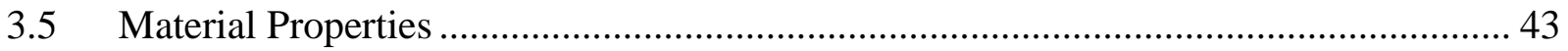

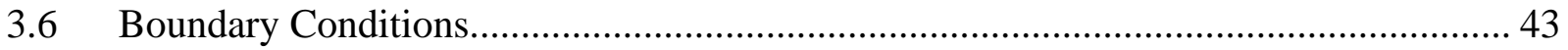

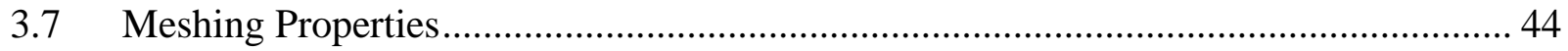

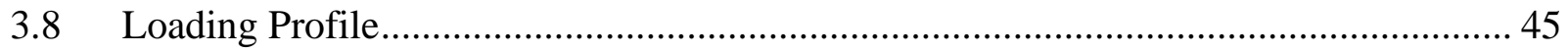

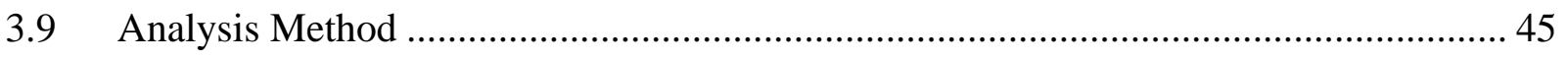

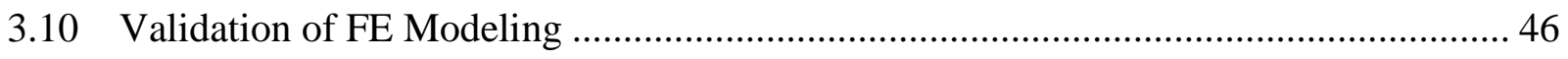

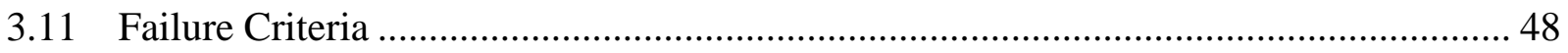

CHAPTER 4 SENSITIVITY ANALYSIS OF ENERGY DISSIPATING STEEL PLATE FUSES 51

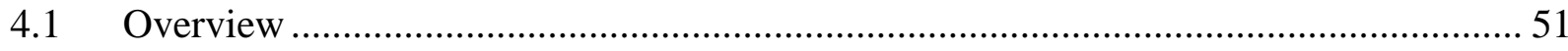




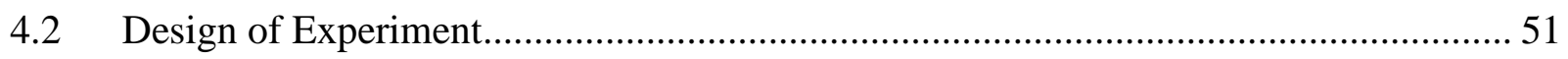

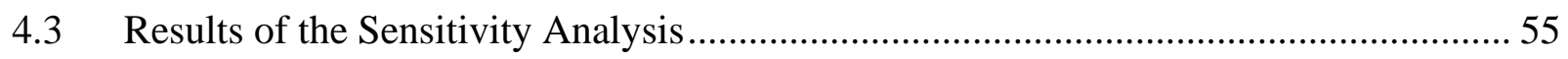

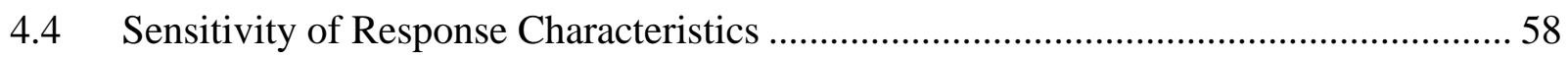

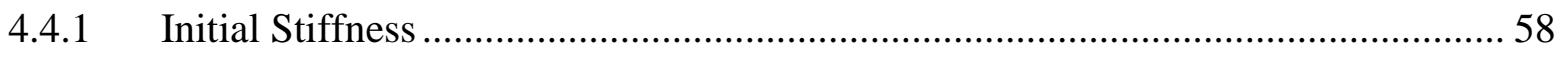

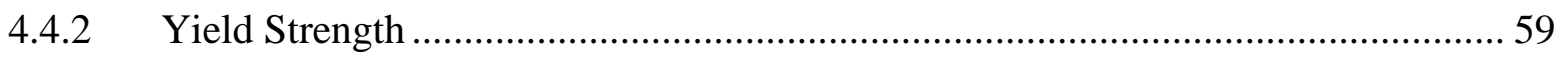

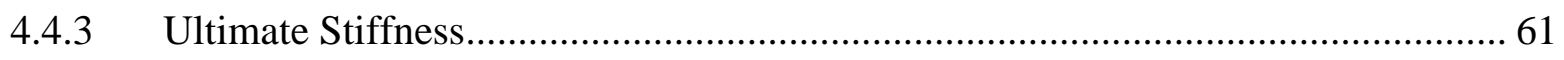

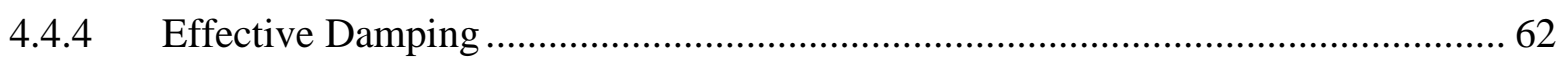

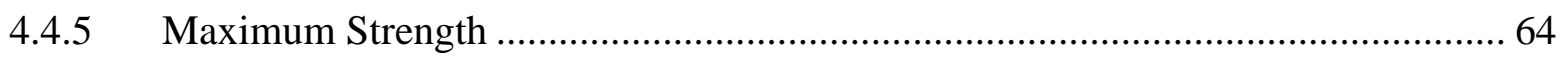

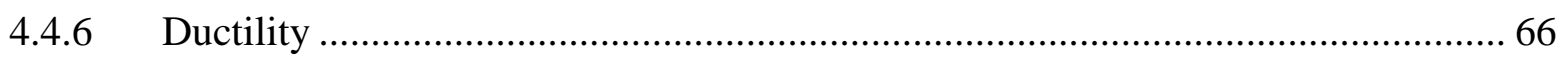

CHAPTER 5 PREDICTIVE MODELS FOR ENERGY DISSIPATING STEEL PLATE FUSES

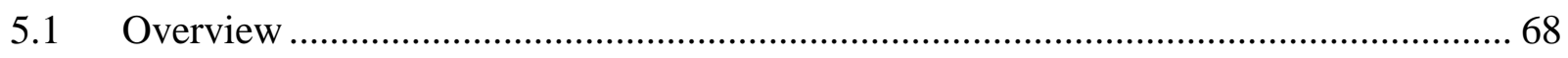

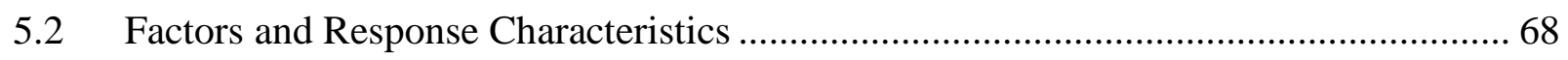

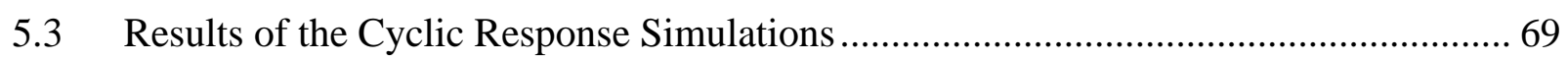

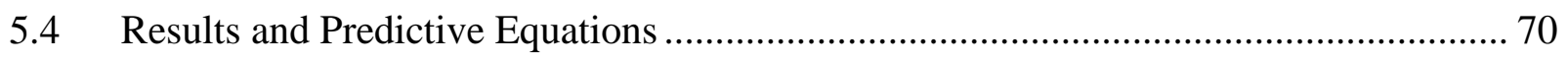

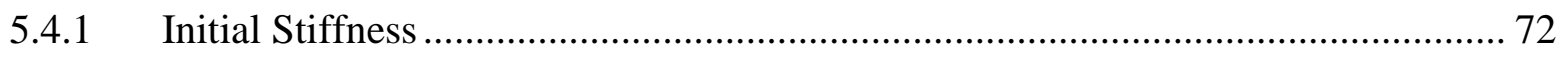

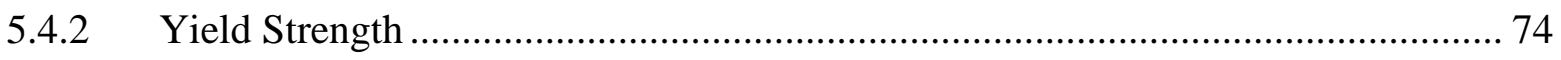

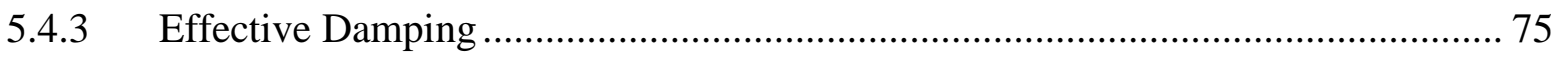

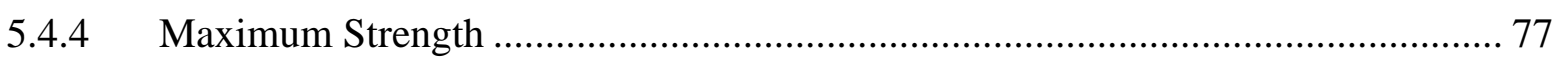

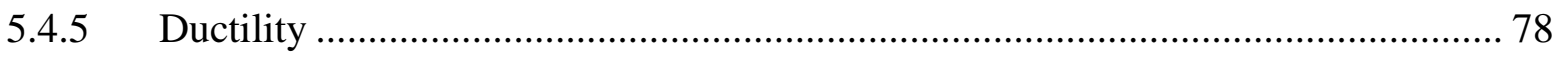

5.5 Verification of the Response Surface Models .............................................................. 80

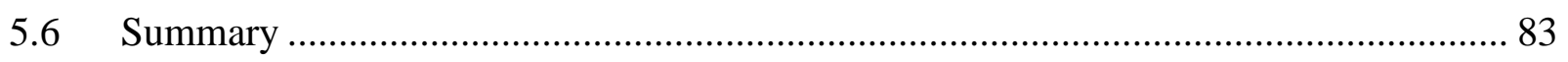

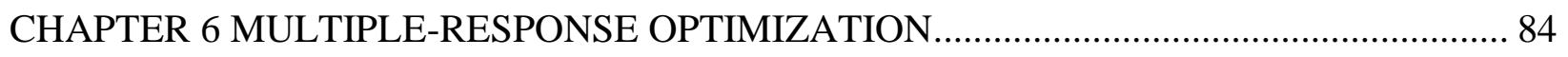

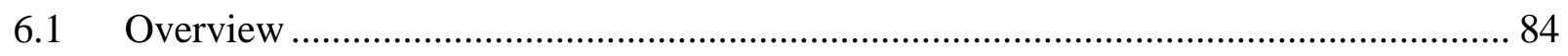

6.2 Desirability Functions for Multiple-response Optimization .......................................... 84 


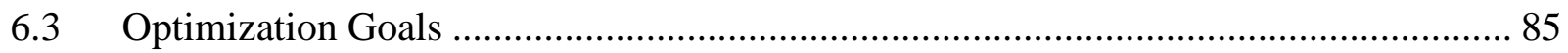

6.4 Results and Discussions of the Optimization Studies ........................................... 86

6.4.1 Results of Optimization Problem 1 1............................................................. 86

6.4.2 Results of Optimization Problem 2 ........................................................... 89

6.4.3 Results of Optimization Problem 3 ........................................................... 94

6.4.4 Results of Optimization Problem 4 .......................................................... 97

6.5 Confirmation of the Optimization Results ....................................................... 101

CHAPTER 7 SUMMARY AND CONCLUSIONS ....................................................... 103

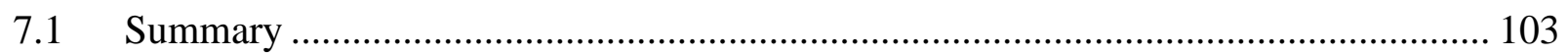

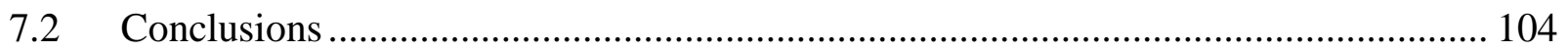

7.2.1 Cyclic Response Sensitivity of Energy Dissipating Steel Plate Fuses ............... 104

7.2.2 Predictive Equations and Multiple-response Optimization .............................. 105

7.3 Future Recommendations........................................................................... 106

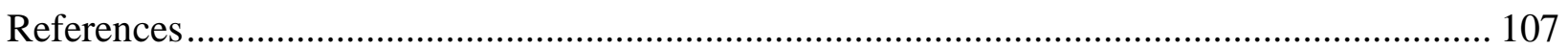




\section{LIST OF TABLES}

Table 3. 1 Dimensional details of the specimens used for the validation study $[15,16]$........... 41

Table 4. 1 Factors and ranges considered for the sensitivity analysis. .................................... 52

Table 4. 2 Factor combination for each model. ..................................................................... 53

Table 4. 3 Response characteristics of each model........................................................... 54

Table 4. 4 Percentage Contribution of important factors and interactions for each response

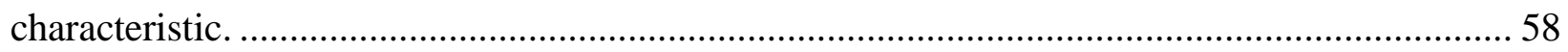

Table 5. 1 Factors and ranges considered for the response surface study. ................................ 68

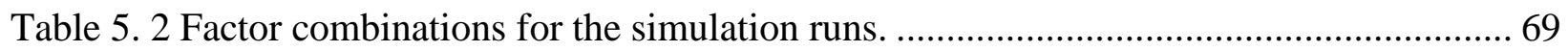

Table 5. 3 Response characteristics of each model........................................................... 71

Table 5. $4 \beta i$ values for all the response variables. ........................................................... 72

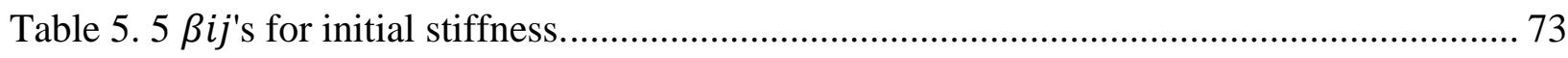

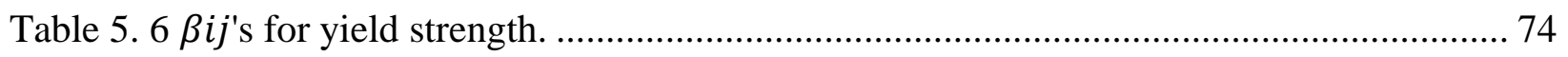

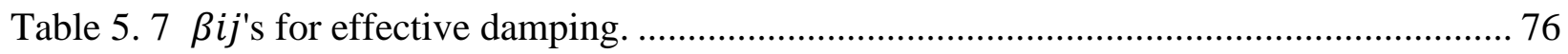

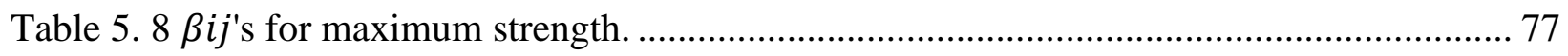

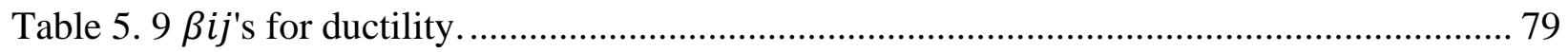

Table 5. 10 Factor combinations used for the verification of the response surface models........ 80

Table 5. 11 Response values for the verification of response surface models. ........................ 81

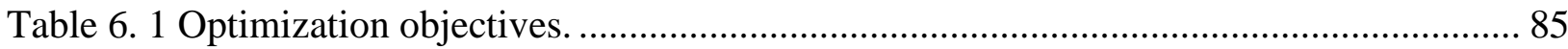

Table 6. 2 Ranking of the most influential input parameters to least for optimization problem 1.

Table 6. 3 Ranking of the most influential input parameters to least for optimization problem 2.

Table 6. 4 Ranking of the most influential input parameters to least for optimization problem 3.

Table 6. 5 Ranking of the most influential input parameters to least for optimization problem 4. 100

Table 6. 6 Confirmation of the optimization results. 102 


\section{LIST OF FIGURES}

Figure 1. 1(a) Steel plate fuses with slit or straight cut openings and (b) Butterfly-shaped steel

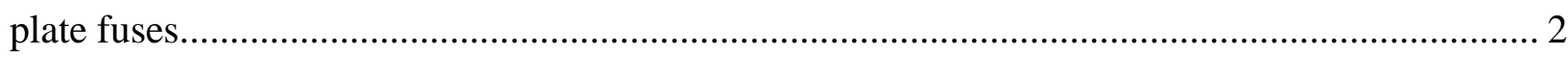

Figure 2. 1 Experimental test done by Ji et al. [adopted from [17]] ........................................ 5

Figure 2. 2 Steel shear links are used in the beam section [adopted from [18]] ............................. 6

Figure 2. 3 The steel shear plate detail in beam connections [adopted from [18]] ......................... 7

Figure 2. 4 Straight steel shear wall example [adopted from [19]] .......................................... 8

Figure 2. 5 Straight shear links with stiffeners [adopted from [17]] ............................................ 9

Figure 2. 6 Use of straight shear links in an inverted V braced system [from [22]]..................... 11

Figure 2. 7 Straight shear links used by Oh et al. [23]........................................................... 12

Figure 2. 8 Use of steel slit shear links in beam to column connections [ from [24]] .................. 13

Figure 2. 9 Test of steel slit dampers: (a) Observance of fracture in the specimen; (b) Hysteresis

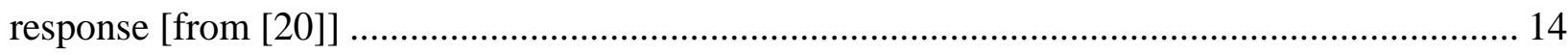

Figure 2. 10 Test of steel shear walls with cuts: (a) Tested Specimen; (b) Hysteresis response

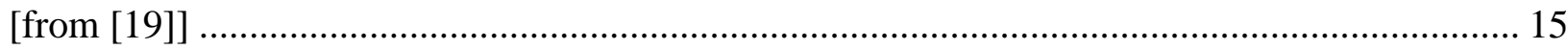

Figure 2. 11 Shifting the location of hinge away from connection [from [26]] .......................... 16

Figure 2. 12 Perimeter perforated model proposed by Koppal et al. [from [27]]........................ 17

Figure 2. 13 Steel sheet wall with circular holes [adopted from [28]] ......................................... 18

Figure 2. 14 Use of ribs on the Circular holes [from [28]] ……............................................ 19

Figure 2. 15 Implementation of triangular shaped shear links in masonry structures [from [29]] 20

Figure 2. 16 Experimental test of Honeycomb dampers: (a) Tested specimen, (b) Hysteresis

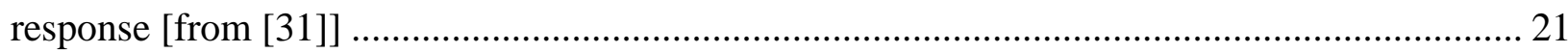

Figure 2. 17 Hysteretic Dampers [from [31]] …………............................................... 22

Figure 2. 18 Utilization of Butterfly-shaped links in wall systems [from [31]] ......................... 23

Figure 2. 19 Added damper and stiffness (ADAS) Concept [from [32]] .................................... 24

Figure 2. 20 The effect of ADAS on the hysteresis response of the frame [from [32]] ............... 25 Figure 2. 21 Experimental Tests of ADAS elements: (a) specimen's section perpendicular to the direction of loading; (b) Specimen's section parallel to the direction of loading; (c) Hysteresis

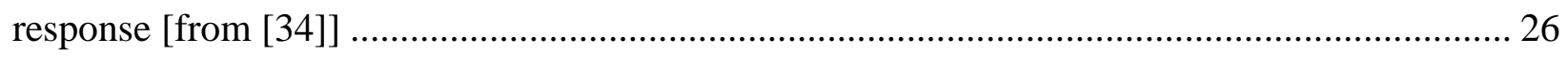

Figure 2. 22 Different shapes investigated in the study of $\mathrm{Li}$ and $\mathrm{Li}$ et al. [35] ......................... 29

Figure 2. 23 Hysteresis response of Butterfly-shaped specimens [from [35]] ............................ 29 
Figure 2. 24 Different purposed dampers [from [14]] ................................................. 31

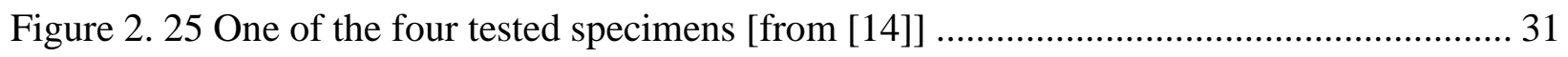

Figure 2. 26 Dimensions of the recommended specimen [from [14]] .................................... 32

Figure 2. 27 Implementation steel shear plates in different structures [from [16]] ................... 36

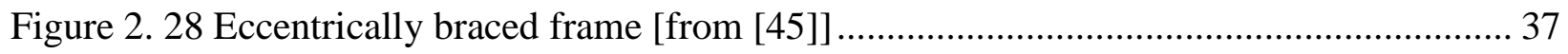

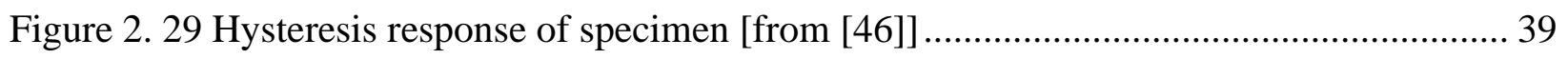

Figure 3. 1 Details of PSD-5 specimen used for the validation study (all dimensions are in $\mathrm{mm}$ )

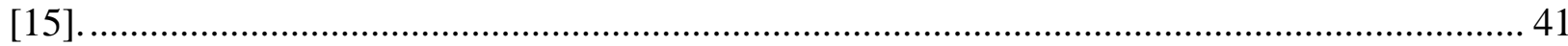

Figure 3. 2 Details of DSD-5 specimen used for the validation study (all dimensions are in $\mathrm{mm}$ )

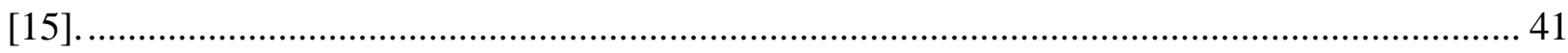

Figure 3. 3 Finite element model for PSD-5 specimen.................................................. 42

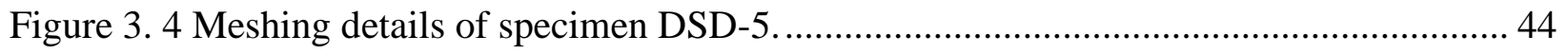

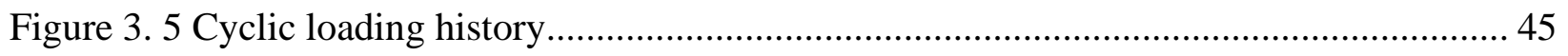

Figure 3. 6 Cyclic load-displacement response of specimens DSD-5 and PSD-5: FE simulation results in comparison with experimental results.

Figure 3. 7 Validation of the finite element modeling against a numerical reference study of limit states [16]: (a): flexural yielding, (b): shear yielding, (c): lateral torsional buckling................. 47

Figure 4. 1 Hysteretic response plots for: (a) model 1; (b) model 7; (c) model 12................... 56

Figure 4. 2 The effect of interaction between the thickness and the end-width on initial stiffness.

Figure 4. 3 The effect of interaction between the end-width and the thickness on yield strength.

Figure 4. 4 The effect of interaction between the length and the thickness on ultimate stiffness. 62

Figure 4. 5 The effect of interaction between the end-width and the thickness on effective damping

Figure 4. 6 The effect of interaction between the end-width and the thickness on maximum strength.

Figure 4. 7 The effect of interaction between the thickness and the length of the link on ductility. 
Figure 5. 1 Hysteretic response plots for: (a) model 2, (b) model 4, (c) model 8, and (d) model 13. 70

Figure 5. 2 Variation of initial stiffness with respect to different pairs of design factors. .......... 73

Figure 5. 3 Variation of yield strength with respect to different pairs of design parameters. ...... 75

Figure 5. 4 Variation of effective damping with respect to different pairs of design factors....... 76

Figure 5. 5 Variation of maximum strength with respect to different pairs of input parameters. 78

Figure 5. 6 Variation of ductility with respect to different pairs of input parameters. ................ 79

Figure 5. 7 Comparison of actual response with predicted response using response surface

models

Figure 6. 1 The variation of desirability function versus input parameters for optimization problem 1. 87

Figure 6. 2 Contour plots of desirability functions for optimization problem 1 89

Figure 6. 3 The variation of desirability function versus input parameters for optimization problem 2. 91

Figure 6. 4 Contour plots of desirability functions for optimization problem 2 ........ 93

Figure 6. 5 The variation of desirability function versus input parameters for optimization problem 3. 95

Figure 6. 6 Contour plots of desirability functions for optimization problem 3 .........

Figure 6. 7 The variation of desirability function versus input parameters for optimization

problem 4.

Figure 6. 8 Contour plots of desirability functions for optimization problem 4 101 


\section{CHAPTER 1 INTRODUCTION}

\subsection{Overview}

The conventional approach to seismic design is based on ductile behavior of structures. Appropriate strength and inherent ductility of structures allow inelastic drift capacity and energy dissipation during severe seismic motions. Steel plates with engineered cuts can be implemented in the structures as the sacrificial ductile structural elements and easily replaceable fuses. The fuse term is referred to any element in which the damage caused by earthquake is concentrated on it, thus after every severe event of earthquake this element is replaced [1,2]. By confining the inelastic deformation and structural damage to steel plates with shear links, the primary structural elements like beams and columns remain essentially elastic and undamaged. One example of using structural steel plate fuses is in controlled rocking steel braced frames, which have been shown as promising self-centering systems that can minimize financial losses due to repair costs and downtime in structures under strong earthquakes [3].

The inelastic deformation of energy dissipating devices is an essential mechanism for input earthquake energy dissipation. In addition, steel plate device or fuse element that is accessible and easily replaceable is also a popular and inexpensive choice for energy dissipation because of its relatively sufficient ductility and high potential for energy dissipation in the post-yield region. The damping, ductility, and reusability of steel have made it ideal for wide applications in energy dissipating devices (e.g., [4]). Particularly, developments of self-centering rocking frames rely on the energy dissipation of steel shear plate fuses (e.g., [3]).

The idea of utilizing steel plate fuses in structures to absorb a large portion of the seismic energy has first begun with the conceptual and experimental work by Kelly et al. [5]. Then several studies have investigated various types of energy dissipating steel plate fuses (e.g., [6]). After a series of theoretical and experimental research work, more and more interests and attentions are turned to the application of steel plate fuses [7,8].

Numerous studies have examined the energy dissipation and hysteretic response of steel shear plates and dampers. Among others, Ma et al. [9] has experimentally tested butterfly-shaped fuses (with diamond-shaped openings) and slit fuses (with rectangular openings). These fuses are 
shown in Figure 1.1. By making openings or cuttings in the plate, the plate's buckling and ductile behavior are enhanced since a flexural mode may govern, as opposed to a single plate's prevailing shear mode response. Further, an individual shear link is more compact than a solid plate. Chan et al. [10] developed and tested a new steel slit damper (SSD). They reported that the device exhibited stable hysteresis with excellent energy dissipation and ductility. Ghabraie et al. [11] found that diamond-shaped cuts were optimal based on a topology optimization of SSDs in terms of energy dissipation. Deng et al. [12] studied the shape optimization of steel shear panel dampers by minimizing the maximum equivalent plastic strain in simulated models.

The importance of steel plate fuse links acquires a comprehensive study on the effects as well as the interaction effects of each design parameter included in the fuse plate. As mentioned above, these plates play a significant role in reducing the damage in the main structural components by providing sufficient energy dissipation and ductility in any event of seismic motion. Further the effects of design factors in steel plate fuse links on other structural response characteristics such as yield strength, initial stiffness, ultimate stiffness and maximum strength should also be thoroughly investigated.

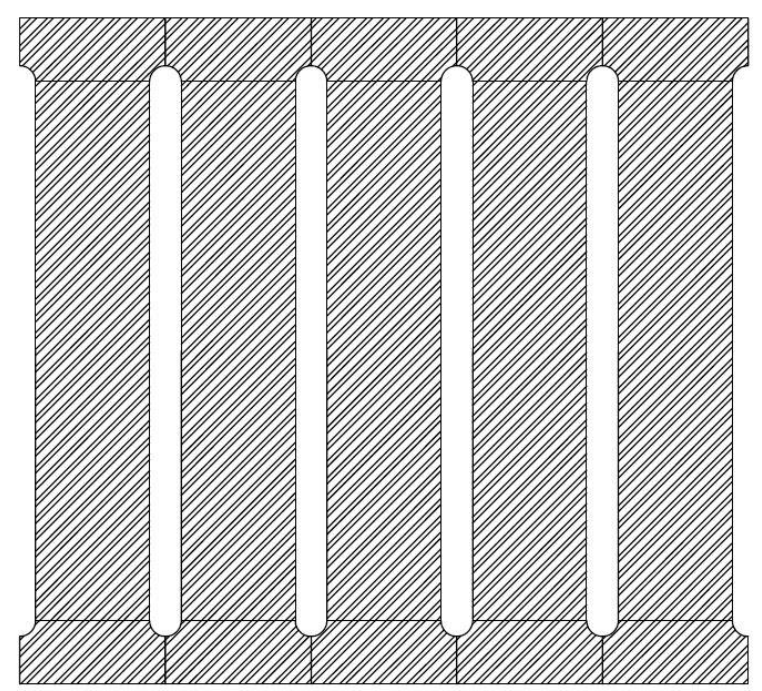

(a)

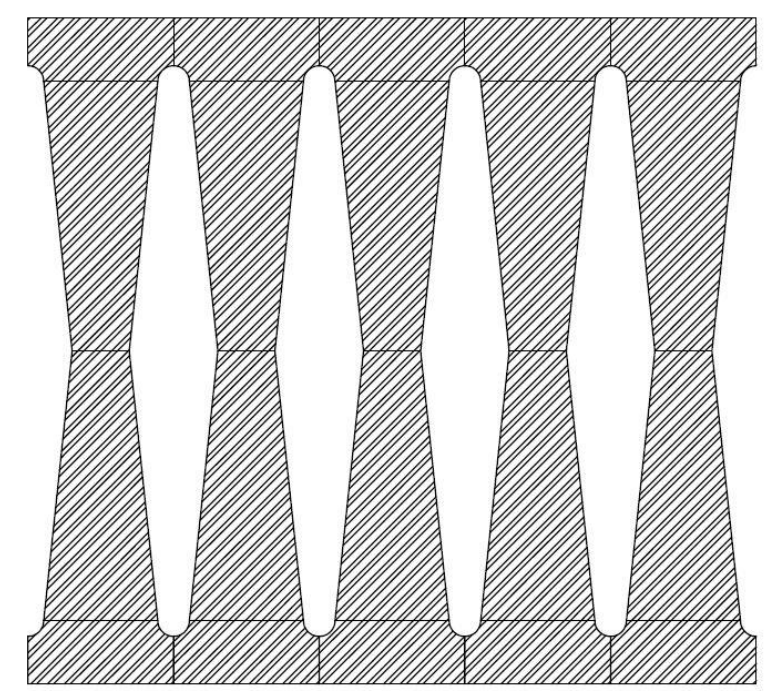

(b)

Figure 1. 1(a) Steel plate fuses with slit or straight cut openings and (b) Butterfly-shaped steel plate fuses. 


\subsection{Research Motivation and Objectives}

In this research, the sensitivity of the cyclic response of steel plate fuses to different design parameters is assessed. In previous studies (e.g., $[13,14]$ ) the variability of the fuse cyclic response to a number of individual parameters and their ratios such as thickness, end-width to thickness, length to thickness, mid-width to end-width of steel plate fuses has been addressed. However, there is no previous statistical study of main factor and interaction effects on the fuse cyclic response.

In the present thesis, a statistical design of experiment method is used to examine the effect of each parameter by changing different design parameters together. Furthermore, possible interactions between design parameters are determined. The presence of an interaction between the two factors is statistically indicated when the response sensitivity for one factor varies at different levels of another factor.

In this study, first, a detailed finite element model that could extend the research on steel plate fuses is generated to accurately capture the response of steel plate specimens against results of past experimental and numerical studies $[15,16]$. Following that, a two-level factorial design with seven factors that potentially influence the fuse cyclic response characteristics is considered. The selected factors are related to either the fuse geometry or its material. The geometry related factors include the fuse link length, mid-width, end-width, thickness, and band zone length. The

effect of the fuse yield strength and modulus of elasticity as material-related factors are also evaluated. Thirty-two finite element models of fuses with different design factor combinations are analyzed and the cyclic force-displacement response of each steel plate fuse is assessed in terms of initial stiffness, yield strength, ultimate stiffness, effective damping, maximum strength, and ductility.

Following the sensitivity study, the cyclic response characteristics of steel plate fuses are optimized based on validated finite element modeling and a statistical response surface method. From the results of the sensitivity study, four geometry-related parameters are considered as the most influential factors including: the length, mid-width, end-width, and thickness. A response surface methodology is used to predict the response variables. Response optimization studies are then performed using a desirability approach. The accuracy of the predictions and optimization results is next verified by performing confirmation studies. 


\subsection{Organization of the Thesis}

- Chapter 2 provides a detailed literature review of past research related to energy dissipating steel plate fuses.

- Chapter 3 describes the finite element modeling techniques used in this study. The finite element modeling which includes the explanation of modeling procedure, appropriate selection of element type, material properties, boundary conditions, meshing properties, loading profile and the analysis method, is thoroughly elaborated. This chapter also includes the validation of finite element analysis using experimental tested results on energy dissipating steel plate fuses.

- Chapter 4 describes the results of a cyclic response sensitivity study of energy dissipating steel plate fuses. The sensitivity study is conducted in a design of experiment framework. The study also determines the importance of four different factors.

- Chapter 5 derives the predictive equations for response characteristics of energy dissipating steel plate fuses. These response variables include initial stiffness, yield strength, ultimate stiffness, effective damping, maximum strength, and ductility. The response surface methodology is used to predict the response parameters in terms of significant factors identified in Chapter 4.

- Chapter 6 studies multiple-response optimization of energy dissipating steel plate fuses. Four sets of objectives are defined in the multiple-response optimization.

- Chapter 7 summarizes the major findings and conclusions of this work. Recommendations for future research are also provided. 


\section{CHAPTER 2}

\section{LITERATURE REVIEW}

\section{$2.1 \quad$ Overview}

This chapter reviews a detailed past research pertinent to the topic of energy dissipating steel plates (steel shear plates). Different shapes of steel shear plates including solid, straight, hourglass-shaped, triangularly, ring and honeycomb-shaped are thoroughly described. Later, possible comparisons of these steel plates are made in terms of structural design and behavior. Following that, potential applications of structural steel shear plates are briefly reviewed.

\subsection{Solid Steel Shear Plates}

Solid plate shear links have been used in various applications for seismic resistance purposes. A new set of short shear links (Figure 2.1) which are used for coupling beams and have the replaceability advantage after every significant damage have been proposed by Ji et al. [17]. These short shear links are primarily designed for eccentric braced frame systems having lower yielding strengths to ensure the capability of energy dissipation under high seismic loading conditions. However, the main concern in using these shear links is the high over strength factor which leads to significant boundary element sizes.

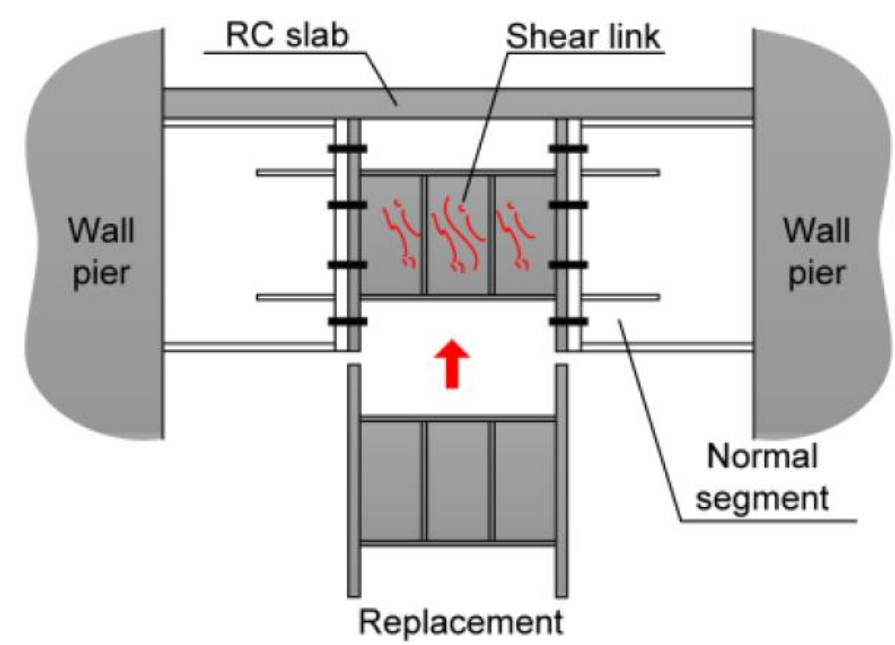

Figure 2. 1 Experimental test done by Ji et al. [adopted from [17]].

The over strength factor values are suggested to be less than 1.5 according to AISC 341 , while the studied short links by Ji et al. [17] has reached 1.9. However, the plastic rotation capacity, 
which is defined as the ability to maintain at least $80 \%$ of the maximum strength in the test, is found approximately $0.15 \mathrm{rad}$. According to AISC 341 this value is $0.08 \mathrm{rad}$. Additionally, the effect of stiffener is investigated, and it has been observed that stable hysteric response, as well as inelastic deformation capacity can be achieved, if the stiffener spacing requirements proposed by the codes are met. Having said that, it does not mean that there would be a significant change in the overall response if the spacing criteria is $50 \%$ more than what has been suggested in the code requirements for spacing. It is worth to mention that the overstrength value is estimated by dividing the maximum value of shear over the designed shear value which is the smaller value of either shear or flexure obtained from the equations given by AISC 360.

Along the same lines, Castiglioni et al. [18] have studied the implementation of solid shear links in composite steel frames. The conducted study by Castiglioni et al. [18] has investigated the behavior of the two types of structures under seismic loading, in which the damage would be concentrated on the solid shear links. The solid shear links limit the damage on the surrounding structural members and can be replaced after being damaged. The links connecting the beam to column connection are shown in Figure 2.2.

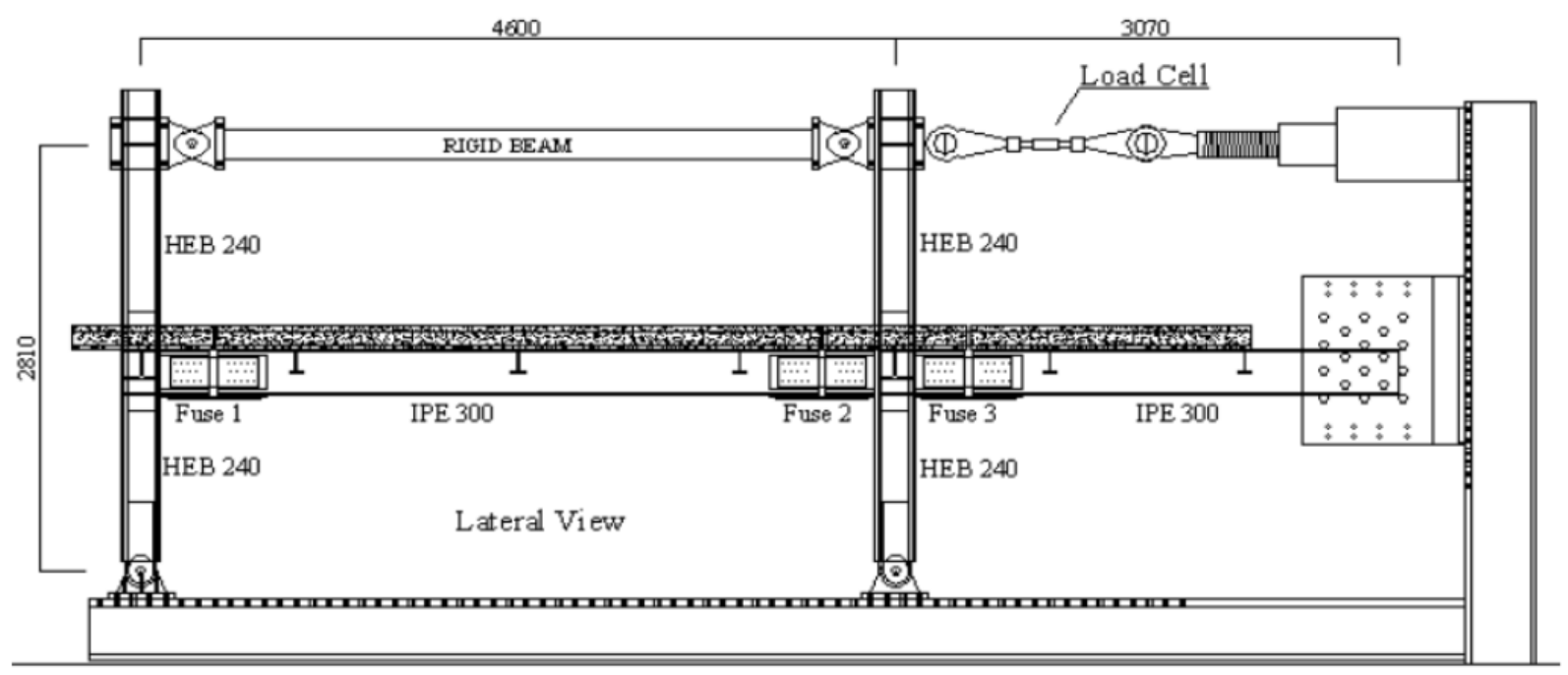

Figure 2. 2 Steel shear links are used in the beam section [adopted from [18]]

The arrangement of connections which are shown in Figure 2.3, shifts the center of rotation at the solid shear link steel plate above, and it stays in between the two reinforcement layers. As a result, the steel plates in the fuse devices can be easily buckled and deformed; thus, dissipating 
significant amount of energy without damaging the surrounding structural components. It is worth to mention that the reinforced concrete slab does not get a significant damage due to large story drifts, which causes large rotations in the solid steel shear plates.

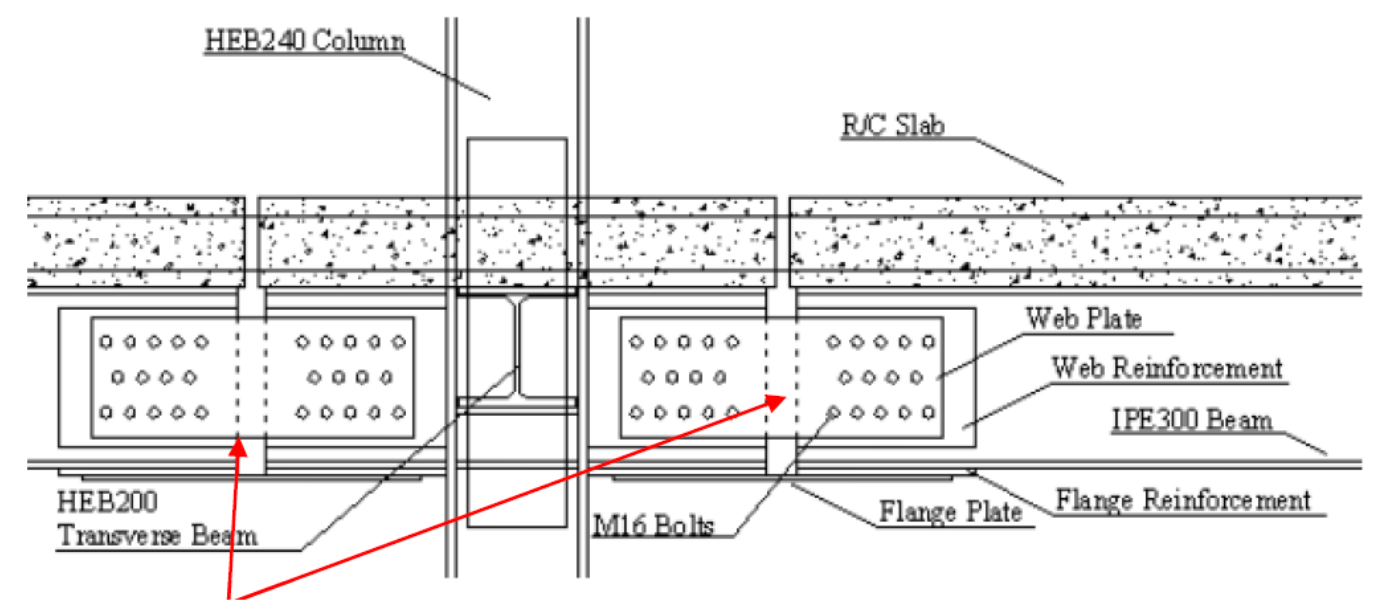

Rectangular links

Figure 2. 3 The steel shear plate detail in beam connections [adopted from [18]]

\subsection{Straight Steel Shear Plates and Walls}

The steel straight panels are common systems that have been used as the lateral resisting systems due to energy dissipation capacity, simplicity in installation, potential for architectural flexibility and seismic retrofitting. When the steel straight panels are subjected to lateral horizontal shear loads or displacements, the links in the panel act as beams in double curvature, dissipating energy in flexure. These straight panels are well known as the first passive device used for dissipation of energy in buildings as shown in Figure 2.4 ([19]). 


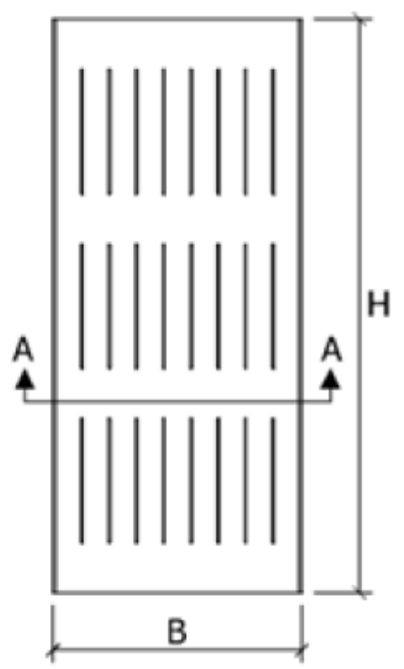

Figure 2. 4 Straight steel shear wall example [adopted from [19]]

It is mentioned that straight plates having openings or cuttings in it, would dissipate higher amount of energy when compared to the corresponding solid plates [19]. Straight shear panels show more flexibility and govern the flexural mode behavior compared to the predominated shear mode behavior of the original solid plates. Furthermore, the buckling of the shear plate can be controlled by the width over thickness ratio. An increase in the width over thickness ratio significantly improves the buckling resistance of steel shear panels. However, there are certain issues associated with slit linked shear plates such as; low cycle fatigue and large over strength factor [20]. Previous studies show that a fixed width to thickness ratio of 10, can result in at least $2.5 \%$ drift ratio without any sign of buckling [20]. Additionally, considering the gravity load, the panel can resist a load demand which is higher than the critical load. It is mentioned that the gravity load would not cause any problem although it is crucial to be considered [20]. The straight shear plates should have sufficient resistant for the lateral horizontal displacements and enough stiffness to avoid excessive sway of the structure [20].

The stiffness requirement in slit shear plates is considered a significant factor of the design criteria, since it controls the design as well as controlling the number of panels required in the frame. It is crucial to consider the rotation of the beams which can have a negative impact on the overall stiffness of slit shear plate frames. The optimum stiffness in which beams can have enough moment of inertia is proposed by Cortes ad Liu [17]. However, generally, the proposed optimum stiffness is approximately $20 \%$ less than the actual real stiffness of the system. Thus, the exact and 
precise equations for the evaluation and estimation of such systems are required. It is noticed that buildings with higher number of stories would experience higher amount of stiffness reduction due to the higher aggregation of rotation. Therefore, comprehensive consideration should be given to the significant factors affecting the stiffness including; the number of panels, the thickness and the location of panels.

It is provided that the width of each link in a shear steel panel can be changed in order to increase the strength and the stiffness of the system. The derived equations for the strength and stiffness of the steel shear plate systems can verify this phenomenon. According to McCloskey [21], the $C / D$ (end-width to thickness) ratio should be in the range of 10-14 to provide enough stiffness against the drifts in higher story levels.

It is mentioned that the use of stiffeners as indicated in Figure 2.5 provides two times more stiffness and a 33\% increase in strength compared to the corresponding steel shear panels without stiffeners [17]. However, the global buckling mode and the stress concentration are associated within this type of structures.
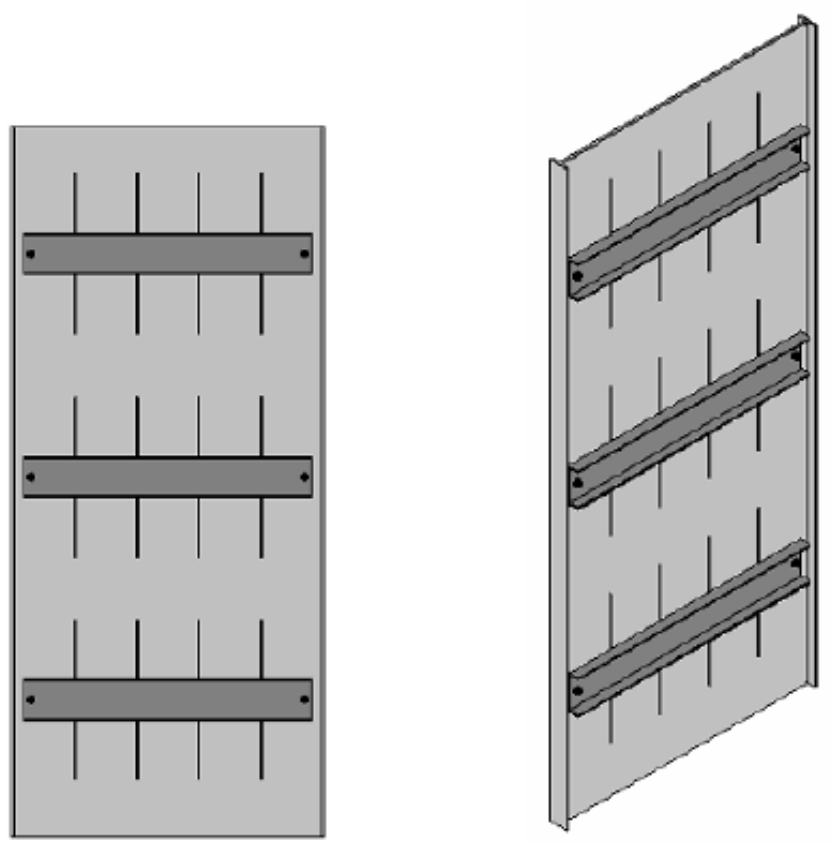

Figure 2. 5 Straight shear links with stiffeners [adopted from [17]] 
In another study conducted by Hitaka and Matsui [22], it has been elaborated that even in detailed designed walls flexural links would experience some inelasticity at the top and bottom parts of the links, in which these regions are the most common areas for flexural limit states. It is provided that the aspect ratio of links undergoing flexural limit state is a significant parameter governing the entire stiffness and strength of the slit walls. Further, the out of plane stiffening is an important parameter in determining the energy dissipation mechanism. Designing such systems in general require a low setting for the first yielding of the damping device in order to trigger the energy dissipation as early as possible, while yielding of the parent structure should be set high enough for the sake of delaying serious damages.

The application of slit panels in various types of structures as structural fuses (replaceable devices) are getting more common. Systems with these damping devices possess two main features which include the stable and large energy dissipation capacity and having a representative model of the cyclic behavior. By arranging these devices in such a way that can be easily accessible, facilitates the replaceability of the devices in the system. Therefore, after every severe event of seismic activity the damaged devices can be replaced with a minimum time and cost, hence interruption to human displacement and occupancy is minimized.

In slit steel shear plates, under the application of small relative lateral loads between the two supported flanges and the upper and lower part of the plate, the strips act as a series of partially fixed-ended beams and deform in double curvature. One important implementation of slit cut steel shear plates is indicated in Figure 2.6 that shows outside of the floor beam area. 


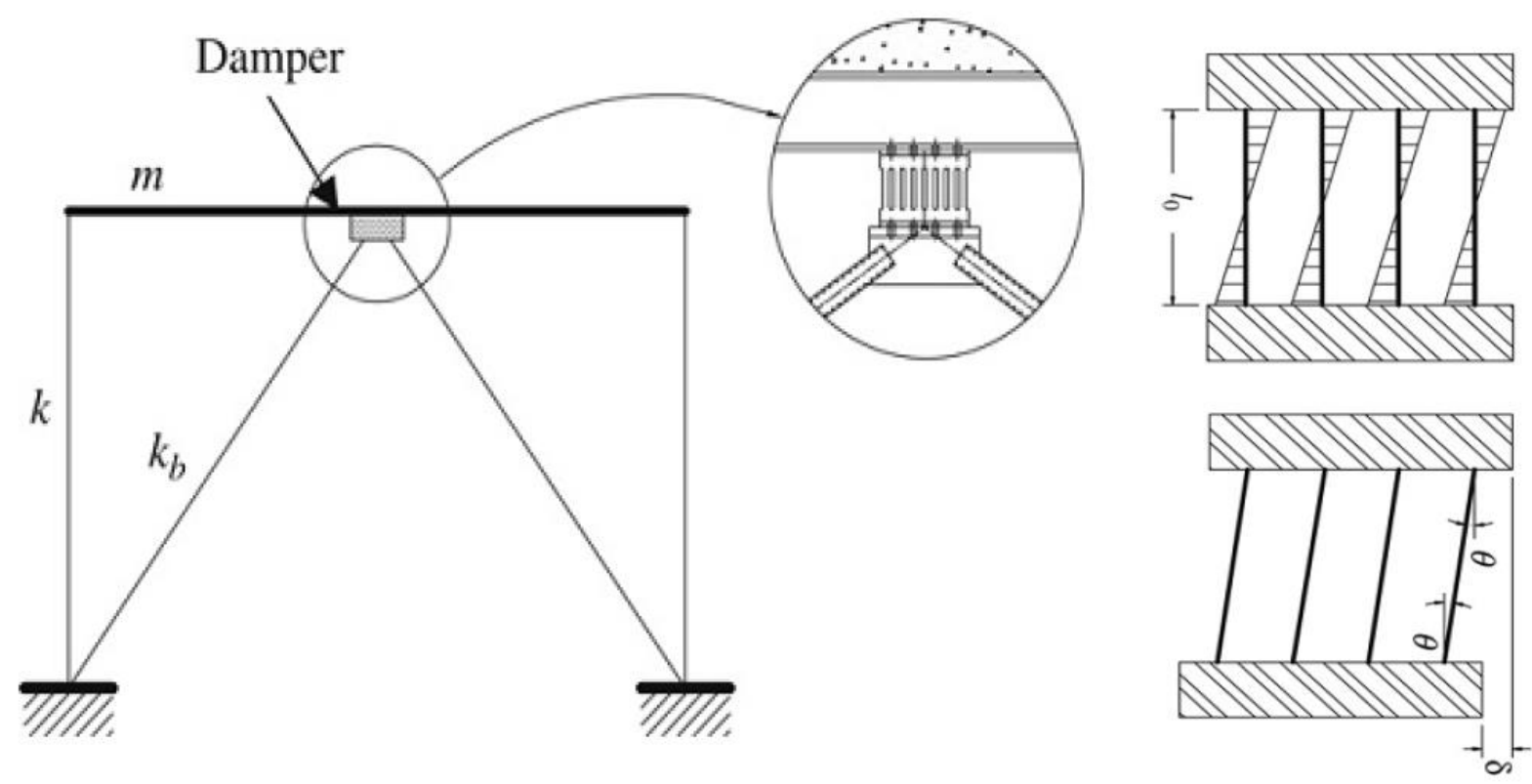

Figure 2. 6 Use of straight shear links in an inverted V braced system [from [22]]

It is observed that the slit links inside of the panel has shown double curvatures. Straight steel shear plates with the highest width to length ratio has shown the best response in terms of carrying more load. The stress concentration is observed at the ends of the links. The accumulation of stress at the ends of the links cause the initiation of cracks and eventually strength degradation. Varying the length of the link has not been found influential in overall behavior of the straight steel shear plates; however, it is resulted in having earlier crack initiation in shorter length links. Lower ratios of width to length in straight panels has shown longer travels and are observed to have a ductility ratio of 29-40 [22].

The lowest slenderness plate has been observed to have a better energy dissipation capacity but has cracked early under small displacement. The equivalent damping ratio and effective stiffness are the two parameters that evaluate the behavior of straight steel shear plates in terms of damping capacity.

Additionally, the ultimate stiffness effects would result in a maximum strength that is as high as twice of the first yield strength [22]. It is mentioned that slit steel shear plates with longer and wider links act more flexible compared to shorter or narrower straight links. However, it is observed that shorter and narrower straight links dissipate more energy compared to wider and 
longer straight links. Moreover, the crack at the ends of the straight steel shear plates' links propagated after 27 cycles of loading which is usually equivalent to $500 \mathrm{~mm}$ of displacement [22].

Along the same lines a new structural system that includes the joint connection of a metallic damper as the beam-to-column connection, is proposed by Oh et al. [23]. It has been indicated that the proposed system has shown significant dissipated amount of energy. Considering the advantages of this newly proposed structural system, the installation of the connection is simple both in construction and in fabrication process. In addition, a superior stiffness is provided within the connection. In contrast, the compression force at the upper part of the steel shear plate can cause local buckling on the beam flange. During any severe ground motion, it is desirable to limit the damage on energy dissipating devices (straight steel shear plates) while the surrounding primary structural members remain undamaged and in their elastic region.

Additionally, according to authors [23], the energy dissipation and plastic deformation have been only concentrated on the straight steel shear plates in this system. The inelastic behavior of the beams and columns is prevented through appropriate design of straight steel shear plates (Figure 2.7).
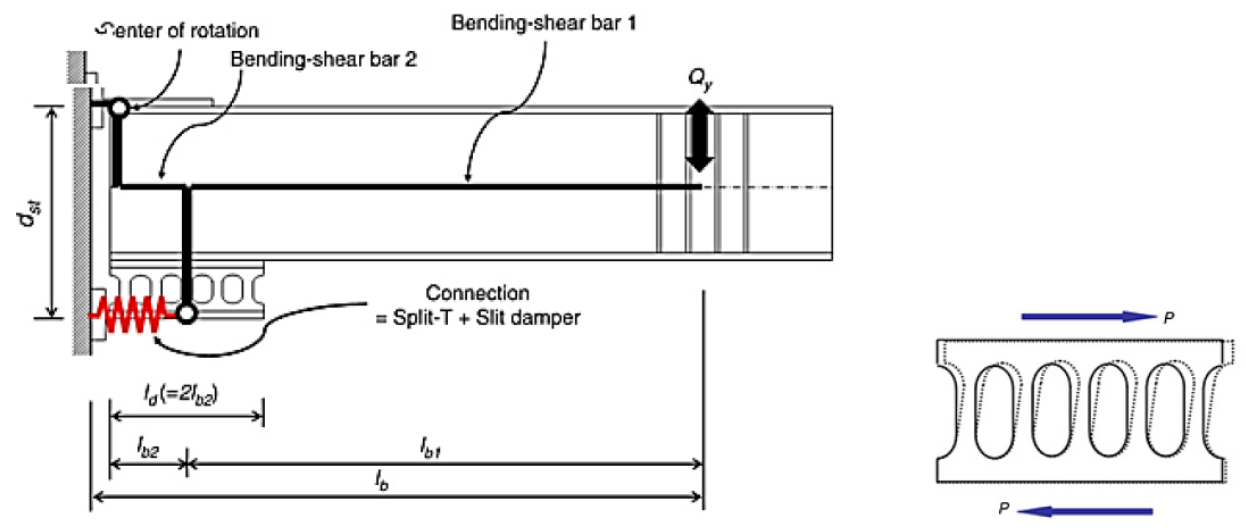

Figure 2. 7 Straight shear links used by Oh et al. [23]

It is also mentioned that for the proposed system, initial stiffness of the system can be controlled by the proper design of the metallic damper (straight steel shear plate) [23]. The initial stiffness of the damper is increased as the strength of the damper is increased. It is stated that, the most wanted feature of the damper is the feasibility in easy installation and replacement of the damper after every severe event of ground motion. It is found that the plastic deformation on the 
system is mainly concentrated at the end of the dampers while the primary structural components, beams and columns, remain almost elastic.

Furthermore, it is stated that with the use of straight links, desirable amount of energy dissipation can be achieved if the width to thickness ratio is kept around 10 [13]. In the straight shear plates, the location of hinge is expected at the ends of the links while for butterfly-shaped steel plates plastic hinges are formed depending on the ratio of mid-width to end-width of the links.

Along the same lines, Koken and Alpaslan Koroglu [24] have worked on the implementation of slit dampers at the beam to column connection zone. The study is concentrated in limiting the damage only to slit dampers under heavy lateral loads and keeping the surrounding beam and column elastic and undamaged. Figure 2.8 shows a typically extended end plate that is equipped with a straight damper at the bottom, a connection with straight damper at the bottom and a stiffener at the end. The use of straight dampers at the connection zones has significantly increased the rigidity of the system while reducing the amount of energy being dissipated through the metallic damper. The implementation of the stiffener would increase the carrying moment capacity by $11 \%$. It is stated that, generally, traditionally extended end plate connection has the capacity of appropriate energy dissipation and plastic deformation, while the repair cost of the beams and columns after every major event of loading is impossible and ineffective due to the formation of local buckling limit state.

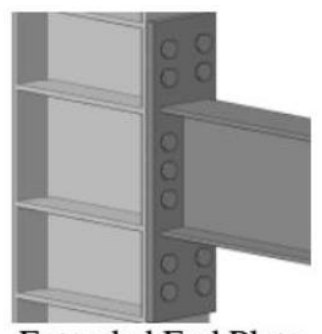

Extended End Plate

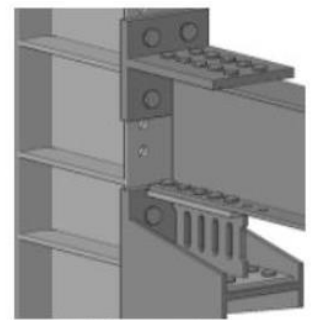

N12 specimen

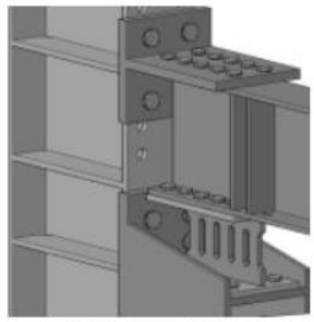

N15 specimen

Figure 2. 8 Use of steel slit shear links in beam to column connections [ from [24]]

Chan and Albermani [20] have done an experimental study on steel slit dampers to investigate the amount of passive energy being dissipated by the device. The device has a basic design of short length wide flange and a web with number of slits in it. Rounded edges at the corner of the slits result in reducing the stress concentration. The device yield load along the stiffness is found based on the plastic mechanism analysis. Cyclic performance and structural characteristics 
are found through the experiments. After the cyclic loading until the specimens' failure the displacements are measured independently by a set of LVDTs.

In the study by Chan and Albermani [20], it is concluded that slit dampers show stable hysteretic behavior and dissipated significant amount of energy. The predicted values for the yield strength by plastic mechanism analysis and the empirically found stiffness were accurately compared with the results obtained from a nonlinear FEM (finite element model), and the predictions are found to be accurate. So, any suitable design can be obtained from the plastic mechanism analysis. Strain hardening effect caused an increased amount of energy dissipation. It is mentioned that longer and wider slits behave more flexibly whereas shorter and narrower slits possess higher stiffness; thus, dissipating energy at higher rates but suffer from early failure. It is observed that large plastic strains concentrations at strip ends caused the specimens to fail by fracture after an average loading of 27 cycles with a cumulative displacement of over $500 \mathrm{~mm}$.

Along the same lines, several studies have been conducted on the designs of steel plates having slits or openings to investigate their energy dissipation capability. In a study which has been reported by Nakamura et al. [25] and Chan and Albermani [20], relatively steel slit dampers which are mostly possessing a link width-to-thickness ratios between 1 and 2 exhibited stable hysteretic behavior up to a peak shear deformation between $10 \%$ and $20 \%$. A fracture is observed in the dampers due to low cycle fatigue having an ultimate strength twice of the yield strength (Figure 2.9).

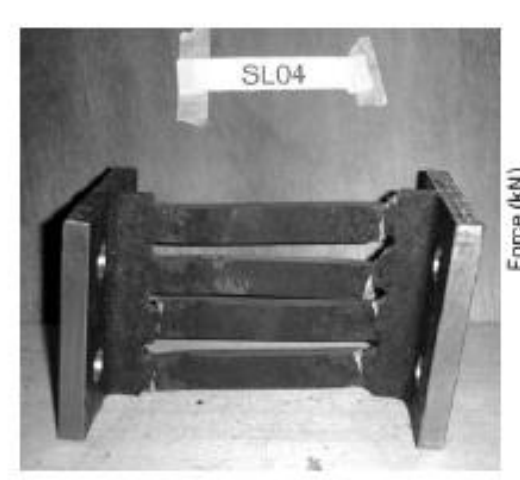

(a)

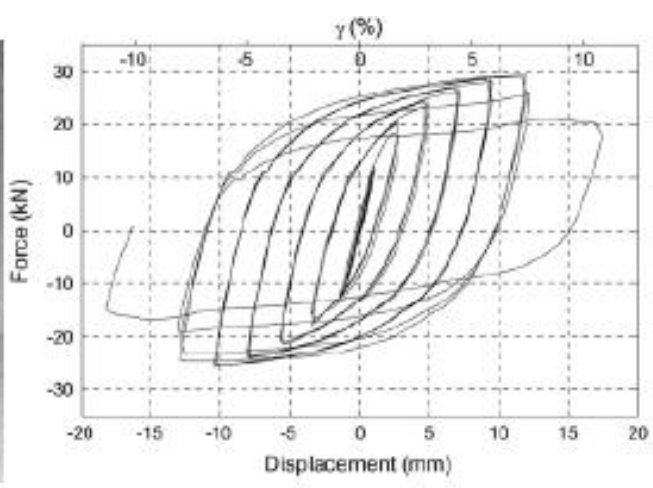

(b)

Figure 2. 9 Test of steel slit dampers: (a) Observance of fracture in the specimen; (b) Hysteresis response [from [20]] 
In another study which has been conducted by Hitaka and Matsui [19], the energy dissipating capacity of thin steel slit walls has been investigated. The walls are used as shear walls in buildings (Figure 2.10) that are designed to occupy a full storey height. Their purpose of utilization makes them much larger and slenderer when compared to the slit dampers, thus having a lower shear drift demand. Limiting the link width-to-thickness ratio to 10, the steel shear wall with slits exhibited approximately $2.5 \%$ of shear drift without any sign of buckling.

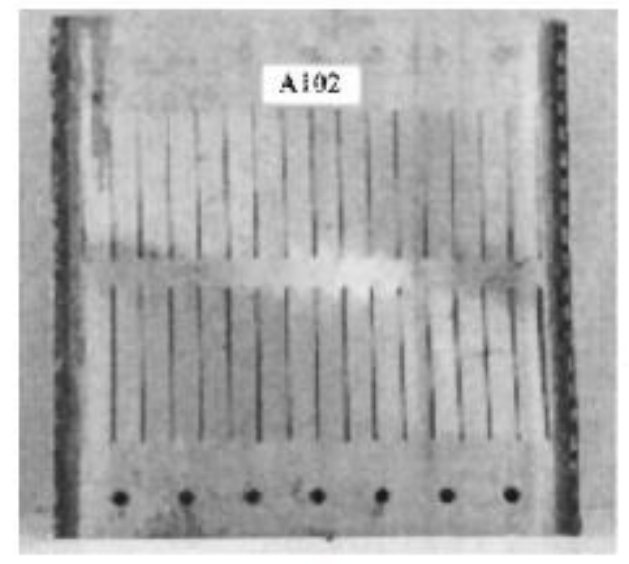

(a)

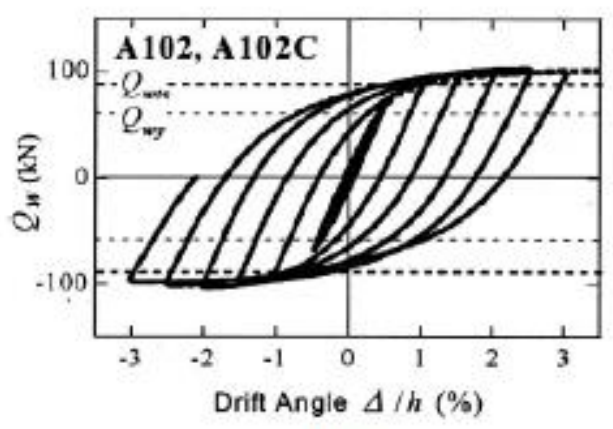

(b)

Figure 2.10 Test of steel shear walls with cuts: (a) Tested Specimen; (b) Hysteresis response [from [19]]

\subsection{Hourglass-shaped Steel Shear Plates}

Hourglass shaped dampers are another type of steel shear plate that are used for energy dissipation. These metallic dampers have circular cuts in the steel plate, to have the occurrence of yielding mechanism before the buckling limit states. Aschheim and Halterman [26] have made a study on the performance of a beam with circular openings in the web. Reducing the web section is another way of designing a web in order to shift the yielding from the connection to the beam and limit the occurrence of the damage in the connection (Figure 2.11). The installation of large circular openings in the web are to prevent the inelastic deformations at the connection area. This study has been conducted based on the recommendations of improving the connections behavior after the Northridge earthquake. Except the links, all the steel has been assumed to have a yielding strength of $345 \mathrm{MPa}$. The yielding for links has been assumed to be $250 \mathrm{MPa}$. 

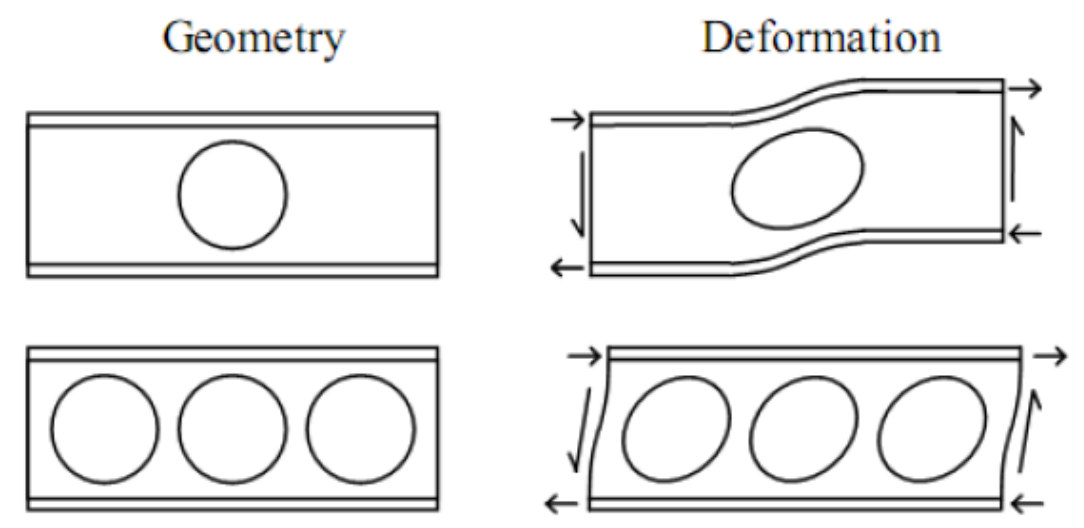

Mode A

Mode B

Figure 2. 11 Shifting the location of hinge away from connection [from [26]]

The gravity loading distribution was a concern for this test. In the test done by Aschheim and Halterman [26], the load has been applied laterally on the specimens. Therefore, when the distributed vertical load is applied on the beam, the shear load at the end of the beam, where location of the openings is, is greater than middle section load. At the ends, neither of flanges nor the reduced web would not be able to resist the vertical shear load.

In the tests done by Aschheim and Halterman [26], the two-sided opening could be an option as far as the energy dissipation capacity is not a matter of concern. It is observed that changing the opening size, would not have the desirable condition as if the flow rate would change and the degradation of strength and stiffness would happen. Delaying the limit states, particularly, the buckling limit state is significantly required. The configuration of specimen 5 (having twosided openings and elliptical openings in the middle part), has shown a better response in terms of stiffness, strength and energy dissipation. Under service loads, the stiffness of the beam is expected to be similar that of an un-perforated beam. Thicker plate is recommended to postpone the occurrence of buckling but, in general, no well-established procedure for the evaluation of the behavior of the beam is presented. It is observed that the connections remain elastic up to a drift ratio of $11 \%$. The yielding in the web of the beam initiates at around $1 \%$ drifts, and buckling would occur at a drift ratio of $1.5 \%$. The significant concern is that after the web is buckled the strength would be significantly decreased - by $21-33 \%$. This percentage is further increased in plates with lower thicknesses. 
A new perforated steel plate shear wall has been designed by Koppal and Eatherton [27] with thicker plates. Early buckling and pinched hysteric behavior due to very thin web plates is often observed in steel plate shear walls. These systems require large-sized beams particularly in the first and last floor to tolerate the diagonal tension field imposed by the plate buckling. Additionally, steel plate shear walls with solid plates need to be supported by moment connections to create resistance against reversed loading condition because after plate is buckled no stiffness can be expected in the plate unless the direction of the post-buckling diagonal tension field is opposite. Therefore, the design of steel plate shear walls with lower thicknesses, and higher sized boundary elements are inefficient.

The proposed shape by the authors (Figure 2.12), has shown less strength compared to the solid steel plate shear wall; however, the early buckling is prevented and the less demand for boundaries is achieved. Moreover, the strength, stress distribution, ductility and energy dissipation capability can be controlled by varying the size and spacing of the openings in the steel plate shear wall. Having butterfly-shaped link in the metallic dampers are observed to be effective in dissipating seismic energy due to the location of plastic hinges formed at the quarter of the links' ends and the yielding distribution over the length of the link.

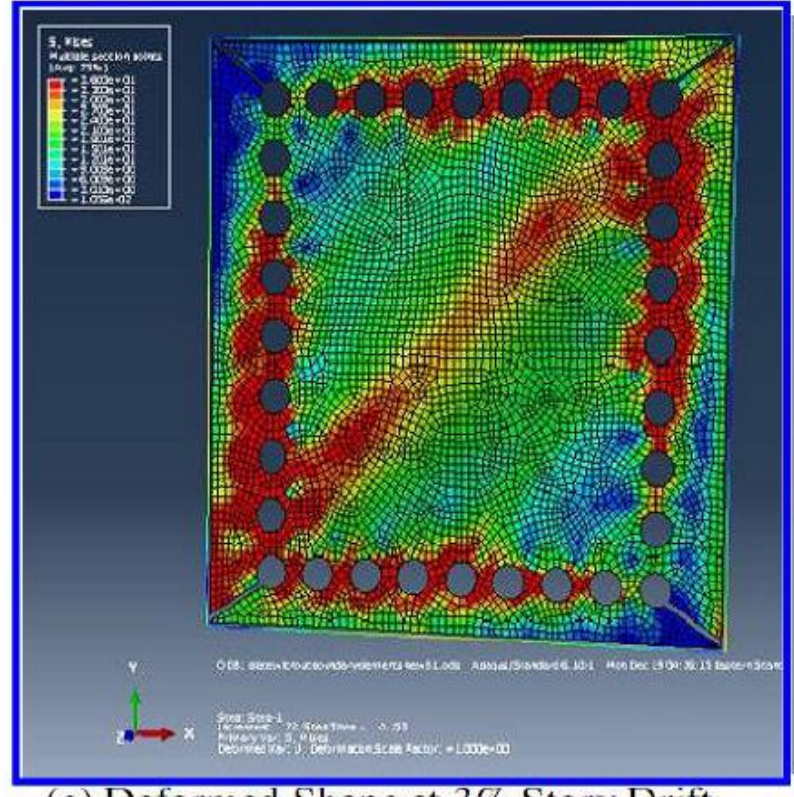

(a) Deformed Shape at 3\% Story Drift

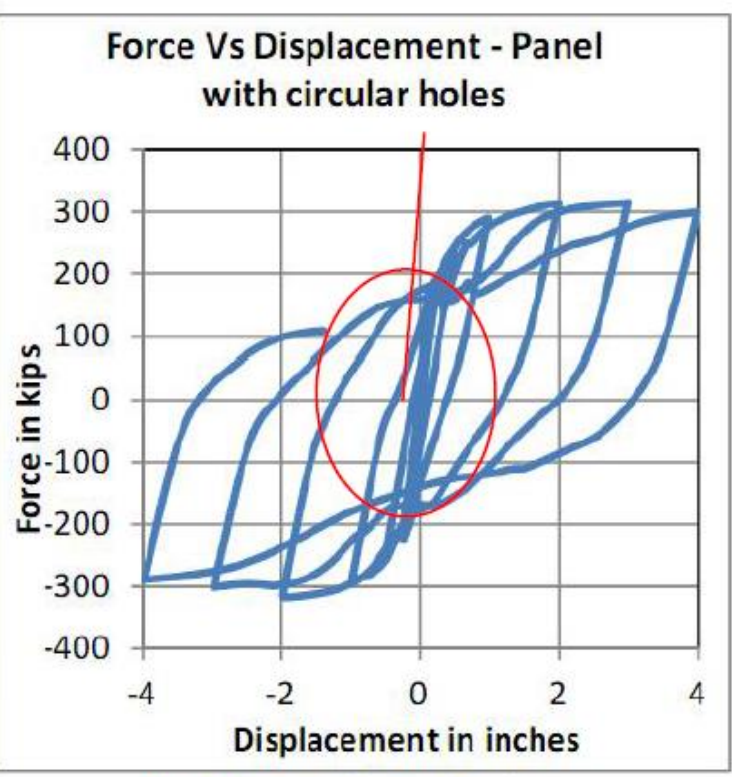

(b) Hysteretic Behavior

Figure 2. 12 Perimeter perforated model proposed by Koppal et al. [from [27]] 
The implementation of thinner web plates is recommended by the designers due to high strength developed by the steel plate shear walls' tension field. As shown in Figure 2.12, the initial stiffness of the plate is kept in the desirable range and the pinching behavior of the plate is eliminated. Additionally, providing straight openings on the corner of the shear wall significantly reduce the stress concentration resulting in fewer demands on the connections which results in a reduction in less boundary elements inward acting loads.

It has been suggested that the use of butterfly-shaped perforation could result in larger strength compared to the implementation of circular perforation. Further, in perimeter perforated steel plate shear walls, reducing the spacing would result in decreasing the strength and the stiffness. The circular perforation spacing has a significant impact on the response characteristics and increase in thickness. In perimeter perforated steel plate shear walls increasing the thickness would result in higher energy dissipation capability.

Along the same lines, Kawai et al. [28] have worked on the steel plates with circular openings inside. It is observed that, the presence of ring-shaped ribs of circular holes significantly prevented the occurrence of large out-of-plane waveforms. Steel plate shear walls with circular holes have shown large initial stiffness, stable strength, and constant energy dissipation (Figure 2.13).
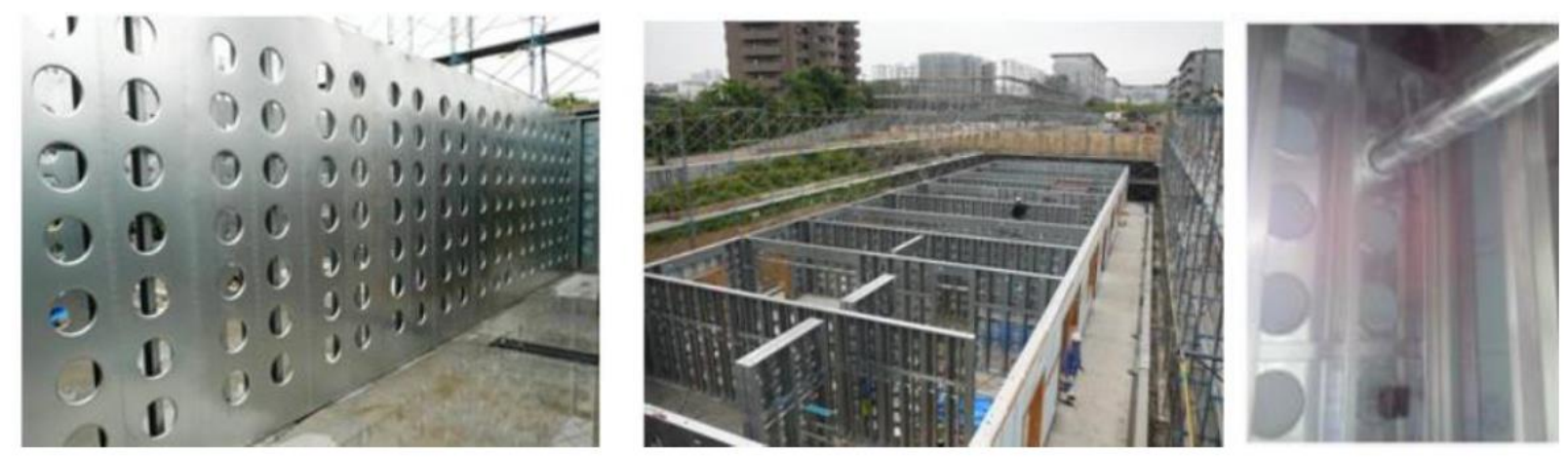

Figure 2. 13 Steel sheet wall with circular holes [adopted from [28]]

It is observed that under medium to large seismic forces, the stress concentration has occurred at the intervals between the aligned burring holes, and the system has experienced elastic behavior with no evidence of damages. The concentrated stress eventually reaches its ultimate condition due to shear buckling or yielding. It is stated that, the performance of the steel plate shear 
wall is improved by having ribs around the circular holes. As shown in Figure 2.14 the ribs around circular holes prevent the out-of-plane waveform. It is worth to mention that, the plate thickness was $1.2 \mathrm{~mm}$, which causes the early buckling limit state. It is concluded that steel plate shear walls having bearing holes concentrated the stress between the aligned holes which ultimately govern the shear buckling and shear yielding.

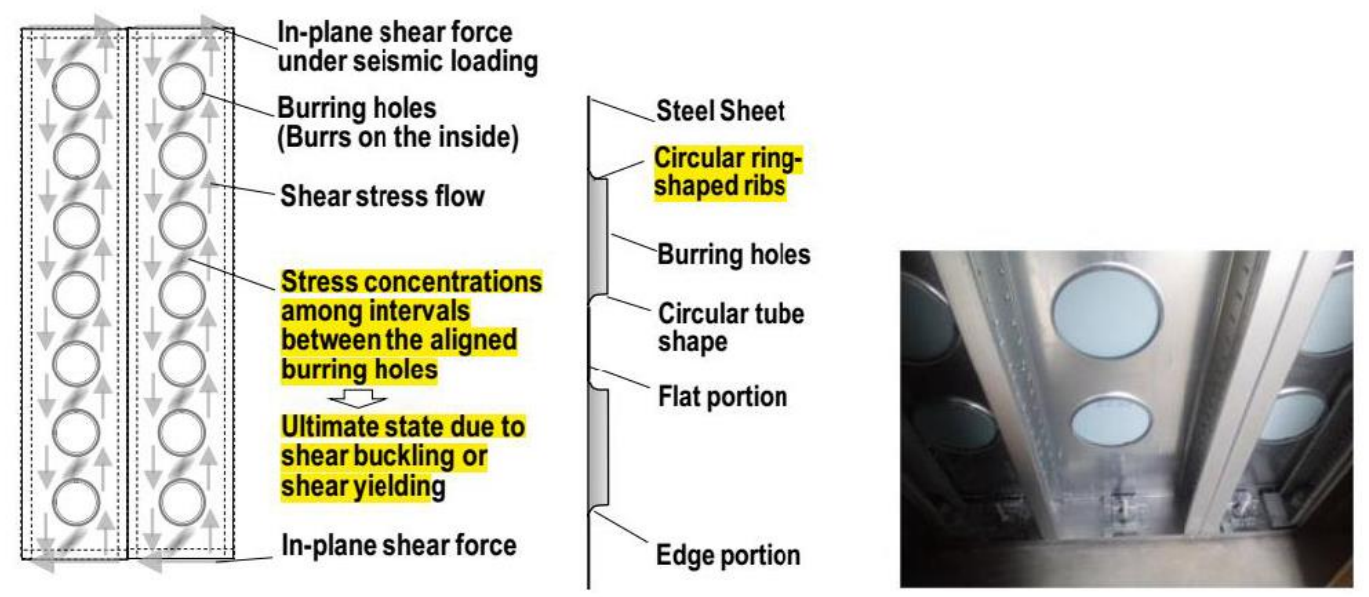

Figure 2. 14 Use of ribs on the Circular holes [from [28]]

\subsection{Triangularly Shaped Steel Shear Plates}

Asselin et al. [29] have investigated the effect of use of triangular steel plates in hybrid systems. The system is constructed of a masonry wall having triangular steel plates inside. The triangular steel plates are connected to a steel frame (Figure 2.15). The steel plate has been fabricated from a 6 -inch $(=152.4 \mathrm{~mm})$ wide by 0.5 -inch $(=12.7 \mathrm{~mm})$ thickness A36 flat bar using a plasma cutter. In general, there are two design approaches for these systems, first, the steel plates are designed to be strong enough to remain elastic during the loading and damage is concentrated in the masonry wall. The second approach considers the design of steel plates to act as an energydissipating element, thus allowing the masonry wall to remain undamaged. The second approach have been used and investigated in this research. 

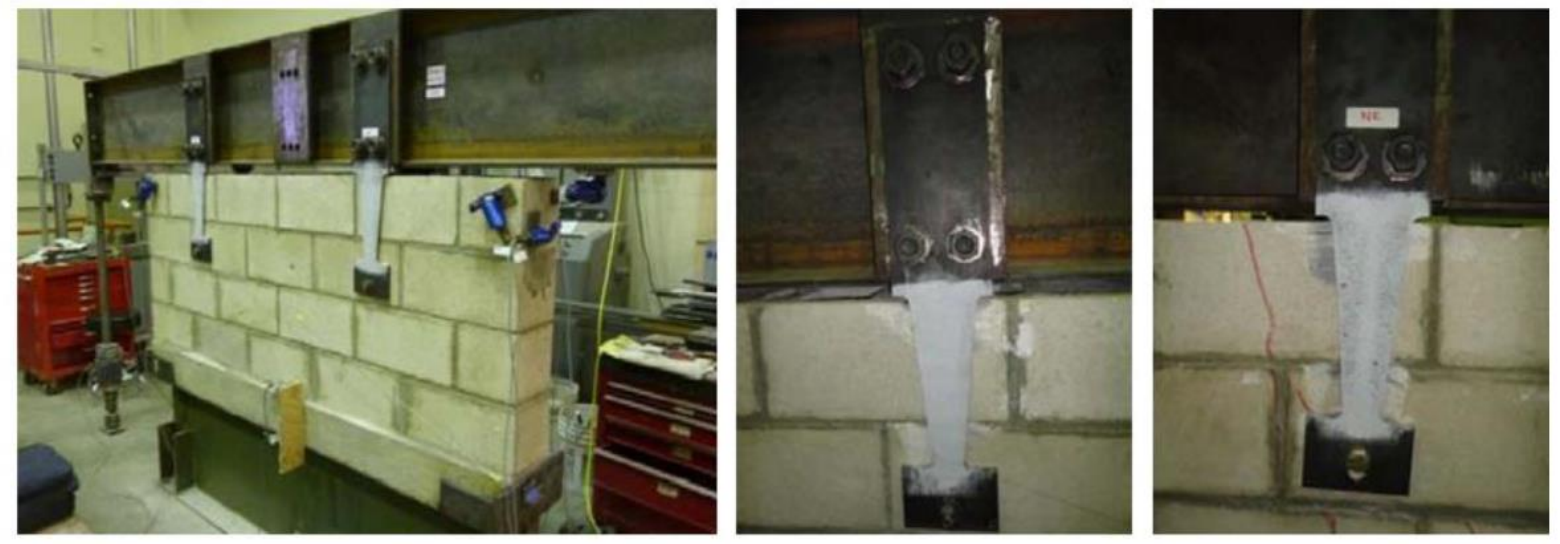

Figure 2. 15 Implementation of triangular shaped shear links in masonry structures [from [29]]

A maximum lateral load of $20 \mathrm{kips}(=88.95 \mathrm{kN})$ and maximum displacement of 2.75 inches $(=69.85 \mathrm{~mm})$ has been observed. The triangular steel plates which have been the source of energydissipation have lost a significant amount of stiffness and strength due to the torsional buckling. It is noticed that the torsional buckling of the steel plate should be investigated thoroughly to avoid the immediate strength and stiffness degradation in the triangular steel plates. After having different story level buildings, the use of this kind of structures with lower heights and in places of higher seismicity is concluded.

\subsection{Ring-shaped Steel Shear Plates}

Numerical evaluation of ductility and energy dissipation of steel rings have been investigated by Andalib et al. [30]. Specific materials are defined for bolts that are connecting the plates. Steel rings are merged into connecting plates and are bolted with the fixed boundary plate (one model is semi welded one model is bolted). One side is fixed while the other side is left under the application of an axial force. Three different models with various connections are simulated in ANSYS to find out the effects of steel ring connection on their performance. Influence of material properties and the thickness of half rings is also investigated. A cyclic loading is applied, and displacement control loading is calculated based on the yielding displacement of the model. Based on the load displacement result of the simulated ring under the cyclic loading, two linearization have been performed by FEMA 356 proposed method. The maximum vertical displacement and 
corresponding vertical displacement of the system the end of the tensile elastic limit is found then the ductility of the model for both the tension and compression is calculated.

After considering a comparison between the energy dissipation capacities of different model's of steel rings throughout the study by Andalib et al. [30], the following major factors are concluded: Steel rings which are made of two half rings have successfully dissipated energy through inelastic behavior of the rings and are substantially ductile; use of different materials significantly change the amount of energy dissipation and the ductility; and use of different thicknesses change the amount of energy dissipation and the ductility in steel rings.

\subsection{Honeycomb-shaped (butterfly-shaped) Steel Shear Plates}

The shape of slit steel plates has been altered by Kobori et al. [31]. Honeycomb-shaped openings are cut instead of previously used rectangular openings, which left butterfly-shaped links (Figure 2.16). The resemblance of butterfly-shaped links to the moment diagram of beams deforming in double curvature resulted the plates to have a more distributed yielding along the length of the link. The butterfly-shaped steel plates have been made thick enough, with a widthto-thickness ratio of 2.5 and length-to-thickness ratio of 6.7 , to avoid buckling. Based on the performed test results, the dampers have exhibited $30 \%$ of shear deformation without stiffness degradation.

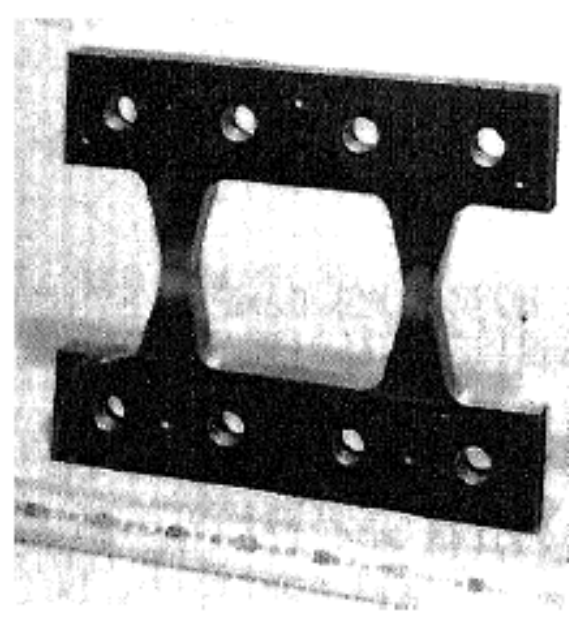

(a)

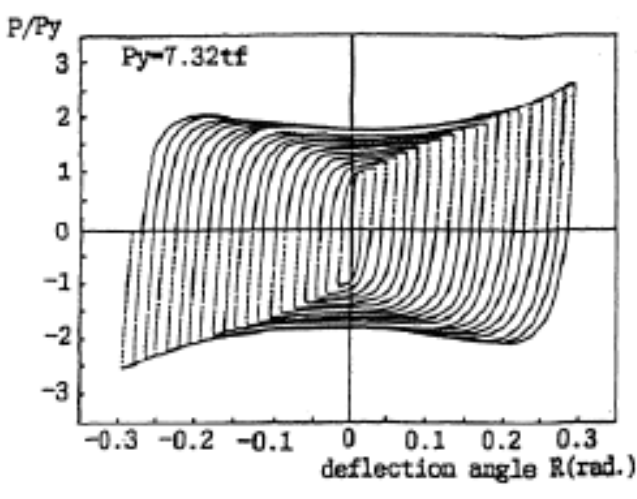

(b)

Figure 2. 16 Experimental test of Honeycomb dampers: (a) Tested specimen, (b) Hysteresis response [from [31]] 
In the study which has been made by Kobori et al. [31] the goal was to decrease the response of high-rise structures through two advanced damper arrangements as shown in Figure 2.17. The purpose of using butterfly-shaped dampers in the structural framing systems is to obtain high stiffness and less storey drifts. Joint dampers which are solidly bolted to every story at the upper and lower extended plates, are allowing the middle section to horizontally drift with the plastic range of the damper. Joint dampers are used to connect any two adjacent structural elements under different types of loading vulnerable to any direction. The middle section shape is designed to achieve the purpose of uniformly distributed yielding over the length of the damper. The difference in between the joint damper and the Butterfly-shaped damper is the load demand. Butterfly-shaped damper is designed to be used when the load is acting in the plane of the damper while joint damper can be used with any loading in any direction. However, the limitation of movement in the joint damper makes it less efficient in dissipating energy. Having said that, the hysteretic behavior of both dampers makes them suitable in areas with high seismicity due to low amount of pinching observed in the dampers.

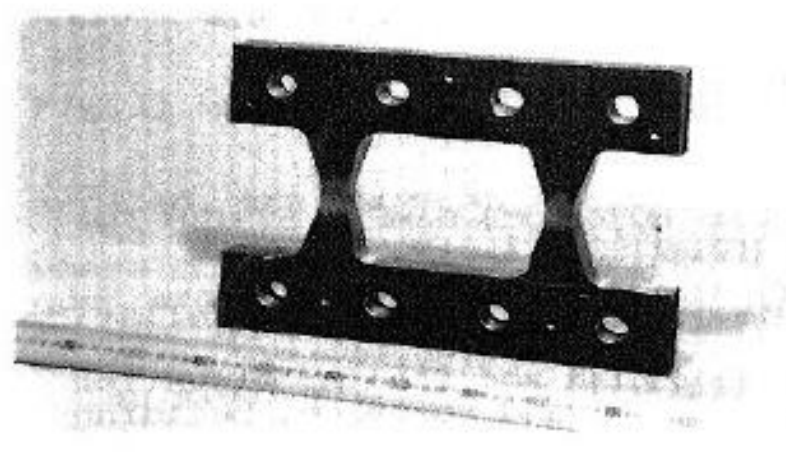

a) The Butterfly-shaped damper

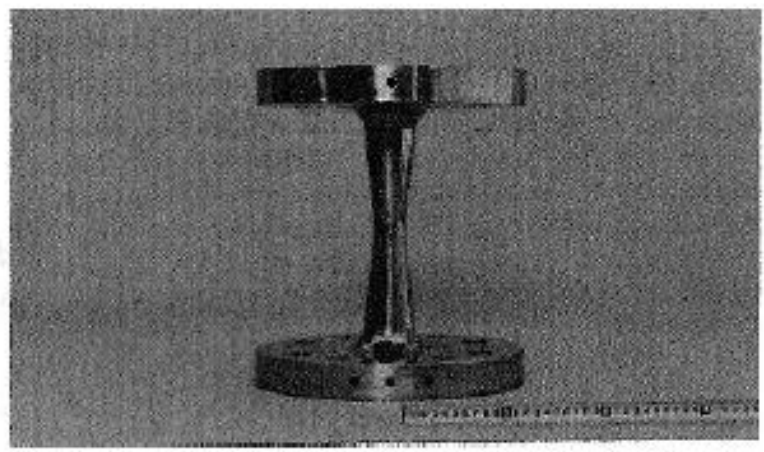

b) The joint damper

Figure 2. 17 Hysteretic Dampers [from [31]]

Several examples of buildings show the utilization of the notion of butterfly-shaped dampers. In the example shown in Figure 2.18, butterfly-shaped fuses are rigidly fastened with high strength bolts in between the detached walls connecting lower and upper beams of the storey. In this way, the storey movement is concentrated in the butterfly-shaped fuses. 


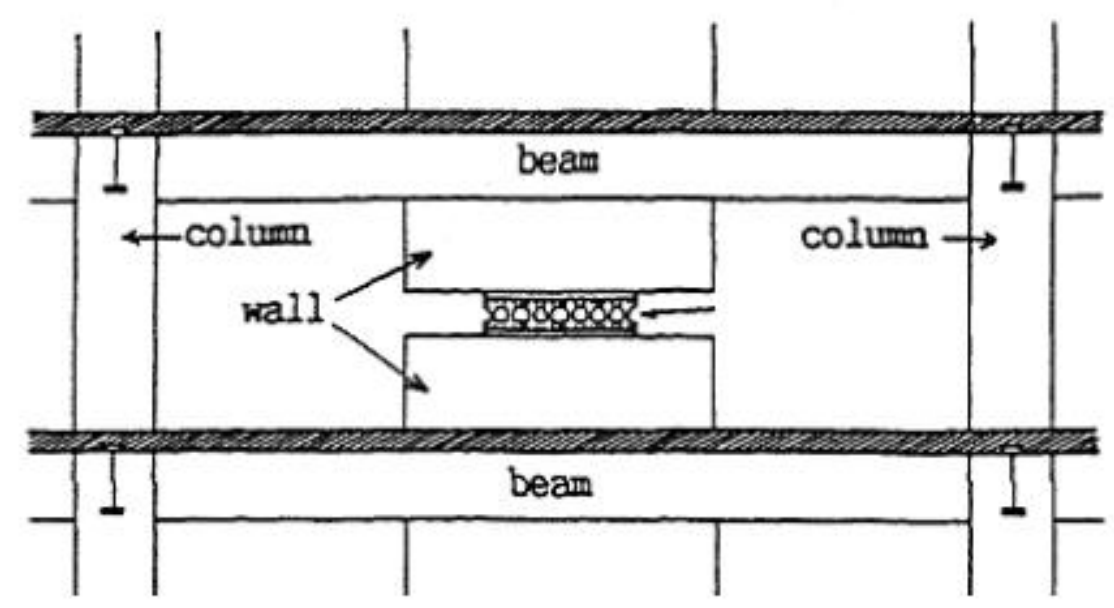

Figure 2. 18 Utilization of Butterfly-shaped links in wall systems [from [31]]

In the above example, the effectiveness of the amount energy dissipation of the butterflyshaped fuses is investigated. A damper with a height of $135 \mathrm{~mm}$ is designed to yield against the bending stress caused by the lateral displacements. Pushover curves are used to observe the amount of energy dissipation. Walls using butterfly-shaped dampers in their detachment sections with the upper and lower beams have shown a reduction of $65 \%-90 \%$ and $55 \%-122 \%$ for the maximum storey shear and maximum storey angles, respectively, when compared to walls where no detachment is existed. This proves the significance of butterfly-shaped fuses in the amount of energy dissipation.

Hanson et al. [32] introduced another example of the usage of butterfly-shaped fuses in the structural framings. The butterfly-shaped fuse is used with a set of damping devices named as added damper and stiffness in structural passive control systems (ADAS Figure 2.19). As the stress distribution of the butterfly-shaped fuses caused by the flexure is uniform along the length of the fuse, it makes it desirable as an out-of-plane implementation. When the ADAS is horizontally moved relative to the bottom it will dissipate energy. 


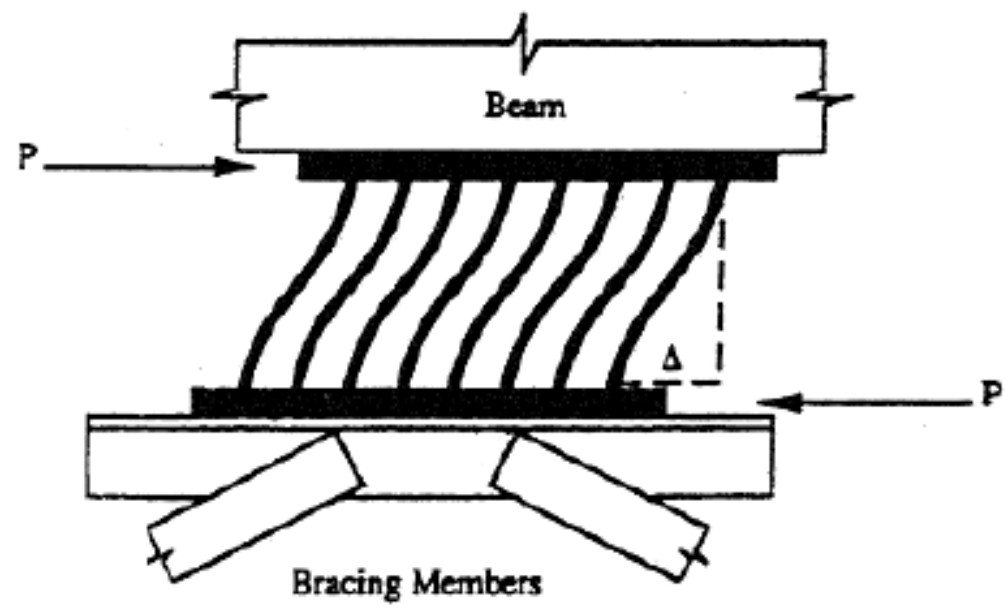

Figure 2. 19 Added damper and stiffness (ADAS) Concept [from [32]]

An experimental study has been made to investigate the effectiveness of the yielding elements by constructing two frames with and without the ADAS system. Based on the evaluation of the experimental results, the use of ADAS system has induced more stiffness and strength as well as having a more stable energy dissipating behavior (shown in Figure 2.20). Hanson et al. [32] has conducted a parametric research which resulted in stating that the better behavior is provided by the lower material yield displacement, yet to control the ductility of the device the yield displacement should be sufficiently large. Experimental studies by Hanson et al. [32] have stated that using of hysteric dampers would increase the strength, stiffness as well as the energy dissipating ability of the entire system even if they are bent over their weak axis. It is observed that these devices preserve a substantial cycles of load reversals as high as 100 . Thus, it is recommended to give substantial significance to the yielding displacement to have specific values for ductility. The yielding displacement is said to be 0.0014 to 0.002 times of the story height. 


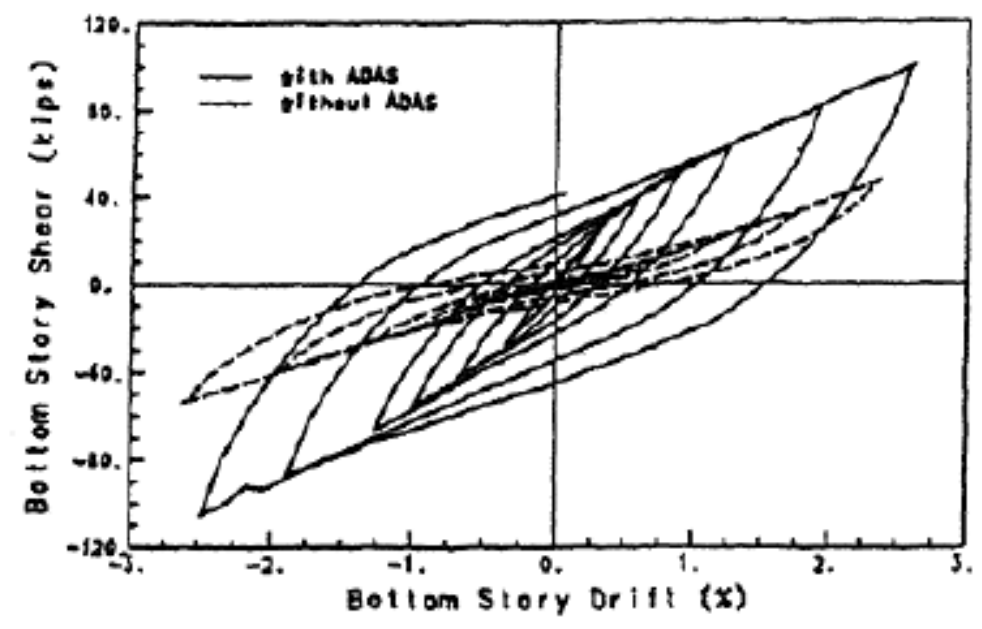

Figure 2. 20 The effect of ADAS on the hysteresis response of the frame [from [32]]

Further studies have been made by Koppal and Eatherton [27] to have the shear or flexural yielding domination instead of buckling. They have used butterfly-shaped links in steel plates. It is claimed that, increasing the thickness of the plate will result in having a higher ultimate strength. Additionally, a change in thickness is considered to be more effective in developing the stiffness rather than strength. By their study, it is recommended to have a plate thickness of more than 0.5 inches $(=12.7 \mathrm{~mm})$ to have an appropriate amount of energy dissipation. This value for the thickness is considered high when it is compared to other studies.

A recent study which has been made by Koborie et al. [33], the use butterfly-shaped fuses is investigated in high-rise and partially mid-rise structures against earthquake loadings. It is found that with the use of butterfly-shaped fuses the structure will shake less when it is imposed to seismic motions. It is also stated that butterfly-shaped fuses will reduce the response of the structure by $20-30 \%$ without having the damper destroyed after every single major event due to the margin rotation ability of $0.5-0.25$ degrees.

\subsubsection{Low Cycle Fatigue Test on Butterfly-shaped Steel Shear Plates}

Further studies have been conducted by Tagami et al. [34] to investigate the fatigue behavior of honeycomb dampers. Manson-Coffin equations have been used to characterize the honeycomb dampers. The bending capacity and moment distribution of honeycomb-shaped 
dampers has reached to a better alignment using ADAS device. Dynamic tests have been conducted on ADAS device to check the safety purposes. The tests showed that ADAS devices can be safely designed to undergo deformation ranges up to 10 times of the yield deformation (Figure 2.21).

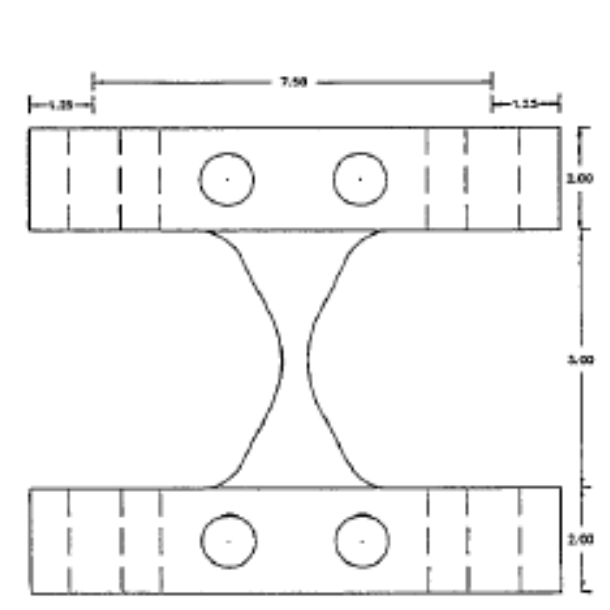

All dimensions in inches

(a)
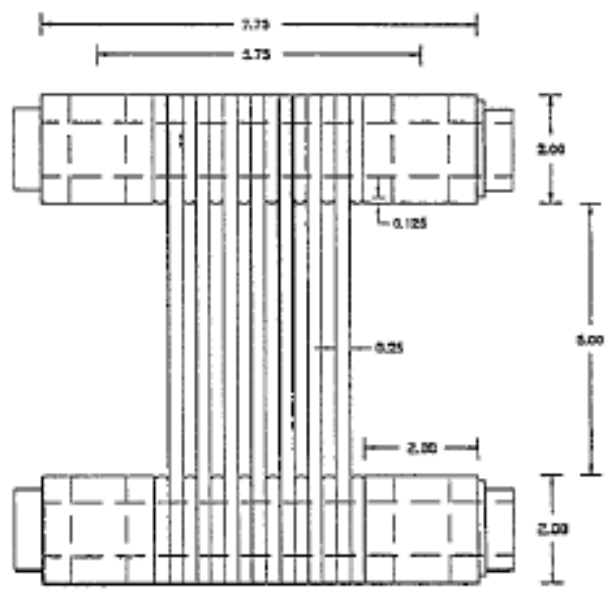

All dimensions in inches

(b)

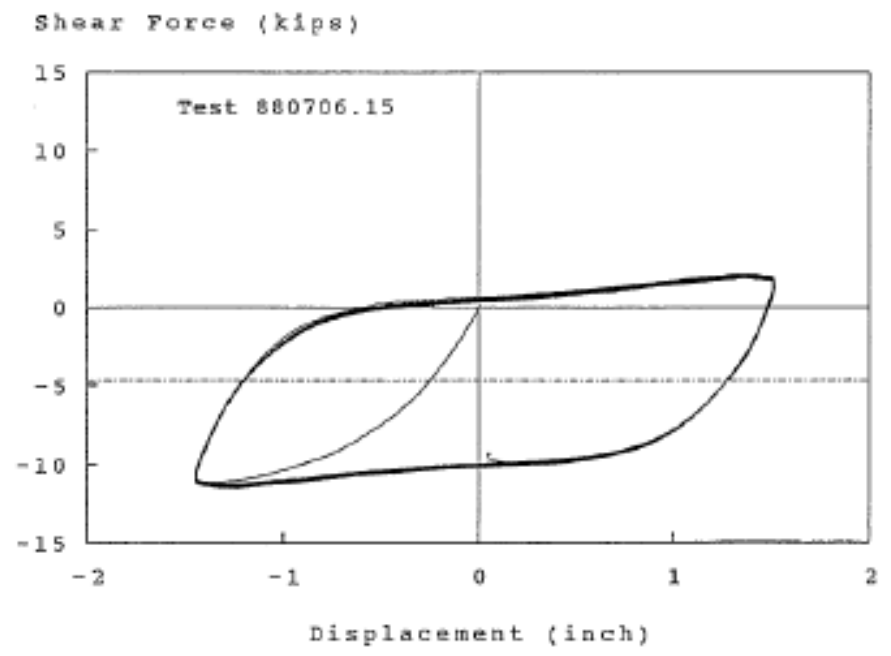

(c)

Figure 2. 21 Experimental Tests of ADAS elements: (a) specimen's section perpendicular to the direction of loading; (b) Specimen's section parallel to the direction of loading; (c) Hysteresis response [from [34]] 


\subsubsection{Cyclic Performance of Non-uniform Butterfly-shaped Steel Shear Plates}

Lee et al. [15], have conducted an experimental study on three various shapes of nonuniform strip dampers to have a deeper understanding on the behavior of the butterfly-shaped links. Straight dampers are also studied in parallel to butterfly-shaped links with uniform and nonuniform thicknesses. The experimental results have shown that the butterfly-shaped links have had a better hysteric behavior under cyclic loading when compared to straight dampers. The straight dampers are made to function in low strengths. The geometry of the straight damper enables the damage to be concentrated at the two ends of it, thus, ultimately, decreasing the amount of energy being dissipated in the system when compared to butterfly-shaped fuses. It is recommended that high ductile steel dampers which has a lower yielding point should be used in the structure that will have more control on the vibration. In the tests performed by Lee et al. [15], the specimen's thicknesses varied between $10 \mathrm{~mm}$ to $17 \mathrm{~mm}$. the reason behind using a thick steel plate was to provide enough resistance against buckling.

Pre-provided stiffness equations by Lee et al. [15], have had to be revised for a new value of $\mathrm{C}$ factor that will take the rigidity of the dampers into account. A C factor of range 0.55 to 0.706 is considered for the stiffness equations of pure-flexural dominated dampers. When comparing the crack distribution, it is observed that the linearly decreased thickness butterfly-shaped fuses have a uniform crack distribution comparing to the other specimens. This is also compared to the straight dampers which will eventually have their fracture at the two ends of the link. Due to an unbalanced distribution of stresses the necking is also observed. Furthermore, it is seen that the better the outof-plane buckling capacity the better the energy dissipation ability. The authors have also studied the damping coefficient for the dampers which had a range of 0.48 to 0.51 . it is also provided that the use of butterfly-shaped links with uniform or non-uniform thicknesses will result in having a higher ductility capacity as well as higher energy dissipation capability compared to straight dampers. The ductility and energy dissipation amount are found to be 1.13-1.75 and 2-2.36, respectively, for butterfly-shaped dampers.

In 2016, Lee et al. [6] have studied the behavior of butterfly-shaped fuses through a combination of non-uniform thickness butterfly-shaped fuses and shear-type friction dampers. The idea behind this combination have been the consideration of the friction damper resisting the load under less-tense cyclic loadings and when the loading is increased the butterfly-shaped fuses 
would dissipate the further energy through the inelastic deformation. According to the results obtained, these dampers have shown significant load-resisting capacity under monotonic load as well as having an excellent ductility and energy dissipation ability. It is also observed that these dampers, strip dampers, have uniformly distributed the damage along the length of the link. When using metallic dampers for seismic purposes, it is important to have the damper providing adequate stiffness to the system under service load as well as the seismic energy should be only dissipated through the dampers not the other surrounding structural components. Therefore, the damper should yield when it is imposed to low displacements. It is also considered to have the stress

concentration away from the middle portion of the link. It also stated that further attention should be made while defining the damper's thickness. The damper should be thick enough to provide sufficient resistance against buckling.

Based on the results obtained from the tests by Lee et al. [6], it is observed that specimens would undergo an axial tension force, double curvature bending and a constant shear force. The dampers which have been tested under monotonic load rested a load of 2 times greater when compared to cyclically loaded dampers. Higher values of the ultimate strength for all the specimens are observed due to the strain hardening. Monotonically loaded dampers had values as high as three times of the yielding values, while the specimens under cyclic loading exhibited an ultimate strength of as high as 1.8 times of the yielding.

\subsubsection{Shape Investigation on Butterfly-shaped Steel Shear Plates}

In another study which have been made by $\mathrm{Li}$ and $\mathrm{Li}$ et al. [35], a new idea of designing metallic damper is introduced. By applying different types of loadings such as quasi-static and ground motions, Li and Li et al. [35] have studied butterfly-shaped fuses under specific shapes shown in Figure 2.22. It is concluded that these fuses have the capacity of dissipating energy up to stop point, which is shown in Figure 2.23. The exhibition of any fracture on the fuses or degradation in the strength by $25 \%$ is defined as the criteria for a stop. 


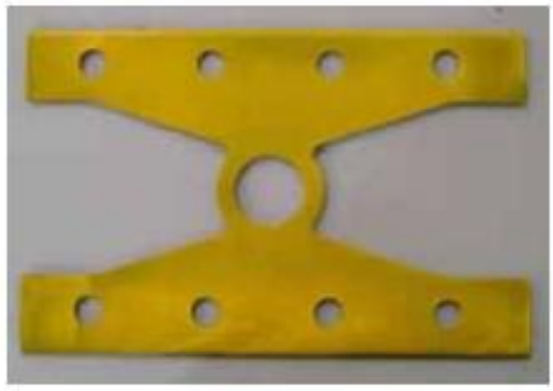

a) Round hole Butterfly-shaped specimen

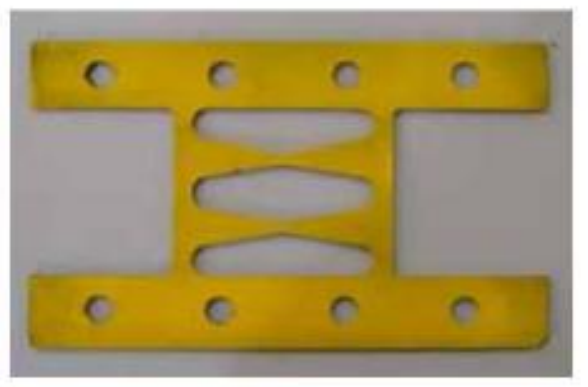

b) Butterfly-shaped damper

Figure 2. 22 Different shapes investigated in the study of Li and Li et al. [35]

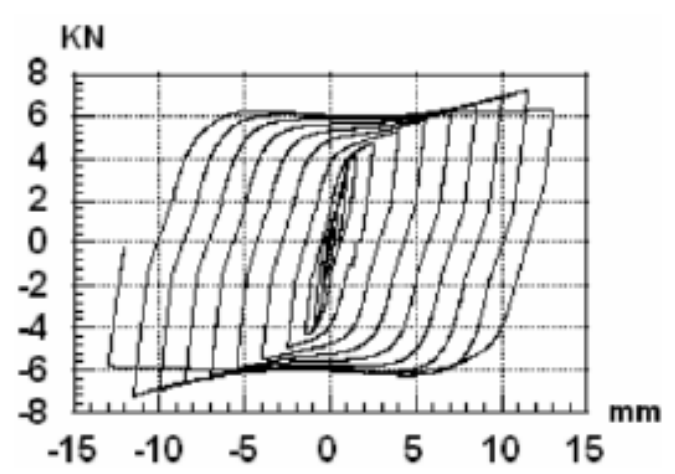

b) Round hole Butterfly-shaped specimen

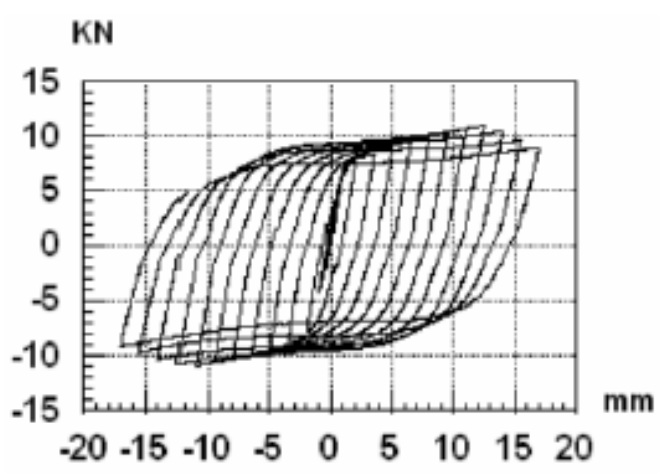

b) Butterfly-shaped damper

Figure 2. 23 Hysteresis response of Butterfly-shaped specimens [from [35]]

It is stated that butterfly-shaped links can provide substantial stiffness as well as enough energy dissipation, as high as $50 \%$ to $90 \%$ of the total energy, when implemented in normal applications. Having said that, the implementation of these devices in real world applications exhibit substantial stiffness and energy dissipation [36].

It is stated by $\mathrm{Li}$ and $\mathrm{Li} \mathrm{[37]} \mathrm{that} \mathrm{supplying} \mathrm{additional} \mathrm{damping} \mathrm{for} \mathrm{structural} \mathrm{systems} \mathrm{is}$ relied on the out-of-plane buckling of the damper. Even the reduction of the response of the structural system under the application of environmental demands is dependent on the out-of-plane bending deformation. Uniform distribution along the length of the fuse is considered advantageous, while due to low out-of-plane stiffness, even if the fuses are subjected to small 
loading, it will undergo inelastic deformations. The inelastic deformations will make the fuse to be replaced after any minor disruption. Through the study, the authors investigated specimens of single round-hole and $\mathrm{X}$ shape dampers. The out-of-plane buckling is observed in the strip region which has resulted a substantial amount of reduction in strength. Use of these devices has shown the peak drift of the system is decreased by four times in different stories when compared to conventional structural systems. However, an increase in the acceleration and base shear is noticed. It is recommended that links having a width to length ratio of one or more are less vulnerable to the out-of-plane buckling.

Based on a previous study of distinct shapes of dampers constructed from mild steel such as concave, convex and straight lines (Figure 2.24), Teruna et al. [14] investigated the hysteretic behavior of four different butterfly-shaped specimens through experimental tests. The four specimens possessed various geometry properties. Results have concluded that all the specimens have close hysteric behavior. Having said that, a more stable and perfect hysteric behavior as well as ductility factor is observed in the convex-shaped specimen. After the comparison have been made between the butterfly-shaped fuses and other types, it is claimed that yielding happens consistently and uniformly along the length of the dampers which would lead the structure to obtain large deflections without any early fracture or buckling. In contrast to butterfly-shaped fuses straight slit fuses would centralize the yielding inelasticity at both ends under the combination of shear and flexural stresses. Furthermore, butterfly-shaped fuses are bolted in an in-plane format which would have its strong axis as the bending axis. This will result in obtaining a higher initial stiffness compared to other traditionally used dampers such as rhombic dampers (shown in Figure 2.25). 


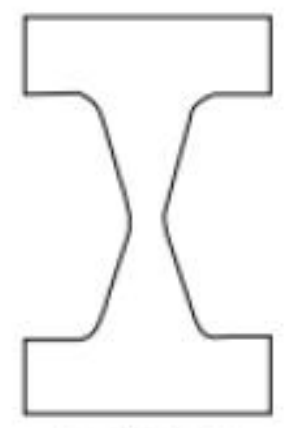

(a) X-ADAS

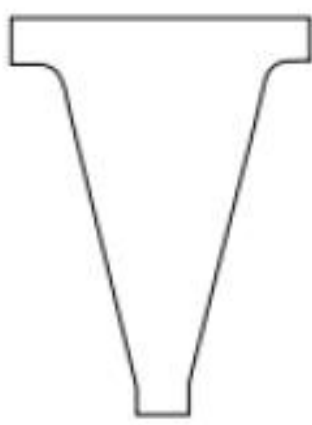

(b) TADAS

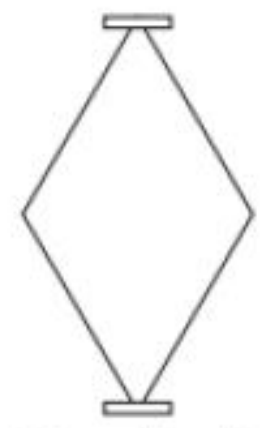

(c) Rhombic damper

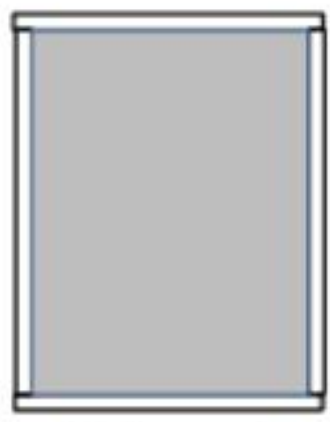

(d) Shear panel

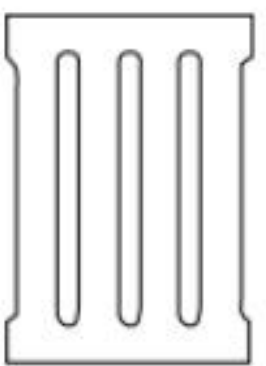

(e) Slit damper

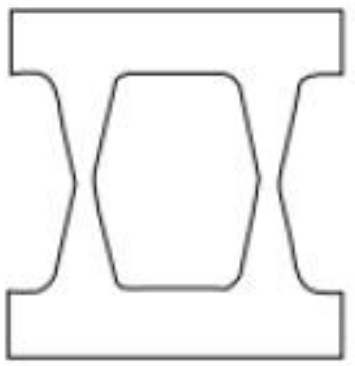

(f) Honeycomb damper

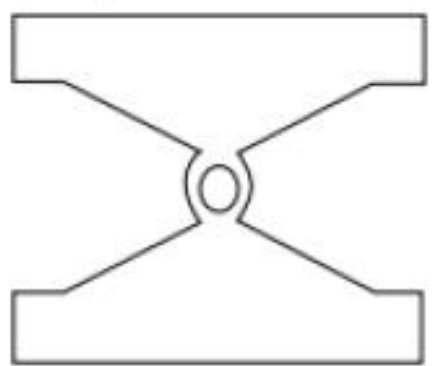

(g) DFMD damper

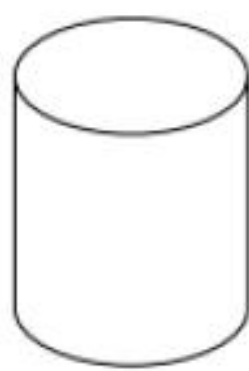

(h) CPD damper

Figure 2. 24 Different purposed dampers [from [14]]
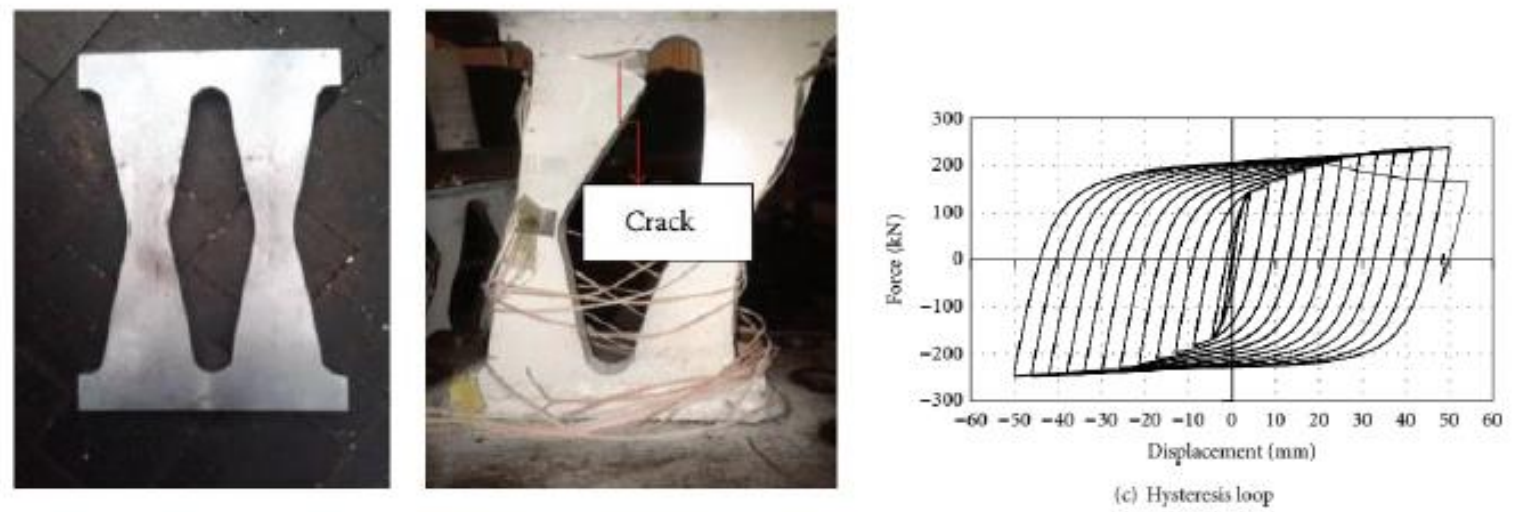

Figure 2. 25 One of the four tested specimens [from [14]]

In the study of Teruna et al. [14] the pushover curves during the loading and unloading have been studied. It is concluded that the maximum strength Vmax, (during the loading) or $V m i n$ (during unloading) is 1.33 and 1.92 times of yield strength respectively due to the strain hardening. It is also observed the specimens would not exhibit the same response under the cyclic loading through the effect of Bauschinger; however, this change will not affect the strength by 
more than 5\%. Also, the effects of various sizes for the fillet at the edges is studied to determine the importance of the stress concentration over those areas. Based on the comparisons of the specimens, the studies have shown that the changes in sizes of the fillet area including the radius would not a make a substantial difference in the strength. Figure 2.26 shows one of the four butterfly-shaped specimens tested by Teruna et al. [14]. The authors recommended further study on one of the tested specimens without considering the reasons behind a better performance of the fuses. Furthermore, it has been indicated that further studies should be to investigate the flexural hinge locations as this fuse has more flexural dominated mode and various mid-width to end width ratio compared to other specimens.

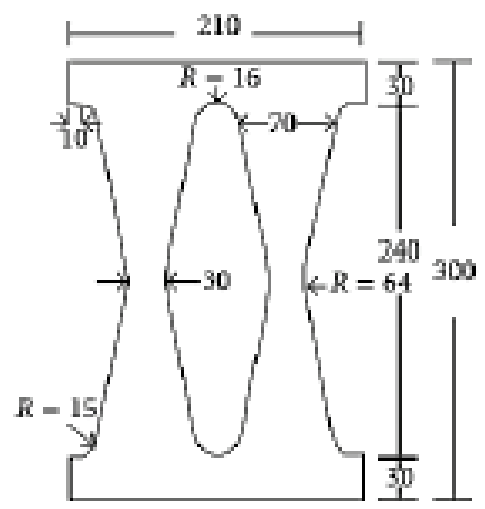

(d) DHSD-4

Figure 2. 26 Dimensions of the recommended specimen [from [14]]

In addition, the location of fracture is investigated, and it is observed that cracks occur in the specimen when the specimen reached its maximum bending moment. The damping ratio is found to be 0.4 for the recommended specimen at a displacement of $20 \mathrm{~mm}$. The damping ratio is investigated through the equations of effective stiffness and effective damping ratio coefficient.

\subsubsection{Parametric Studies of Butterfly-shaped Steel Shear Plates with Various Configurations}

Farzampour and Eatherton [38] have done a research on parametric studies of butterflyshaped shear links with various geometries. To define the shear yielding, flexural yielding and the later torsional buckling limit states of the butterfly-shaped links, a computational study is done through finite element (FE) models with various configurations. Based on these computational 
results the critical moment, the corresponding critical shear force, the location of the plastic hinge and the range of strain hardening, and the energy dissipation amount is studied. One of the goals in the study of energy dissipation devices is considered to be the inelastic deformations and structural damage to the fuses. It is found that the moment capacity is better placed in butterflyshaped links of the moment diagram which would lead to the most efficient use of steel and uniform yielding distribution along the length of the link. It is used between beams and columns to prevent any possible structural damage to beams and columns. Due to the high ductility the fuses are possessing, they can perform under even large shear angles of up to $30 \%$ across the length of link. Under large story displacements butterfly-shaped fuses have proven a high hysteretic response. Based on the laboratory test results, higher ductility and significant initial stiffness have been proven. With that being said, the possibility of brittle limit state should be considered.

Additionally, it is found that as the butterfly-shaped fuses are getting straighter, the location of the plastic hinges approach to the ends of the shear link. However, for the butterfly shaped fuses with a thinner mid-width the geometrical hardening response starts in lower displacements right after the first limit state. The plastic strain values are 5 times higher for butterfly shaped fuses having an $B / C$ (mid-width to end-width) ratio of 0.33 and 0.75 compared to those having a midwidth equal to the $C$ (end-width). This clarifies the possibility of early fracture in straight links. Thickness has a significant effect on the buckling limit state which leads to the amount of energy being dissipated and is independent of the hinge formation location. As the end width of the link increases over the length the shear mechanism will be governing thus resulting in the reduction of the energy dissipation. Considering the plastic strain results, the $B / C$ (mid-width to end-width) ratio equal to $1 / 3$ and $3 / 4$ are recommended because of lower possibility of fracture. For the $1 / 3$ ratio inelasticity is far from the discontinuities as well as resulting lower over-strength, making it desirable for boundary element design purposes.

\subsubsection{Investigating Limit States for Butterfly-shaped Steel Shear Plates}

In another study which has been made by Farzampour and Eatherton [39] the limit states for butterfly shaped fuses have been investigated. In the study, finite element models are created to investigate the better prediction for the controlled shear and flexural limit states. Depending on the butterfly angle either shear or flexure will control. If the angle at the middle of link is greater than 148 degrees, flexure controls and for the inverse inequality shear controls. It is found that if 
shear yielding happens first, there will be less inelastic deformation resulting in reduction of the energy dissipation and less ductility. While for flexural yielding the yielding will be spread out over a large area of the links, thus it will have more capacity for ductility, plastic deformation and energy dissipation. Furthermore, it is concluded that $B / C$ (mid-width to end-width) ratio should be $1 / 3$ to locate the flexural hinges as far as possible from points of stress concentration. For the slit links the shear and flexural controlling depend on the ratio of $C$ (end-width) and $A$ (length). If $C / A$ (end-width to length) is greater than 1.15 then shear controls and for the reverse inequality flexure controls.

\subsubsection{Yielding and Lateral Torsional Buckling Limit States of Butterfly-shaped Steel Shear Plates}

Lateral torsional buckling limit states of butterfly-shaped links have been investigated by Farzampour and Eatherton [40]. The governing differential equation for elastic lateral torsional buckling of a butterfly shaped fuse is derived. The differential equation is formulated based on the equilibrium of torsional moments acting on the deformed configuration of the cross section using twist along the longitudinal axis. When calculating the shear strength of the links lateral torsional buckling, flexural yielding, and shear yielding should be considered. The ratio of lateral torsional buckling strength to the controlling yielding limit state strength can be thought as a measure of how much inelastic deformations are possible prior to buckling. The resulted buckling strength is calculated using the shooting method. Additionally, these results have considered varying geometric parameters. Based on the obtained results, butterfly shaped structural fuses have shown the capacity of dissipating energy substantially.

\subsubsection{Performance of Butterfly-shaped Shear Panels}

In another study which have been made by Koken and Koroglu [24] the use of butterflyshaped shear panels has been investigated. The butterfly-shaped panels have been used in the connections of beam and column. The aim of this study is considered to concentrate the damage on the butterfly shaped panel when the beam to column connection is imposed to the earthquake loading. These fuses in the connections have been considered to undergo long deformations and prevent the damage to the surrounding structural members and after any major event they would be replaced and repaired with minimum cost. The specimen has also computationally analyzed, using solid elements under monotonic loading in the finite element analysis. The computational 
results have matched the experimental ones with an accuracy of $95 \%$. It is found that the energy dissipation of the specimen with butterfly shaped fuses were lower compared to casual end moment connection. The reason for having a lower energy dissipation amount in the butterfly shaped specimen is the less amount of rotation in the connection compared to casual end moment connection. Having said that, the butterfly-shaped specimen has had increased the stiffness and strength of the system compared to simple end moment connection.

\subsection{Comparison of Butterfly-shaped Steel Shear Plates to Slits}

Luth et al. [41] have compared the use of butterfly-shaped fuses to straight ones. They stated that butterfly-shaped fuses show better flexibility due to special geometry properties. As it is stated in their study, if the butterfly-shaped links are designed in such way which the yielding reach its limit states at the end and the middle of the link, the yielding will initiate from the middle and uniformly continue to the ends. In this way $100 \%$ of yielding will occur over the entire length of the link and results in better amount of energy dissipation.

\subsection{Effect of Yield Strength on Steel Shear Plates}

Among several studies which have been made on the energy dissipation ability of steel plates, Katayama et al. [42] stated that steel plates with low yielding point will increase the performance of a structure. It has been mentioned that steel plates with low yielding point will yield earlier than the other surrounding structural components in the system. It is also claimed that these steel plates elongate considerably higher when compared to other structural elements and will increase the ductility of the structure significantly. Due to the strain hardening which consists of kinematic and isotropic hardening, the response of the system will be different under static and dynamic loadings [42].

\subsection{Design and Behavior of Steel Shear Plates}

Ma et al. [13] have studied straight dumpers and butterfly shaped fuses experimentally for further investigations on energy dissipating devices. It is mentioned that the wall with links exhibited ductile behaviour around $5 \%$ shear deformation. However, these fuses can not withstand shear deformations of more than 20 to $30 \%$ due to low cycle fatigue. On the other hand, it is stated that butterfly-shaped fuses can resist a shear deformation of up to $25 \%$ drift ratio. The concept behind using of butterfly-shaped fuses with its special geometry is to align bending capacity with the shape of moment diagram. 
In the study by Ma et al. [13] two new observations, fracture and buckling response of energy dissipating fuses are considered. It is mentioned that fracture might happen in locations where the change of curvature and plastic hinges occur. It is recommended by the author that quarter points should be considered as the areas where the yielding happens. The reason behind choosing this point is stated to be based on the simplification of the behavior of the link.

\subsection{Potential Applications of Structural Steel Shear Plates}

In this section, several potential application of steel shear plates have been discussed. The literature is investigated for each of the application. Some of the many possible systems that a steel shear plate can be implemented is shown in Figure 2.27. Additionally, steel shear plates can also be implemented as structural fuses in seismic resisting systems like self-centering rocking steel braced frame structures and proposed as structural fuses in moment connections.

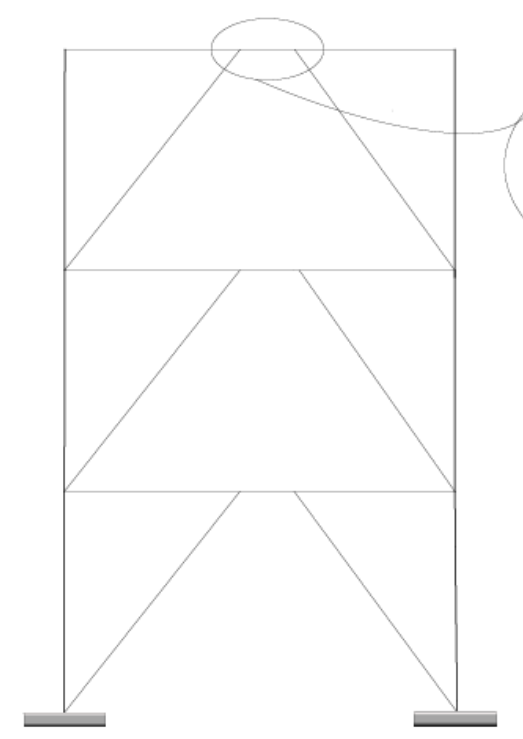

a) Eccentrically Braced Frame Application

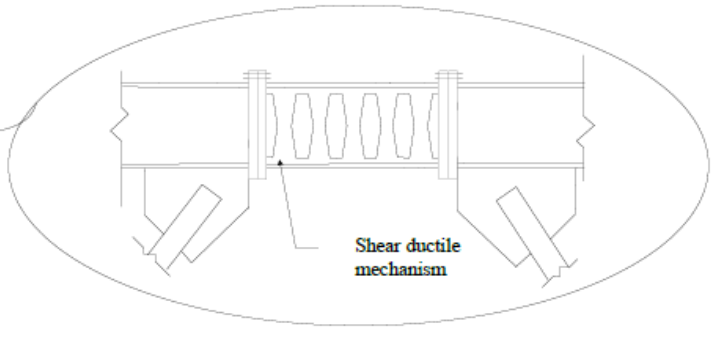

b) Butterfly-shaped fuse

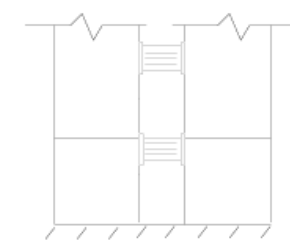

c) Coupled shear wall Application

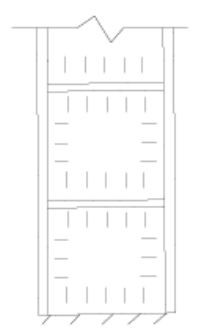

d) Shear wall Application

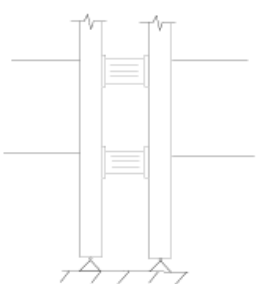

e) Linked column Application

Figure 2. 27 Implementation steel shear plates in different structures [from [16]]

\subsubsection{Steel Shear Plates in Rocking Frames}

Structural fuses are designed to significantly reduce the large structural drifts and to facilitate repairs for the damaged structural elements. These fuses are designed to provide safety, and cost-effective earthquake resistance to the resilient buildings. The steel shear plates with a well detailed design can dissipate energy through shear deformation. Application of buckling resistant 
systems can lead to better use of steel material with no pinching in the dissipation of energy. Implementation of these fuses will result the system to minimize building downtime and the associated disruption following severe earthquakes. These systems are studied thoroughly by a various number of researchers (e.g., [43,44]).

It is worth to mention that the equations or procedures used for controlling the behavior of structural fuses in terms of lateral torsional buckling, flexural and shear yielding limit states are significantly necessary. Since the use of these system is highly sensitive to the buckling limit state, a true prediction of the governing limit state should be accomplished.

\subsubsection{Eccentrically Braced Steel Frames}

Another common type structure used as buckling resistant concept is eccentrically braced steel frames. The braces are eccentric to beam-column connections and they do not directly frame into the connection. The metallic shear fuse indicated in Figure 2.29 is designed to yield during load reversals. For the purpose of desired shear yielding, the steel shear plates' links are designed to have an upper limit to yield in shear for maximizing the energy dissipation.
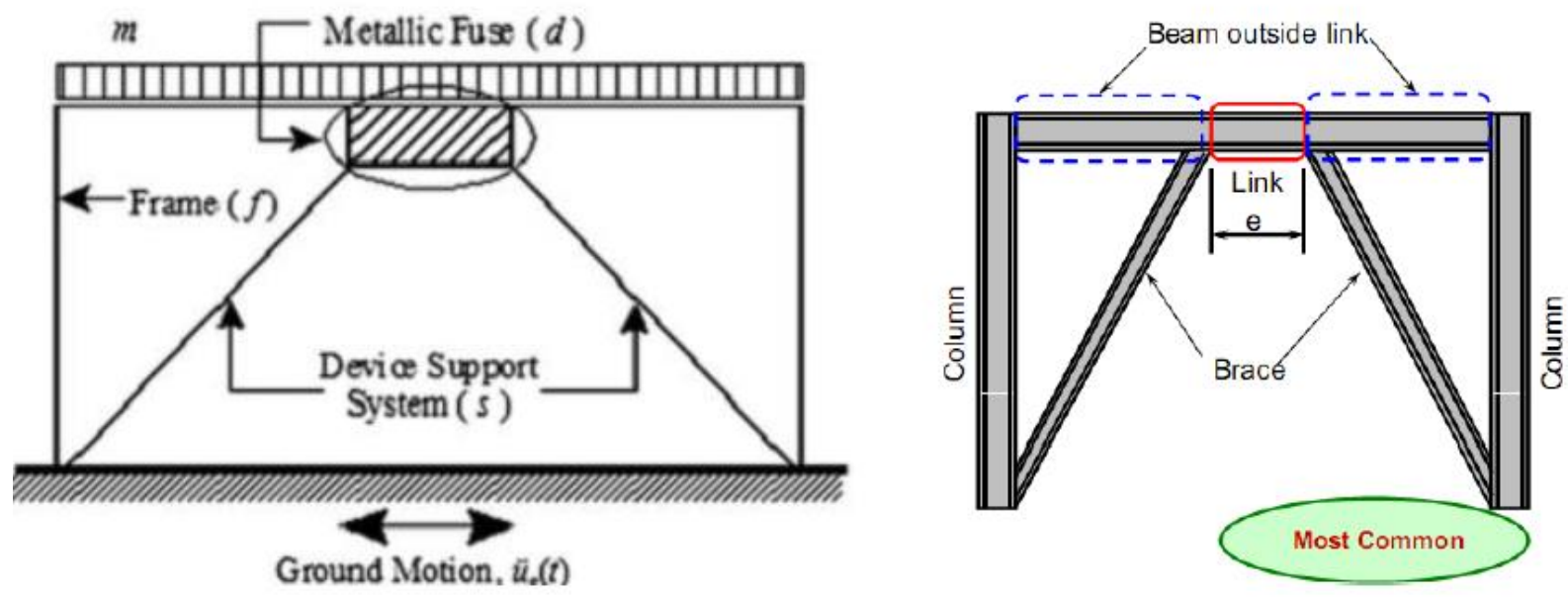

Figure 2. 28 Eccentrically braced frame [from [45]]

According to the fig., if the length of the link is less than $1.6 \mathrm{Mp} / \mathrm{Vp}$ and the rotation of the link is limited to $0.08 \mathrm{rad}$, the spacing of the stiffeners in the system should be less than $30 \mathrm{tw}-$ $d / 5$. In order to achieve an appropriate performance in the eccentrically braced steel frames these requirements under seismic loading conditions should be met. 


\subsubsection{Use of Steel Shear Plates in Bridges}

Another study is made by El-Bahey and Bruneau [46,47] to show the performance of the butterfly-shaped links in the bridge systems. It is concluded that the implementation of butterflyshaped fuses is increasing the stiffness and the strength as well as the amount of energy dissipation of the bridge system as high as $40 \%$ when compared to a bare system. It is mentioned that through various shear links a very stable ductile cyclic behavior can be observed in the system. It is stated that shear links with shorter lengths happen to have uniformly distributed inelastic shear strains, thus allowing the large inelastic deformations without having significant local strains. Also, from another study by El-bahey and Bruneau [46] for the links with longer lengths it is shown that significant bending stresses at the ends will cause large inelastic deformations.

El-Bahey and Bruneau [46] mentioned that buckling is a very significant issue in the design of butterfly-shaped fuses. They stated that if the buckling is prevented in the design of butterflyshaped links, an appropriate and stable hysteric behaviour without significant amount of pinching would be achieved in the system. The authors made an experimental study with three specimens of three different columns, two bare columns, and one steel-plate shear links column, which the butterfly-shaped fuses are used in it. The goal of this experimental study was to further strengthen and observe the concept of structural fuses. As it is earlier described in this study, structural fuses are the sacrificial members used for dissipating energy and keeping the integrity of other main structural components in the system considering the replaceable capability after any major damage in the fuses. It is observed that the BRB and SPSL columns increase the overall strength, stiffness and the amount of energy being dissipated in the system compared to the one with no links in it, while preserving the other structural components in their elastic mode. Their study results show that the column strength is increased by $80 \%$ in SPSL and BRB columns. Furthermore, the stiffness of the column is increased by $31 \%$ and $20 \%$ for SPSL and BRB respectively.

The procedure of best design method and appropriate understanding of which limit state would be governing in the system is not addressed, except for saying that the SPSL is designed based on the curvature of the links. Also, the amount of steel used in the BRB and SPSL columns is not mentioned which would be have a direct relation on the strength and stiffness of the columns compared the one with no links. The cracks are observed in the middle of the links without the consideration of all the limit states. The software and the analytical modeling results were not 
matching with the actual experimental ones due to several factors. Unanticipated strong wall effect, incompatibility of the actual material strength with the designed one, vague experimental changes when the test was running were considered among the reasons which lead to a different result evaluated through analytical and software modeling.

The hysteric curves (Figure 2.30) exhibited a significant amount of stiffness and strength degradation. The experiment was stopped several times for retrofitting the welding which would happen to have uncertain results. Also a few experimental tests were performed on out-of-plane restrained and unrestrained fuses. The restraint condition is achieved through the implementation of fiberglass building material. The two out-of-plane restrained, and unrestrained specimens were observed to have a similar response in terms of yielding and the formation of initial cracks. While the only difference is noticed to be the high amount of pinching in the hysteric curves of unrestrained specimen compare to the restrained one.
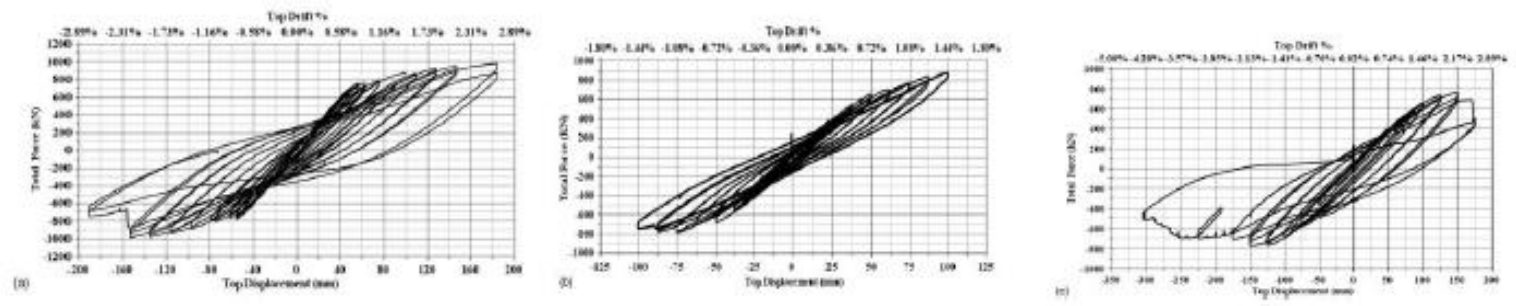

Figure 2. 29 Hysteresis response of specimen [from [46]]

\subsection{Summary}

Steel plate fuses are considered as the main source of energy dissipation in the seismic resistant structures. These fuses are also providing enough ductility to the structures that could lead to the near zero residual drift as well as no main structural damage after any severe ground motion. The significant role of these fuses in different structures opens the doors for a comprehensive research on the effects and their interaction of different design factors on the response characteristics of these fuses. Furthermore, a need to have the optimized shapes of these fuses under different circumstances along with the predictive models for those response characteristics is greatly observed. 


\section{CHAPTER 3 \\ FINITE ELEMENT (FE) MODELING}

\subsection{Overview}

Previously the FE modeling of slit and butterfly-shaped steel plate fuses have been presented in several studies (e.g., [16,37]). In this context, the procedure for numerical analyzing of slit and butterfly-shaped fuses under cyclic loading is thoroughly elaborated. The assumptions and modeling approaches are explored in a detailed manner. Furthermore, the modeling methodology of the computational study is verified under both cyclic and monotonic loading conditions for experimentally tested specimens by Lee et al. [15], and Farzampour [16]. The response of the experimental tests is captured by the computational study, which is related to the out-of-plane deformation as well as the Von Mises stress distribution for different limit states.

\subsection{Reference Slit and Butterfly-shaped Steel Plate Fuses}

In the present study, two specimens from the non-uniform steel strip dampers tested by Lee et al. [15] are chosen for the calibration and verification of FE modeling results under cyclic loading. Further, three different single-link specimens from the numerical study by Farzampour [16] are modeled to confirm the accuracy of the FE analysis results, which is based on the response of a single link of the fuse. These specimens exhibit three different limit states which include flexural yielding, shear yielding, and lateral torsional buckling.

The experimentally tested specimens by Lee et al. [15] include a conventional prismatic strip damper (PSD, which has slit or straight openings) and a dumbbell-shaped damper (DSD also known as butterfly-shaped fuses), as shown in Figure 3.1 and 3.2, respectively. Table 3.1 summarizes the dimensional details of each specimen used for verification purposes. In Table 1, the letter $r$ denotes the radius of the round cut edges at the end of the link and $n$ describes the number of links in the specimen. 


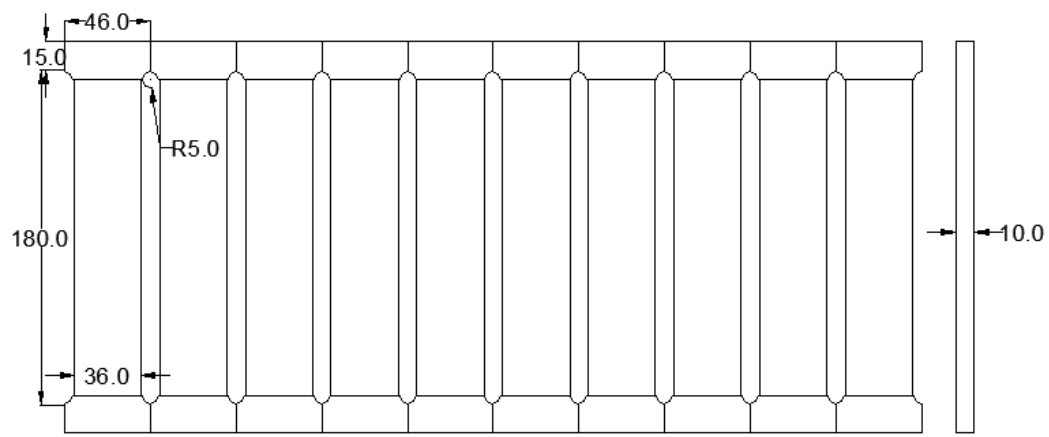

Figure 3. 1 Details of PSD-5 specimen used for the validation study (all dimensions are in $\mathrm{mm}$ )

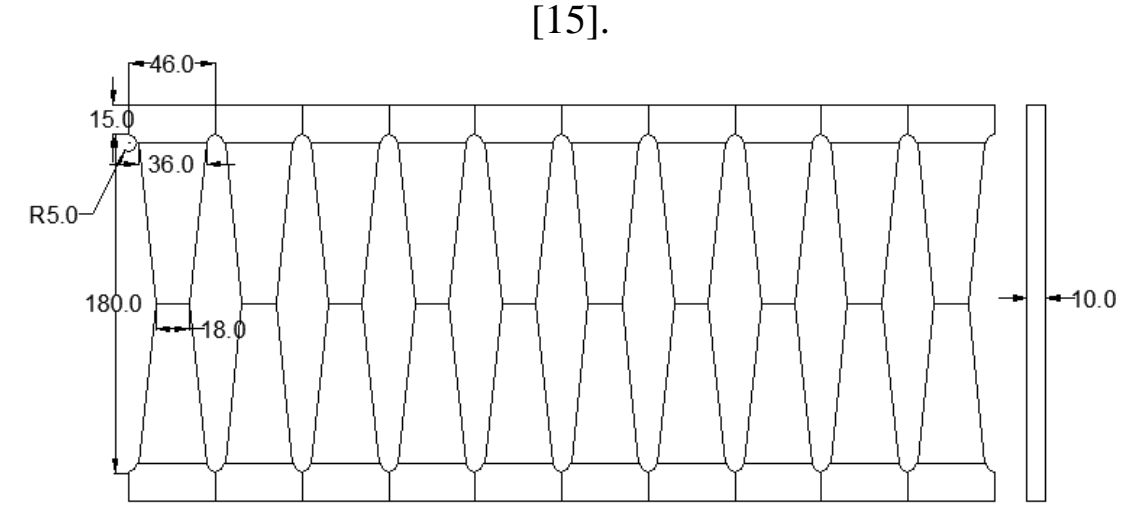

Figure 3. 2 Details of DSD-5 specimen used for the validation study (all dimensions are in $\mathrm{mm}$ ) [15].

Table 3. 1 Dimensional details of the specimens used for the validation study $[15,16]$.

\begin{tabular}{ccccccc}
\hline Specimen/limit state & $A(\mathrm{~mm})$ & $B(\mathrm{~mm})$ & $C(\mathrm{~mm})$ & $D(\mathrm{~mm})$ & $r(\mathrm{~mm})$ & $n$ \\
\hline PSD-5 & 180 & 36 & 36 & 10 & 5 & 10 \\
DSD-5 & 180 & 18 & 36 & 10 & 5 & 10 \\
Shear yielding & 1000 & 100 & 500 & 20 & 5 & 1 \\
Flexural yielding & 1000 & 100 & 300 & 20 & 5 & 1 \\
Lateral torsional & 500 & 100 & 100 & 16.67 & 5 & 1 \\
Buckling & & & & & & \\
\hline
\end{tabular}

\subsection{Development of FE Models}

To evaluate the cyclic response of highly nonlinear steel plate fuses, 3D FE models are developed and analyzed in the FE software, ANSYS Mechanical APDL [48]. Afterward, to 
achieve a satisfactory level of accuracy in the FE simulations, several trial models are investigated. To reduce the computational cost (i.e. running time duration and demand for resources), only one link of the fuse plate is modeled excluding the experimental setup components. Appropriate boundary conditions are directly applied to the fuse model to simulate the proper behavior of the fuse. Figure 3.3 shows the developed model for PSD-5 specimen.

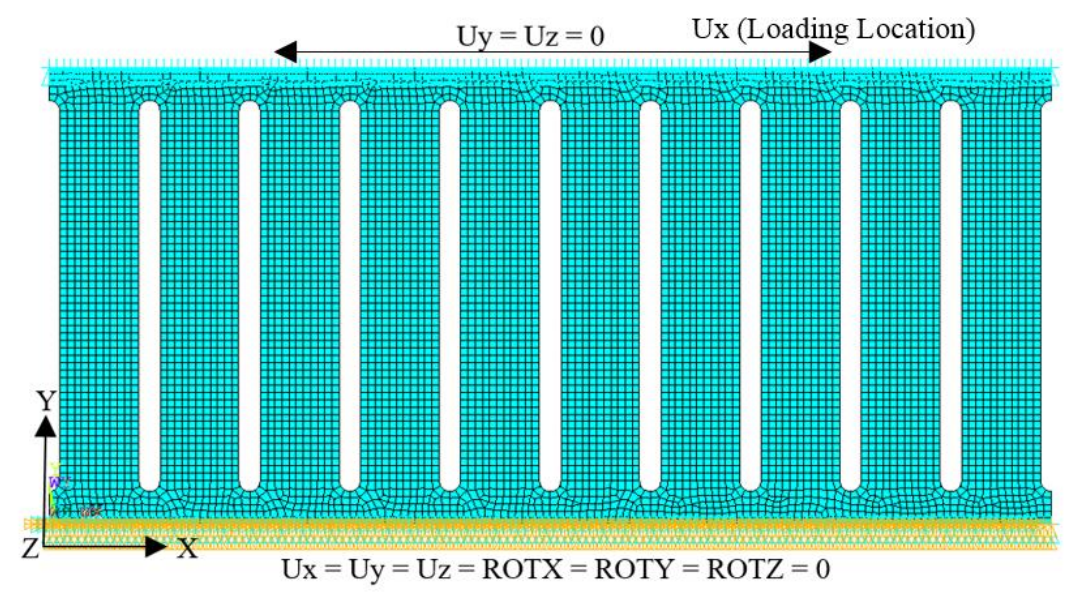

Figure 3. 3 FE model for PSD-5 specimen.

\subsection{Element Type}

Several initial runs using different element types have been explored. Considering the element types, first the analysis results of solid and shell elements such as SHELL281 and SHELL181 are compared with the experimentally tested specimens. These elements are widely used in the FE modelling study of steel plates [49]. It is worth to mention that solid elements are not as accurately capturing the out-of-plane deformations as shell elements. It is observed that shell elements can better capture the behavior of the experimentally tested specimens in terms of loaddeformation response, stress distribution and the out-of-plane deformation.

Among shell elements, SHELL281 and SHELL181 element types are thoroughly studied. Shell elements are suitable for analyzing thin to moderately thick shell structures. These elements are well suited for linear larger rotation, and/or large strain nonlinear applications. Change in the thickness is accounted for in linear and nonlinear analyses. Both element types have shown promising load-deformation responses when compared to the experimentally tested specimens. However, SHELL281, having eight nodes with six degrees of freedom at each node: 3 translational (Ux, Uy, Uz) and 3 rotational (ROTX, ROTY, ROTZ), has shown more computation cost in terms 
of running time when compared to 4-node SHELL181 element type. Therefore, SHELL181 elements with reduced integration are used for the models in this study.

\subsection{Material Properties}

Selection of the right material model is the most challenging part of the validation in the FE simulations. From the ANSYS material library, several types of material models are compared to check the behavior of the model. The load-deformation responses are then compared with the experimental results to find the best matching material model for the numerical simulations. Among the material models, different models including bilinear elastic perfectly plastic, multilinear kinematic hardening model, the NLISO model (Chaboche model combined with nonlinear isotropic hardening) and combined isotropic and kinematic hardening model have been used and compared. As a result of these comparisons, NLISO model (Chaboche model combined with non-linear isotropic hardening) is used as the material model in this study. Values of $200 \mathrm{GPa}$ and $295 \mathrm{MPa}$ are used as the elastic modulus of elasticity and yield stress of steel, respectively, based on the coupon tests reported in Lee et al. [15]. For some specimens of different experimental tests higher values of yield strength (359 MPa )is considered for FE modeling.

The NLISO material model is especially suitable for large-deformation analyses. Three different constant values, including $R_{0}, R_{\infty}$, and $b_{0}$ should be specified when using this material model. These values characterize the isotropic hardening behavior of the material. $R_{0}$ is the initial isotropic hardening modulus while $R_{\infty}$ is the asymptotic hardening value. $b_{0}$ controls the decrease of hardening in the function of equivalent plastic strain. For the verified models, values of 16, 30 and 50 are used for $R_{0}, R_{\infty}$ and $b_{0}$, respectively [50].

\subsection{Boundary Conditions}

In the experimental test setup by Lee et al. [15], the cyclic performance of steel strip dampers have been evaluated using a quasi-static $500 \mathrm{kN}$ actuator. The loading has been applied by displacement control through the inner displacement transducer. Pin-connected fixing rods have been installed at two sides of the specimen. Therefore, the fixing rods are restraining the vertical displacement of the testing frame and the loading can be induced at the top edge of the horizontal beam which is connected to the specimen. In the FE model, the bottom of the plate at the bolt line is fixed against out-of-plane as well as vertical translation whereas a roller reaction at the top edge of the specimen is assumed where it can only move in the direction of loading and is constrained 
against out-of-plane and vertical translation. The load in the FE model is applied at the top edge of the plate. It is also worth mentioning that the band zone areas at the top and the bottom of the plate are modeled to better capture the stiffness of the model. The reader is kindly referred to Figure 3.3 for the FE model with the proper boundary conditions.

\subsection{Meshing Properties}

The mesh density of the FE is model is controlled using mesh control features in ANSYS Mechanical APDL [48]. Using mesh control features, line and element sizes are assigned and an automated free mesh is generated for the entire model. Free meshing provides the most desirable regular meshing in irregular shapes. Figure 3.4 shows the meshing for the FE model of specimen DSD-5. Creating a fine enough and regular mesh is vital for capturing the proper cyclic response and overcoming possible convergence problems.

A mesh sensitivity study is carried out and based on the results, an element size of $3.5 \mathrm{~mm}$ is used for the fuse model. This mesh size results in having 6, 20 and 46 element over the midwidth, end-width and length, respectively, following the meshing recommendations by previous researchers [16]. Having this meshing property, for instance, DSD-5 specimen consists of 108 keypoints, 152 lines, 40 areas, 6662 nodes, and 5944 elements. It is worth to mention that a specimen of these properties takes around 5 hours and 25 minutes to have a complete run on a computer with 16 gigabytes RAM and process of $3.70 \mathrm{GHz}$ (12 CPUs). It is confirmed that imperfection is not significantly influencing the response of steel fuse models. Thus, no imperfection is introduced in the models.

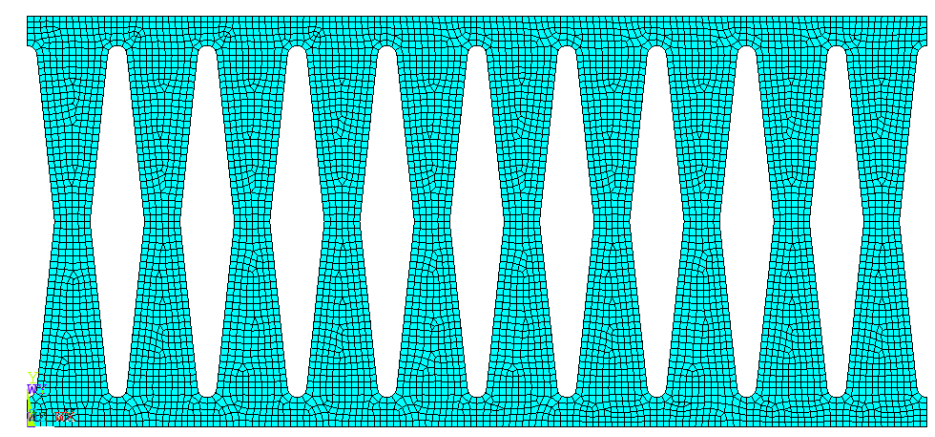

Figure 3. 4 Meshing details of specimen DSD-5. 


\subsection{Loading Profile}

To find the accumulated damage in the models due to the hysteretic loading, displacementcontrolled cyclic loading with incremental amplitudes similar to the experimental setup by Lee et al. [15], is applied. The loading protocol consists of symmetrical cyclic drift ratio with incrementally increasing amplitudes. The incremental variation for the amplitudes of first 12 steps of loading is increased with a ratio of 1.40 . For instance, in the loading profile, the $1^{\text {st }}$ load step is set to $0.44 \mathrm{~mm}$ and the $2^{\text {nd }}$ load is obtained by the multiplication of 1.40 and 0.44 (i.e., the second load step $=0.62 \mathrm{~mm}=1.40 \times 0.44 \mathrm{~mm}$ ). Following the $12^{\text {th }}$ load step, the increase in the increments have been fixed at $5.40 \mathrm{~mm}$. Figure 3.5 shows the loading history.

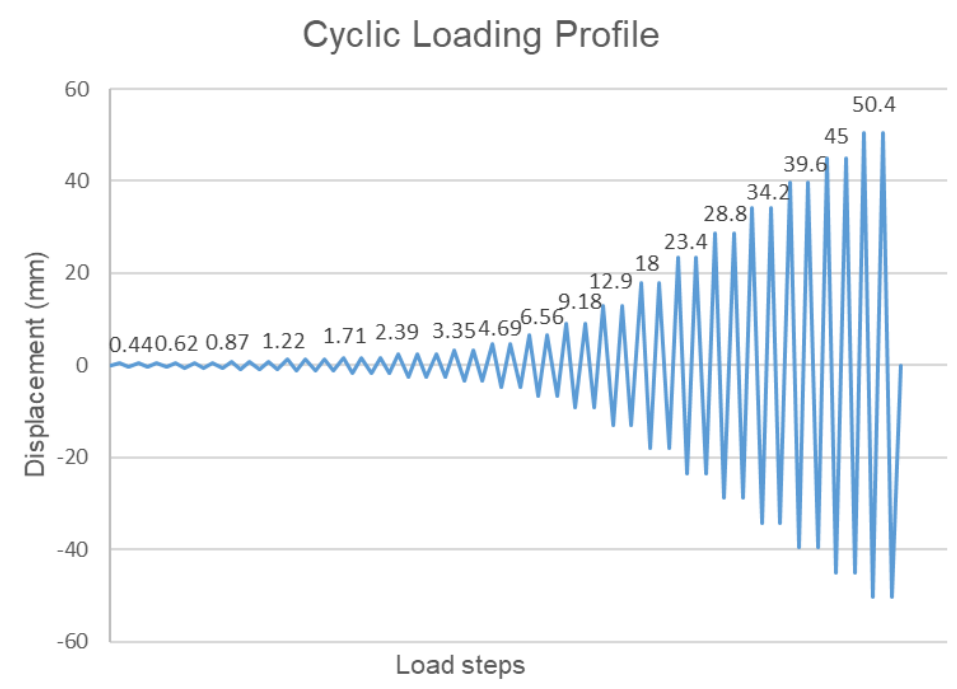

Figure 3. 5 Cyclic loading history

\subsection{Analysis Method}

Nonlinear static analyses with full Newton-Raphson solution scheme considering the geometric and material nonlinearities are performed in ANSYS Mechanical APDL [48]. The Newton-Raphson is used to rapidly find an appropriate approximation for the root of a real-valued function $f(x)=0$. This method utilized the concept that a continuous and differentiable function can be approximated by a straight line which is tangent to it. Geometric and material nonlinearities are considered to capture the real-behavior of the steel plate in its plastic region. In order to 
overcome convergence difficulties, the minimum number of sub-steps to be taken is set to 10 and 25 for small and large amplitude load steps, respectively.

\subsection{Validation of FE Modeling}

This section presents the validation of the FE modeling for different specimens, including two steel plate fuses among those specimens tested by Lee et al. [15]. The selected specimens include DSD-5 and PSD-5 specimens, which are a dumbbell-shaped and a prismatic fuse, respectively. Note that dumbbell-shaped fuses are also known as butterfly-shaped fuses, and prismatic fuses are those with slit or straight openings [13,39,40,51]. Figure 3.6 shows the lateral load versus the lateral displacement of specimens DSD-5 and PSD-5 in comparison with the experimental response curves. As shown in Figure 3.6, the numerical results are in good agreement with the cyclic test results. The error in predicting the peak strength using the FE model is $1.14 \%$ and $3.50 \%$ for specimens PSD-5 and DSD-5, respectively. The error percentage here is obtained from $\frac{P_{\max }^{F E}-P_{\max }^{T e s t}}{P_{\max }^{T e s t}} \times 100$, where $P_{\max }^{F E}$ and $P_{\max }^{T e s t}$ are the peak strength from the FE analysis and the experimental test, respectively. Considering the displacement, since the full experimental assembly is not modeled, a comparatively low error percentage for the displacement is expected.
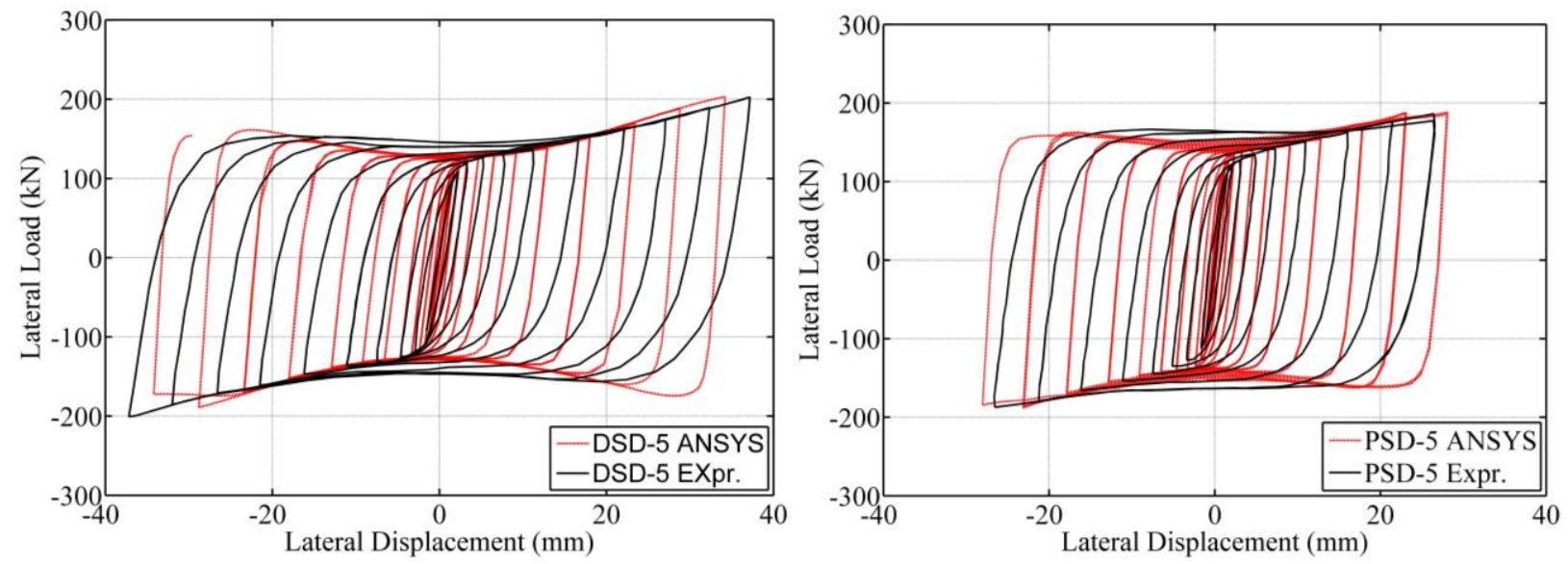

Figure 3. 6 Cyclic load-displacement response of specimens DSD-5 and PSD-5: FE simulation results in comparison with experimental results.

The accuracy of the FE analysis in predicting the limit states of butterfly-shaped fuses is also verified against three specimen models analyzed by Farzampour [16]. The detailed dimensions of the models experiencing flexural yielding, shear yielding, and lateral torsional 
buckling is listed in Table 3.1. These specimens include only one link of the dumbbell-shaped (i.e., butterfly-shaped) and slit (i.e., prismatic) fuses due to the computational costs. For this reason, as this research is also considering only one link of the fuse, the FE model in this study is validated with the reference numerical results. Note that the numerical results (ABAQUS) in the reference study are validated with experimentally tested specimen [16]. It is worth to mention that there is no evidence on experimentally tested one link specimen of either butterfly-shaped (dumbbellshaped) or slit (prismatic) fuses in the literature. The lateral load-displacement plots of these three specimens are compared and shown in Figure 3.7. The reason for the difference at the end of deformation of each validation plot is believed to be the meshing properties. The reference study has used a coarser mesh (using explicit solver) when compared to the current studies meshing properties (using static solver).

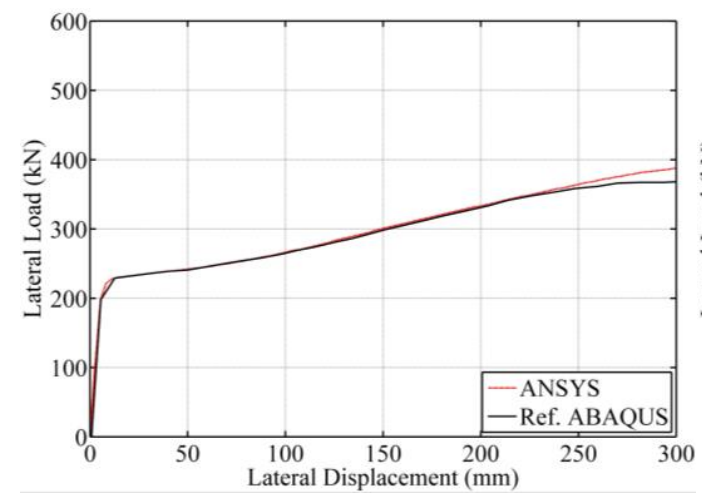

(a)

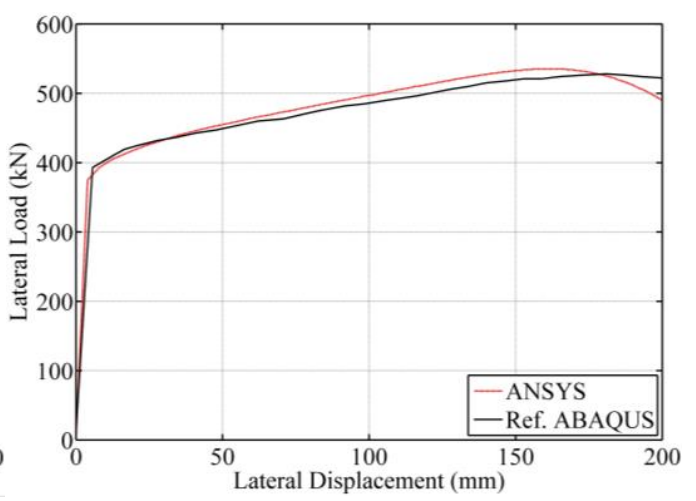

(b)

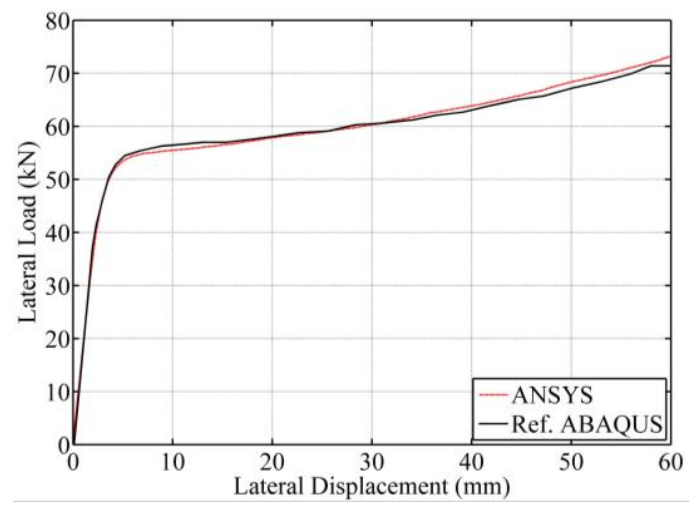

(c)

Figure 3. 7 Validation of the FE modeling against a numerical reference study of limit states [16]: (a): flexural yielding, (b): shear yielding, (c): lateral torsional buckling. 


\subsection{Failure Criteria}

The onset of fracture prediction in FE modelling is a challenging task due to the low-cycle fatigue behavior of structural elements. Various studies have investigated different methods to come up with an appropriate and consistent method to predict the onset of fracture in FE modeling for steel plates. Among those, Ohasaki and Nakajima [52] used the ratio of equivalent plastic strain to the critical plastic strain as the failure index. Others suggested the ratio of Von Mises strain to the ultimate strain of the material as an appropriate approach for defining the onset of fracture.

The use of maximum equivalent plastic strain is suggested by many researchers as an appropriate failure index for steel plates [52-54]. For this study, the maximum equivalent plastic strain is considered as the failure index. To validate the correct value of the maximum equivalent plastic strain several values from different experimentally tested specimens are considered. Hedayat [49], provided the maximum equivalent plastic strain values of different experimentally tested steel plate fuses by Chan et al. [20]. These specimens included butterfly-shaped and slit specimens. An average value of 5.1 was considered as the onset fracture for the experimentally tested specimens. The yield strength of material of the specimens was $317 \mathrm{MPa}$, a comparatively similar yield strength of the material employed in this study. It was observed that, this value happened to be consistent with the fracture location of experimentally tested specimens.

According to this study a maximum equivalent plastic strain of 5.0 is considered as the failure index in this study. To confirm this approach, the location, load-deformation plot as well as the out-of-plane deformation of one of the butterfly-shaped experimentally tested specimens is validated in this study. The deformed shape of the specimen is shown in Figure 3.8 and is compared to the FE model. The comparison of the figures shows that, the behavior of the tested specimen is well captured in the FE modeling. Furthermore, it is observed in Figure 3.9, that the equivalent plastic strain value occurs to be around five at a shear deformation in which the experimentally tested specimen was fractured. The load-deformation plot for this specimen is shown in Figure 3.10 . 

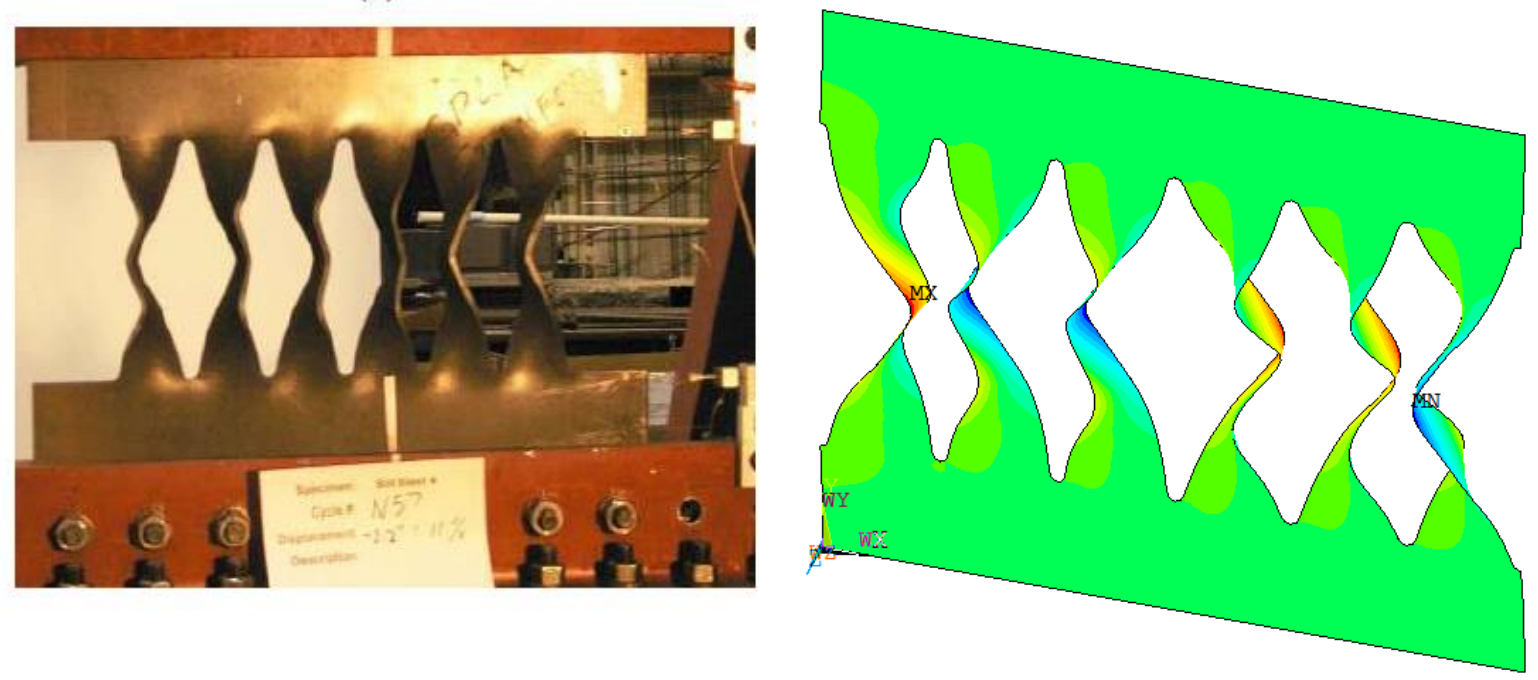

Figure 3. 8 Comparison of the Validated B10-36 Specimen with FE model [from [13]]

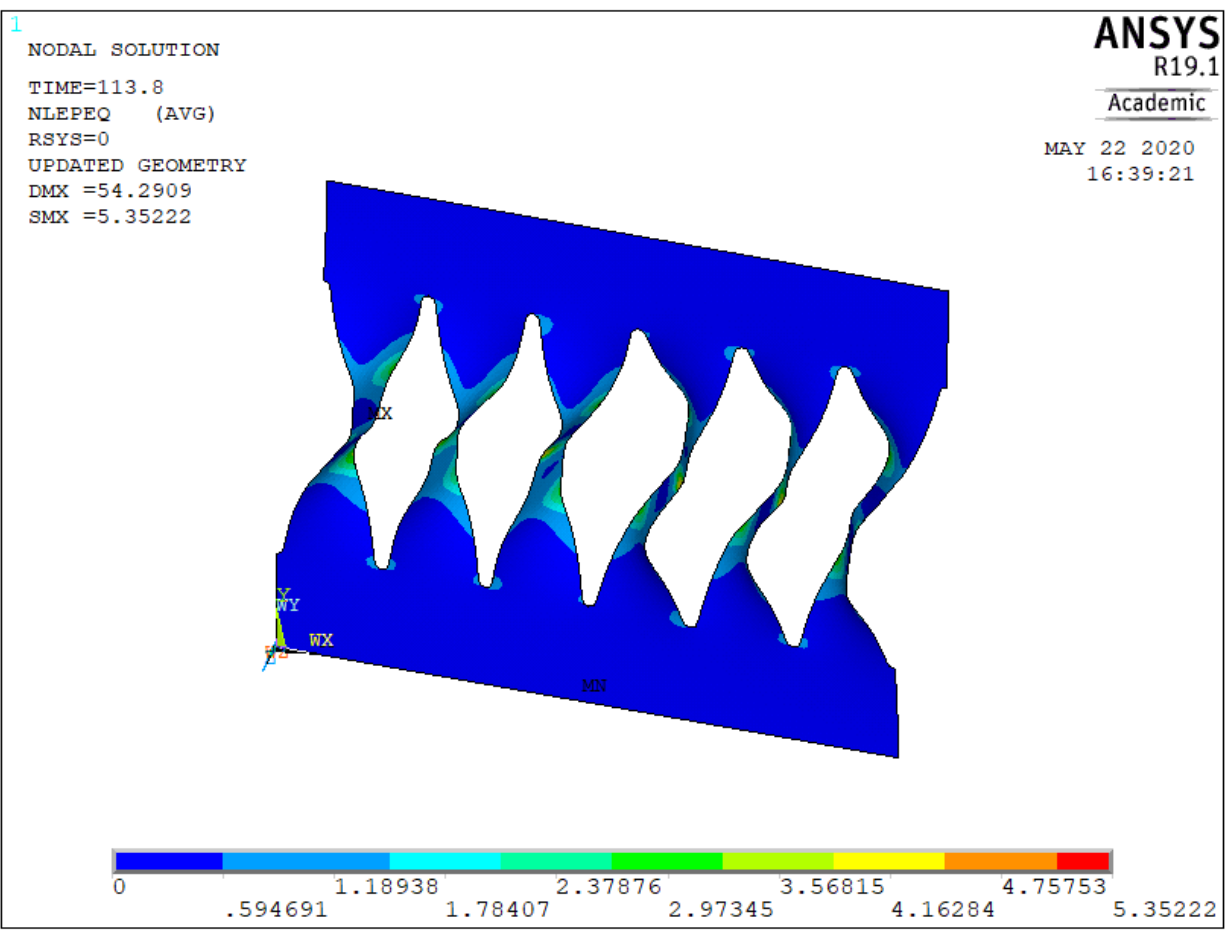

Figure 3. 9 Equivalent plastic strain distribution in the buckled FE model. 


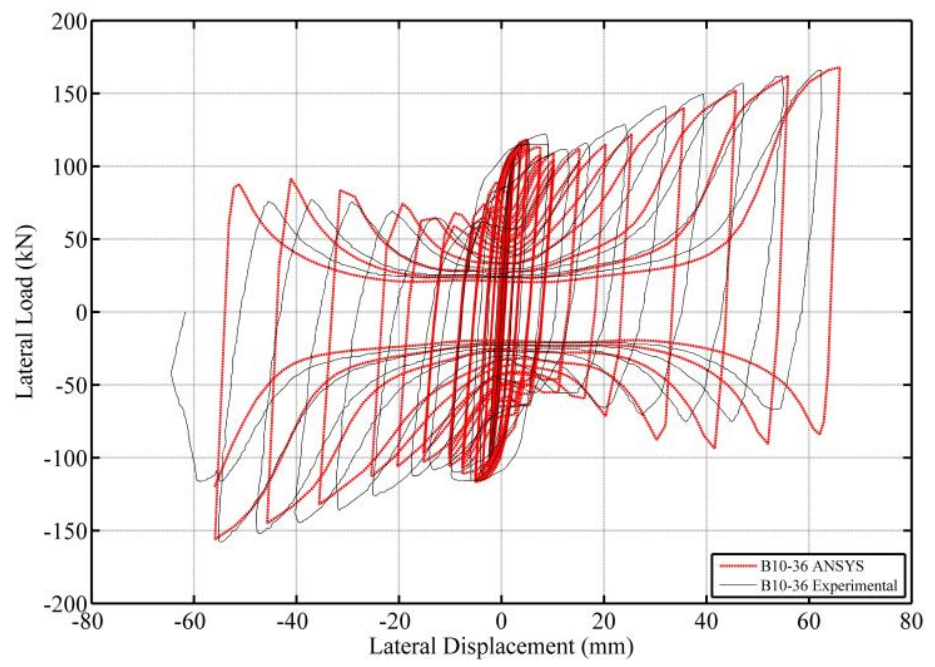

Figure 3. 10 Load-deformation comparison of cyclic response of B10-36 specimen and FE model. 


\section{CHAPTER 4}

\section{SENSITIVITY ANALYSIS OF ENERGY DISSIPATING STEEL PLATE FUSES}

\subsection{Overview}

A sensitivity analysis of the cyclic load-displacement response of steel plate fuses is conducted to assess the effects of different varying design parameters based on the statistical design of experiment; a method that deals with planning, analyzing, and performing controlled tests to evaluate the factors that control the value of a parameter or group of parameters; and the validated FE models. The method of carrying a sensitivity study of a number of design factors on different response variables using design of experiment is used by different researchers (e.g., [55]). Seven potentially influential factors on the cyclic response characteristics are considered. Five of the factors are related to the fuse geometry, including the fuse length, $A$; mid-width, $B$; endwidth, $C$; thickness, $D$; and band zone length, $c$. The remaining two factors are material-related factors, including yield strength of the steel, $F_{y}$; and modulus of elasticity, $E$. Two levels are considered for the input factors. The reason behind selecting only two levels is that the sole purpose of the study is to investigate the main effects of several factors, simultaneously. Therefore, these designs with orthogonal two levels are reliable enough to achieve the goal of screening phase with greatly simplified analysis. Table 4.1 lists these factors along with their low (-) and high levels (+). The factor ranges are selected to be broad and practical based on previous experimental studies (e.g., $[13,15])$.

\subsection{Design of Experiment}

The sensitivity analysis is carried out using the Design-Expert software [56], a statistical software package which offers comparative tests, screening, characterization, optimization using analysis of variance [56]. A two-level fractional factorial design is considered for the analysis. A full factorial design with seven factors and two levels for each factor requires analyzing $2^{7}(=128)$ models, which is computationally expensive. Therefore, a one-fourth fractional factorial design with a resolution of four has been employed in this study. By doing this, the number of runs is reduced to $2^{7-2}(=32)$. It is noted that 32 or $\left(2^{5}\right)$ runs is for a full factorial design with five factors including all possible combinations of high and low levels. Since seven factors are presented in the design, two generators (=7-2) are needed to assign the low or high level for the remaining factors. These two generators are; $E=(A)(B)(C)(D)$ and $c=(A)(B)(C)\left(F_{y}\right)$ [56]. In this case, 
the high and low levels of factor $E$ is specified by the multiplication of $A, B, C$ and $D$. Table 4.2 lists the factor combinations for each run. For each combination of factors, a FE model has been created and analyzed under cyclic loading.

Table 4. 1 Factors and ranges considered for the sensitivity analysis.

\begin{tabular}{ccccc}
\hline Factors & Symbol & Low level (-) & High level (+) & Units \\
\hline Length & $A$ & 180 & 500 & $\mathrm{~mm}$ \\
Mid-width & $B$ & 15 & 36 & $\mathrm{~mm}$ \\
End-width & $C$ & 36 & 200 & $\mathrm{~mm}$ \\
Thickness & $D$ & 5 & 40 & $\mathrm{~mm}$ \\
Steel yield strength & $F_{y}$ & 245 & 340 & $\mathrm{MPa}$ \\
Modulus of & $E$ & 185000 & 212000 & $\mathrm{MPa}$ \\
elasticity & & 5 & & $\mathrm{~mm}$ \\
Band zone length & $c$ & 5 & & \\
\hline
\end{tabular}

As the output of each simulation, six different response variables have been recorded from the cyclic load-displacement response. These response parameters include: (1) the initial stiffness, $K$; (2) yield strength, $P_{y}$; (3) ultimate stiffness, $K$; (4) effective damping, $\beta_{\text {eff }}$; (5) maximum strength, $P$; and (6) ductility, $\mu$. Table 4.3 lists the response values obtained from the nonlinear analysis of each model.

Effective stiffness $\left(k_{\text {eff }}\right)$ is calculated from Eq. 1, where the positive and negative peak displacements $\left(\delta^{+}\right.$and $\left.\delta^{-}\right)$and the corresponding loads $\left(P^{+}\right.$and $\left.P^{-}\right)$are used. The effective damping is used to convert the hysteretic energy of a damper into an equivalent linear viscous system. Effective damping ( $\beta_{\text {eff }}$ ) is used to define the nonlinear behavior of a displacement dependent device [51]. It describes the energy dissipation capacity of a fuse and is calculated using Eq. 2, in which $E_{\text {loop }}, k_{\text {eff }}, \delta^{+}$and $\delta^{-}$define the hysteresis energy, equivalent stiffness, positive and negative peak displacements under the load-displacement plot, respectively. 


$$
k_{\mathrm{eff}}=\frac{\left(\left|P^{+}\right|+\left|P^{-}\right|\right)}{\left(\left|\delta^{+}\right|+\left|\delta^{-}\right|\right)}
$$

$$
\beta_{\mathrm{eff}}=\left(\frac{2}{\pi}\right) \frac{E_{\text {loop }}}{\left(k_{\mathrm{eff}}\right) *\left(\left|\delta^{+}\right|+\left|\delta^{-}\right|\right)^{2}}
$$

Initial stiffness $(K)$ is calculated as the ratio of yield strength to yield displacement while ultimate stiffness $\left(K^{\prime}\right)$ is calculated from the slope of the last two steps of the response curve. Further, the ductility is expressed as the ratio of maximum displacement to yield displacement. Lastly, maximum load $(P)$ is considered as the maximum strength of the fuse, while the first occurrence of yielding is taken as the yield strength $\left(P_{y}\right)$.

\begin{tabular}{|c|c|c|c|c|c|c|c|}
\hline Model & $\begin{array}{c}A: \\
\text { Length } \\
\text { mm }\end{array}$ & $\begin{array}{c}B: \text { Mid- } \\
\text { width } \\
\text { mm }\end{array}$ & $\begin{array}{c}C: \text { End- } \\
\text { Width } \\
\text { mm }\end{array}$ & $\begin{array}{c}D: \\
\text { Thickness } \\
\text { mm }\end{array}$ & $\begin{array}{c}F_{y}: \text { Steel } \\
\text { yield strength } \\
\mathrm{MPa}\end{array}$ & $\begin{array}{c}E: \text { Modulus } \\
\text { of elasticity } \\
\mathrm{MPa}\end{array}$ & $\begin{array}{c}c: \text { Band } \\
\text { zone length } \\
\mathrm{mm}\end{array}$ \\
\hline 1 & 180 & 36 & 200 & 40 & 245 & 185000 & 75 \\
\hline 2 & 180 & 36 & 200 & 40 & 340 & 185000 & 5.0 \\
\hline 3 & 180 & 36 & 36.0 & 40 & 340 & 212000 & 75 \\
\hline 4 & 180 & 15 & 200 & 5.0 & 245 & 185000 & 5.0 \\
\hline 5 & 500 & 15 & 36.0 & 40 & 245 & 212000 & 5.0 \\
\hline 6 & 500 & 15 & 36.0 & 5.0 & 245 & 185000 & 5.0 \\
\hline 7 & 180 & 36 & 36.0 & 40 & 245 & 212000 & 5.0 \\
\hline 8 & 500 & 36 & 36.0 & 40 & 340 & 185000 & 5.0 \\
\hline 9 & 180 & 15 & 36.0 & 5.0 & 340 & 212000 & 5.0 \\
\hline 10 & 180 & 15 & 36.0 & 5.0 & 245 & 212000 & 75 \\
\hline 11 & 180 & 36 & 36.0 & 5.0 & 245 & 185000 & 5.0 \\
\hline 12 & 180 & 15 & 200 & 40 & 340 & 212000 & 75 \\
\hline 13 & 180 & 36 & 200 & 5.0 & 340 & 212000 & 5.0 \\
\hline 14 & 500 & 36 & 36.0 & 5.0 & 340 & 212000 & 5.0 \\
\hline 15 & 500 & 36 & 36.0 & 5.0 & 245 & 212000 & 75 \\
\hline 16 & 500 & 15 & 36.0 & 40 & 340 & 212000 & 75 \\
\hline
\end{tabular}

Table 4. 2 Factor combination for each model. 


\begin{tabular}{|c|c|c|c|c|c|c|c|}
\hline 17 & 180 & 15 & 200 & 5.0 & 340 & 185000 & 75 \\
\hline 18 & 500 & 36 & 200 & 5.0 & 245 & 185000 & 5.0 \\
\hline 19 & 500 & 36 & 200 & 5.0 & 340 & 185000 & 75 \\
\hline 20 & 500 & 15 & 200 & 5.0 & 340 & 212000 & 5.0 \\
\hline 21 & 180 & 15 & 36.0 & 40 & 245 & 185000 & 75 \\
\hline 22 & 500 & 15 & 200 & 40 & 245 & 185000 & 75 \\
\hline 23 & 180 & 15 & 200 & 40 & 245 & 212000 & 5.0 \\
\hline 24 & 180 & 36 & 36.0 & 5.0 & 340 & 185000 & 75 \\
\hline 25 & 500 & 15 & 200 & 40 & 340 & 185000 & 5.0 \\
\hline 26 & 500 & 36 & 200 & 40 & 245 & 212000 & 5.0 \\
\hline 27 & 500 & 36 & 36.0 & 40 & 245 & 185000 & 75 \\
\hline 28 & 500 & 36 & 200 & 40 & 340 & 212000 & 75 \\
\hline 29 & 500 & 15 & 36.0 & 5.0 & 340 & 185000 & 75 \\
\hline 30 & 180 & 15 & 36.0 & 40 & 340 & 185000 & 5.0 \\
\hline 31 & 180 & 36 & 200 & 5.0 & 245 & 212000 & 75 \\
\hline 32 & 500 & 15 & 200 & 5.0 & 245 & 212000 & 75 \\
\hline
\end{tabular}

Table 4. 3 Response characteristics of each model.

\begin{tabular}{ccccccc}
\hline Model & $\begin{array}{c}\text { Initial } \\
\text { Stiffness }\end{array}$ & $\begin{array}{c}\text { Yield } \\
\text { Strength }\end{array}$ & $\begin{array}{c}\text { Ultimate } \\
\text { Stiffness } \\
\text { kN/mm }\end{array}$ & $\begin{array}{c}\text { Effective } \\
\text { Damping }\end{array}$ & $\begin{array}{c}\text { Maximum } \\
\text { Strength } \\
\mathrm{kN} / \mathrm{mm}\end{array}$ & Ductility \\
\hline 1 & 572.97 & 212.0 & 5.19 & 2600.0 & 565 & 107.03 \\
2 & 959.38 & 307.0 & 2.04 & 2310.0 & 663 & 106.88 \\
3 & 18.070 & 30.90 & 2.02 & 43600 & 202 & 76.842 \\
4 & 79.333 & 11.90 & 0.27 & 346.00 & 26.0 & 86.000 \\
5 & 1.6887 & 8.680 & 0.40 & 10400 & 50.7 & 19.260 \\
6 & 0.1851 & 1.090 & 0.03 & 354.00 & 2.03 & 3.9728 \\
7 & 54.545 & 24.00 & 0.37 & 14300 & 131 & 200.45 \\
8 & 2.6459 & 13.60 & 0.18 & 55000 & 99.0 & 36.070 \\
9 & 3.9444 & 4.260 & 0.09 & 344.00 & 6.97 & 11.944 \\
10 & 1.8278 & 2.760 & 0.03 & 330.00 & 4.86 & 11.818
\end{tabular}




\begin{tabular}{|c|c|c|c|c|c|c|}
\hline 11 & 5.8000 & 3.190 & 0.11 & 646.00 & 6.86 & 32.727 \\
\hline 12 & 510.71 & 143.0 & 5.19 & 1420.0 & 405 & 102.85 \\
\hline 13 & 134.29 & 37.60 & 0.73 & 550.00 & 63.0 & 64.286 \\
\hline 14 & 0.3730 & 1.750 & 0.02 & 962.00 & 3.80 & 7.2920 \\
\hline 15 & 0.2412 & 1.240 & 0.05 & 78.300 & 1.89 & 2.5097 \\
\hline 16 & 1.3398 & 12.30 & 0.06 & 11300 & 59.5 & 11.372 \\
\hline 17 & 55.312 & 17.70 & 1.25 & 34.200 & 25.2 & 10.469 \\
\hline 18 & 15.000 & 18.30 & 0.82 & 173.00 & 28.8 & 10.574 \\
\hline 19 & 11.590 & 27.70 & 0.64 & 111.00 & 38.5 & 5.3975 \\
\hline 20 & 11.229 & 13.70 & 0.30 & 29.300 & 18.1 & 3.8442 \\
\hline 21 & 12.748 & 21.80 & 0.67 & 4280.0 & 58.5 & 29.474 \\
\hline 22 & 66.481 & 71.80 & 1.15 & 3020.0 & 198 & 41.667 \\
\hline 23 & 706.67 & 106.0 & 2.04 & 1190.0 & 327 & 192.00 \\
\hline 24 & 1.9037 & 4.550 & 0.08 & 642.00 & 6.97 & 9.7908 \\
\hline 25 & 79.344 & 96.80 & 0.13 & 3260.0 & 283 & 72.295 \\
\hline 26 & 136.06 & 166.0 & 2.59 & 13100 & 372 & 63.442 \\
\hline 27 & 1.6768 & 11.00 & 0.37 & 17300 & 62.0 & 17.561 \\
\hline 28 & 106.13 & 225.0 & 1.57 & 14800 & 490 & 39.056 \\
\hline 29 & 0.1488 & 1.690 & 0.06 & 139.00 & 2.68 & 2.0617 \\
\hline 30 & 27.705 & 33.80 & 0.39 & 19600 & 106 & 76.721 \\
\hline 31 & 83.125 & 26.60 & 0.38 & 394.00 & 46.4 & 40.133 \\
\hline 32 & 9.6226 & 10.20 & 1.18 & 4.5300 & 12.8 & 2.2547 \\
\hline
\end{tabular}

\subsection{Results of the Sensitivity Analysis}

The load-displacement cyclic response of each model is recorded. A wide range of cyclic response is observed. For example, the cyclic response plots for three models 1, 7, and 12 are illustrated in Figure 4.1(a), (b), and (c). Model 7 with the factor combinations shown in Table 4.2 has shown greater ductility compared to models 1 and 12 . The obtained results for the response characteristics of these three models show that the effective damping in model 7 is respectively 10 times and 5.50 times higher than those in models 1 and 12. Maximum strength of model 1 is 4.30 
and 1.40 times greater than that in models 1 and 12, respectively. Considering the ductility, model 7 shows better results when compared to other two models, 1 and 12. Lastly, yield strength response is considerably higher in model 1 when compared to model 7 and 12 . The sensitivity analysis results will be subsequently presented along with the discussions of the effect of the design factors on the cyclic response characteristics of fuses.

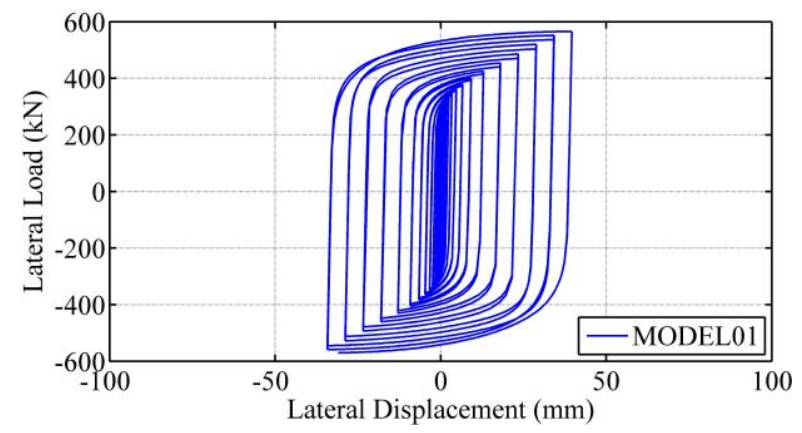

(a)

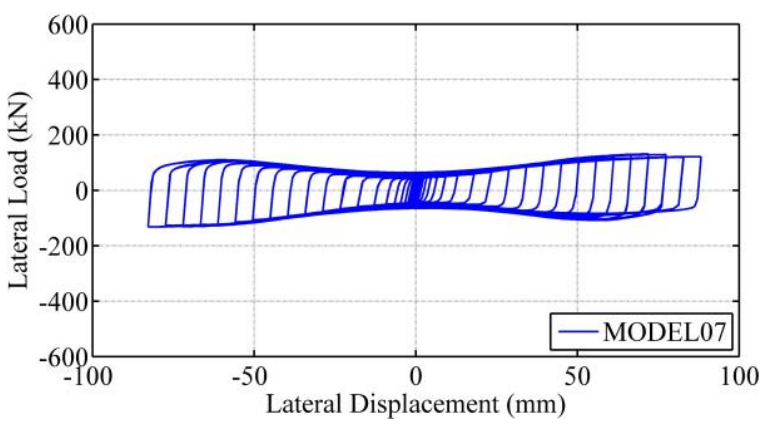

(b)

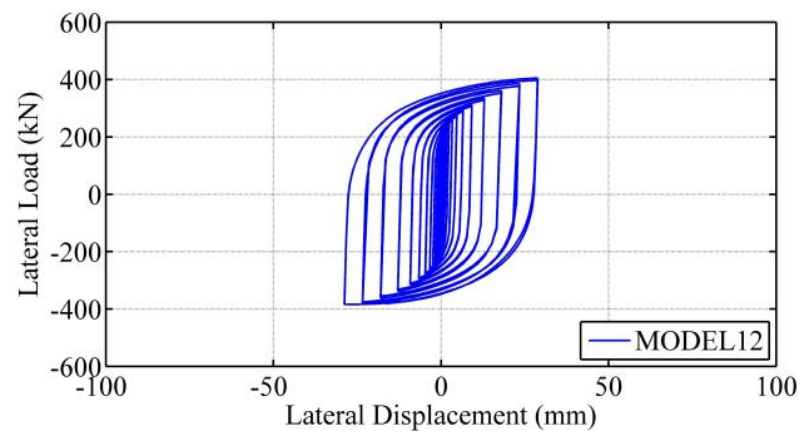

(c)

Figure 4. 1 Hysteretic response plots for: (a) model 1; (b) model 7; (c) model 12.

Important factors and interactions that have significant effects on the cyclic response are identified by a half-normal probability plot. This method is based on the theory that insignificant factors fall on a straight line and are normally distributed with a near-zero mean value [57]. After generating the half-normal probability plot for each response variable, we obtain the effects of main factors and their interactions.

In order to check the significance of the statistical model for each response variable, statistical analysis of variance (ANOVA) using the Design-Expert software is performed [56]. The results from half-normal probability plots are also confirmed. ANOVA is a method to statistically 
test the null hypothesis of no treatment effects [57]. A significance level of 5\% is chosen for this ANOVA study. Hence, the most significant factors have p-values less than 0.05 . The p-value for each term is used to test the null hypothesis that the coefficient equals zero, meaning no effect is existed [56]. For instance, considering the initial stiffness response characteristic, the significance level of the factors is determined based on their p-values. The p-value for the fuse end-width $(C)$, thickness $(D)$, and length $(A)$ is less than 0.0001 , which indicates the significance of their influence on the initial stiffness response.

In order to ensure the high accuracy of the statistical model, R-Squared, adjusted RSquared, predicted R-Squared, and adequate precision are checked. R-Squared value is used to determine the percentage of the data that can be explained by the model. An R-Squared value of at least 0.80 is typically desired for a good model. The adjusted R-Squared and predicted RSquared are used to define the relation of experimental data to their predicted values of the final equation. More specifically the predicted R-squared shows how well the regression model predicts responses for new observations. The difference between the adjusted R-Squared and the predicted R-Squared should not be greater than 0.20. Adequate precision measures the signal to noise ratio and a value of greater than 4 is desirable [56]. For example, considering the initial stiffness response, the value of R-Squared, the difference of adjusted and predicted R-Squared, and the adequate precision are $0.93,0.05$, and 17.84 , respectively.

Percentage contributions of important factors and interactions for each response characteristic are presented in Table 4.4. The positive sign indicates that the corresponding factor or interaction has a positive effect. As observed in Table 4.4, the overall behavior of steel plate fuses is controlled with the end-width and thickness while length and mid-width are less influential on some of the response variables. 
Table 4. 4 Percentage Contribution of important factors and interactions for each response characteristic.

\begin{tabular}{|c|c|c|c|c|c|c|c|c|c|c|c|}
\hline \multicolumn{12}{|c|}{ Response variable } \\
\hline \multicolumn{2}{|c|}{ Initial Stiffness } & \multicolumn{2}{|c|}{ Yield Strength } & \multicolumn{2}{|c|}{ Ultimate Stiffness } & \multicolumn{2}{|c|}{ Effective Damping } & \multicolumn{2}{|c|}{ Peak Strength } & \multicolumn{2}{|r|}{ Ductility } \\
\hline & Contribution & & Contribution & & Contribution & & Contribution & & Contribution & & Contribution \\
\hline Factor & $\%$ & Factor & $\%$ & Factor & $\%$ & Factor & $\%$ & Factor & $\%$ & Factor & $\%$ \\
\hline C & 22 & C & 29 & C & 24 & $D$ & 29 & $D$ & 43 & $D$ & 30 \\
\hline$D$ & 15 & $D$ & 29 & $D$ & 20 & C & -12 & C & 23 & $A$ & -26 \\
\hline$A$ & -15 & $C D$ & 18 & $A D$ & -8 & $C D$ & -11 & $C D$ & 16 & c & -9 \\
\hline$C D$ & 13 & $B$ & 5 & $A$ & -7 & $B$ & 8 & $B$ & 4 & C & 6 \\
\hline$A C$ & -12 & $B C$ & 5 & $C D$ & 6 & $B D$ & 7 & $B D$ & 3 & $A D$ & -5 \\
\hline$A D$ & -9 & $A$ & -2 & $c$ & 5 & $F_{y}$ & 5 & $A$ & -3 & $B C$ & -3 \\
\hline$B E$ & -7 & & & $A c$ & -4 & $D F_{y}$ & 5 & $A D$ & -2 & $A c$ & 3 \\
\hline & & & & $B E$ & -3 & $C F_{y}$ & -4 & & & $A E$ & -3 \\
\hline
\end{tabular}

\subsection{Sensitivity of Response Characteristics}

\subsubsection{Initial Stiffness}

In this section, the sensitivity of the initial stiffness response of steel plate fuses is discussed. As shown in Table 4.4, the most important factors considering the initial stiffness are the end-width $(C)$, thickness $(D)$, length $(A)$, as well as the interaction between end-width and thickness $(C D)$, length and end-width $(A C)$, length and thickness $(A D)$, and mid-width and modulus of elasticity $(B E)$ with the percentage contributions of $22 \%, 15 \%, 15 \%, 13 \%, 12 \%, 9 \%$ and $7 \%$, respectively. The remaining factors shown in Table 4.4 contribute less to the initial stiffness.

Among the significant factors, the end-width $(C)$, thickness $(D)$ and their interaction $(C D)$ show positive effects (i.e., the initial stiffness response is directly proportional to these factors). For example, by increasing the thickness from the low level $(5 \mathrm{~mm})$ to the high level $(40 \mathrm{~mm})$, the initial stiffness of the fuse is increased. On the other hand, the length $(A)$ and its interaction with the thickness $(A D)$, its interaction with the end-width $(A C)$, and the interaction between the midwidth and modulus of elasticity have negative effects on the initial stiffness $(B E)$. This observation is also supported by the results of previous research $[13,35]$ reporting that the initial stiffness of the fuse is directly proportional to the end-width and thickness and it is inversely proportional to the length. The formula for calculating the initial stiffness is as follows:

$$
K=\frac{2 E b t^{3}}{3 L^{3}}
$$


It is observed that, the most significant factors influencing the initial stiffness are all present in previously derived design formula (Eq. 3). The equation further supports the positive effect of end-width $(C)$, and thickness $(D)$ as well as the negative effect of length $(A)$, indicating that the initial stiffness of the fuse is directly proportional to the end-width and thickness and it is inversely proportional to the length.

The 3D plot in Figure 4.2 shows the effect of interaction $(C D)$ on the initial stiffness. It is observed that the effect of the thickness $(D)$ on the initial stiffness is significantly increased as the end-width $(C)$ is increased. As the thickness $(D)$ is increased (from $D=5 \mathrm{~mm}$ to $40 \mathrm{~mm}$ ) at the lower level of the end-width $(C=36 \mathrm{~mm})$, there is a slight change in the initial stiffness of the plate (approximately from 0 to $10 \mathrm{kN} / \mathrm{mm})$. However, at the high level of the end-width $(C=200$ $\mathrm{mm}$ ), with the increase in the thickness (from $D=5 \mathrm{~mm}$ to $D=40 \mathrm{~mm}$ ), the initial stiffness is significantly increased from 0 to approximately $400 \mathrm{kN} / \mathrm{mm}$.

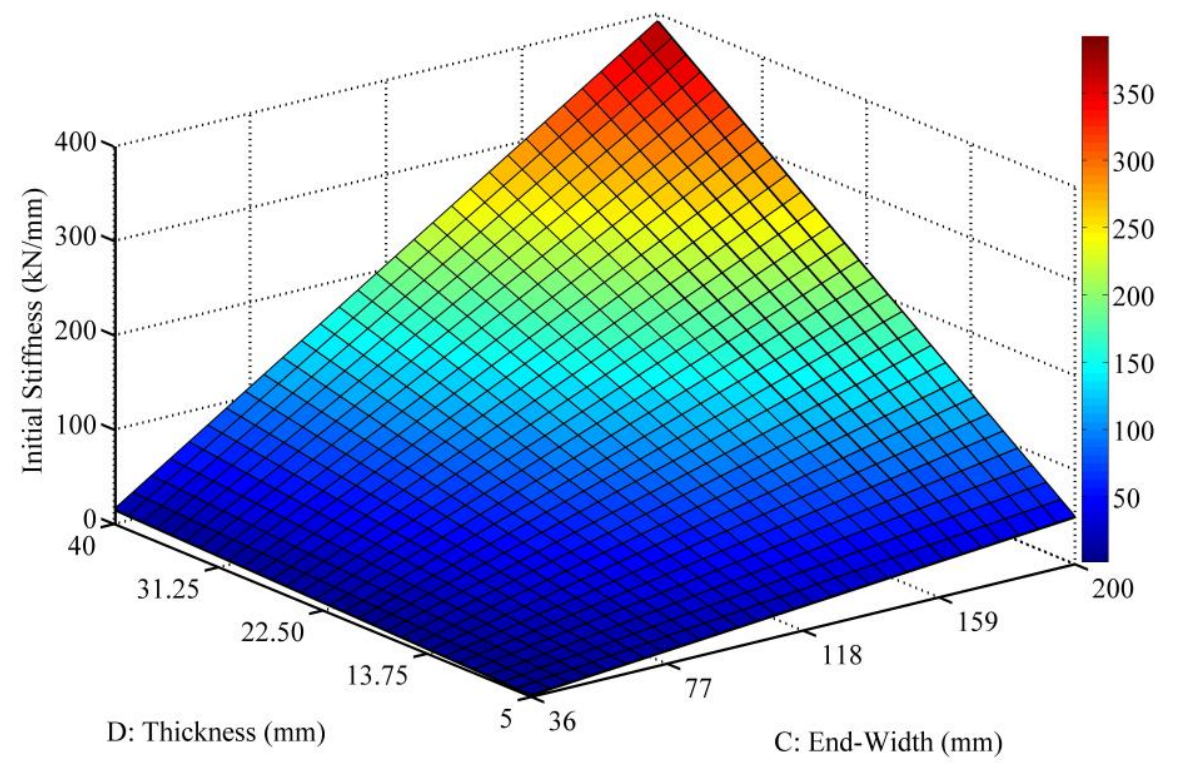

Figure 4. 2 The effect of interaction between the thickness and the end-width on initial stiffness.

\subsubsection{Yield Strength}

Another response value that is studied from the results of sensitivity analysis is the yield strength of the steel plates. The yield strength is a significant response characteristic in the steel plate fuses since the fuse should have enough yielding capacity to stay undamaged under service 
loads [15]. The sensitivity analysis results show that the yield strength of steel plate fuses is mostly affected by the end-width $(C)$, thickness $(D)$, mid-width $(B)$, length $(A)$, as well as the interactions $C D$ and $B C$ having percentage contributions of 29\%, 29\%, 5\%,2\%, 18\%, and $5 \%$ respectively (as shown in Table 4.4. Among all the main factors, only the fuse length $(A)$ has a negative impact on the yield strength. In addition, it can be observed that over $75 \%$ of the variability in the fuse yield strength is controlled by the end-width $(C)$, thickness $(D)$ and their interaction $(C D)$. The analysis results are additionally confirmed by the yield strength design formula (Eq. 4) [13,51].

$$
P_{y}=\frac{n b^{2} t F_{y}}{3 L}
$$

It is perceived that the factors influencing the yield strength are all present in the previously derived design formula (Eq. 4). It should be noted that Eq. 4 is derived only for straight (slit) fuses excluding the effect of mid-width $(B)$, which is included in the present sensitivity study. The equation further confirms the positive effect of end-width $(C)$ and thickness $(D)$ as well as the negative effect of length $(A)$ - showing that the yield strength of the fuse is directly proportional to the end-width and thickness and it is inversely proportional to the length.

The 3D plot in Figure 4.3 shows the interaction between the end-width $(C)$ and the thickness $(D)$ of the steel plate fuse. It can be observed that increasing both the end-width $(C)$ and the thickness $(D)$ from their low levels $(D=5 \mathrm{~mm}, C=36 \mathrm{~mm})$ to high levels $(D=40 \mathrm{~mm}, C=200$ $\mathrm{mm}$ ) increases the yield strength from 0 to approximately $165 \mathrm{kN}$. 


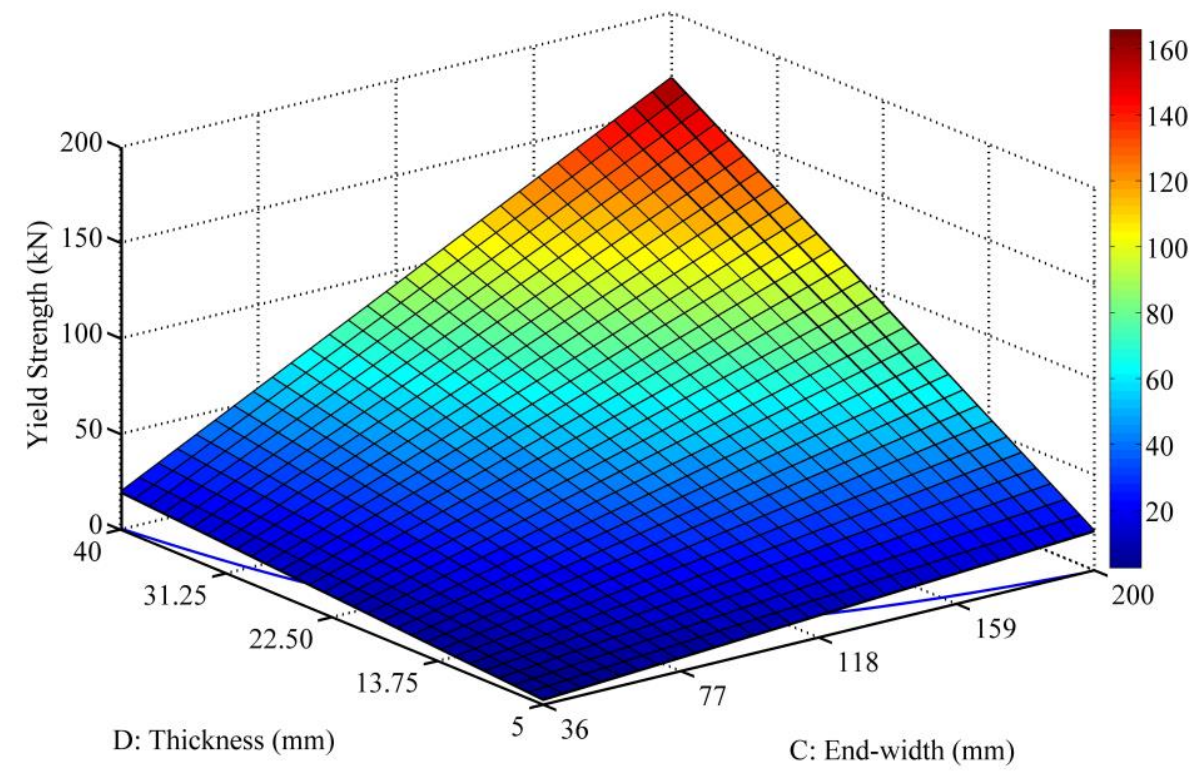

Figure 4. 3 The effect of interaction between the end-width and the thickness on yield strength.

\subsubsection{Ultimate Stiffness}

The ultimate stiffness of steel plate fuses is mostly affected by end-width $(C)$, thickness $(D)$, length $(A)$, and the interaction between length and thickness $(A D)$. This observation is likewise the sensitivity results for the maximum and yield strength, which were found sensitive to $C, D$, and $A$. The calculated percentage contributions for the main factors and interactions indicate that these effects (i.e. the effect of $C, D, A, c, A D, C D, A c$, and $B E$ ) account for $24 \%, 20 \%, 7 \%$, $5 \%, 8 \%, 6 \%, 4 \%$ and $3 \%$ of the total response variability, respectively. As presented in Table 4.4 end-width $(C)$, thickness $(D)$ band zone $(c)$, and interaction $(C D)$ have positive effects while the remaining factors have negative effects.

To determine the effect of interaction between the main factors length $(A)$ and thickness $(D)$ on the variability of the ultimate stiffness response, the 3D plot in Figure 4.4 is illustrated. It can be observed that the ultimate stiffness response increases approximately from 0.40 to $2.25 \mathrm{kN}$ as the thickness $(D)$ increases from its low level $(D=5 \mathrm{~mm})$ to high level $(D=40 \mathrm{~mm})$ and the length $(A)$ decreases from its high level $(A=500 \mathrm{~mm})$ to its low level $(A=180 \mathrm{~mm})$. 


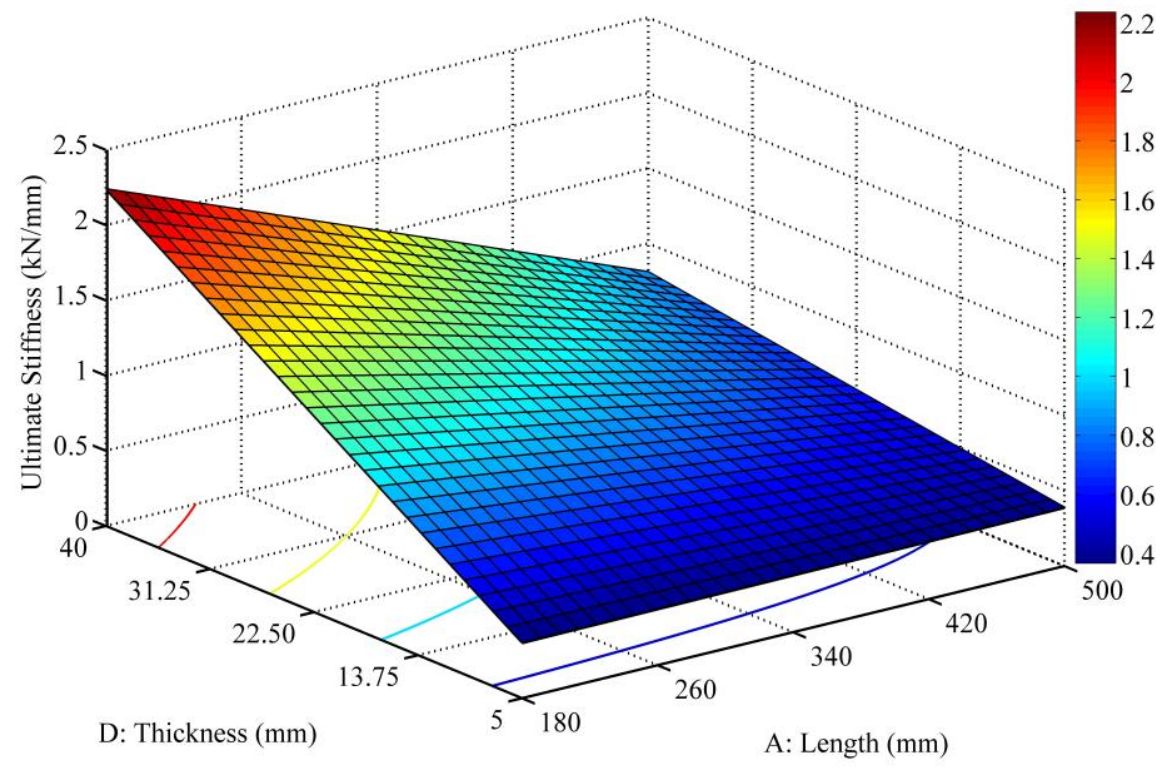

Figure 4. 4 The effect of interaction between the length and the thickness on ultimate stiffness.

\subsubsection{Effective Damping}

This section describes the sensitivity analysis of the effective damping response. Factors that have a significant effect on the effective damping are the thickness $(D)$, end-width $(C)$, midwidth $(B)$, steel yield strength $\left(F_{y}\right)$, the two-factor interactions between end-width and thickness $(C D)$, between mid-width and thickness $(B D)$, between thickness and steel yield strength $\left(D F_{y}\right)$, and between end-width and steel yield strength $\left(C F_{y}\right)$. The remaining factors such as the length $(A)$, modulus of elasticity $(E)$ and the band zone length $(c)$ have shown no significant effect on the effective damping response characteristics.

From the significant factors, the thickness $(D)$, mid-width $(B)$, steel yield strength $\left(F_{y}\right)$, the interactions between mid-width and thickness $(B D)$, thickness and steel yield strength $\left(D F_{y}\right)$ have positive effects with the percentage contributions of $29 \%, 8 \%, 5 \%, 7 \%$, and $5 \%$, respectively (as listed in Table 4.4). On the other hand, the end-width $(C)$, the interactions between the end-width and thickness $(C D)$, and between the end-width and modulus of elasticity $(C E)$ show negative effects on the effective damping with percentage contributions of $12 \%, 11 \%$, and $4 \%$, respectively.

These results indicate that the effective damping of steel plate fuses is mainly controlled by the thickness, end-width, and their interaction having a combined contribution of more than 
50\%. This trend can be attributed to the fact that the energy dissipating capacity of steel plate fuse is increased significantly with an increase of the thickness because of the delay in the buckling of the fuses. This is further supported by Farzampour and Eatherton [40], suggesting that the thickness of the plate is observed to control the buckling limit state of the fuse, and thus affecting the energy dissipation capability. Further, the negative effect of the end-width on the effective damping is expected because increasing the end-width over the length causes the fuse to act more like a solid plate thus leading to less energy dissipation. These observations are also consistent with the research by Farzampour and Eatherton [40]. They reported that increasing the end width over the length of the butterfly-shaped fuses would make the shear mechanism governing over the flexure, and thus resulting in a reduction in the energy dissipation [40].

To examine the effect of the interaction between the main factors end-width $(C)$ and thickness $(D)$ on the variability of the effective damping of steel fuse plates, the 3D plot in Figure 4.5 is presented. It can be observed that at low level of end-width $(C=36 \mathrm{~mm})$, increasing the thickness $(D)$ significantly increases the effective damping response (1 to 215000). In contrast, at high level of end-width ( $C=200$ ), the effective damping is increased at a lesser rate (from 1 to $5000)$ due to the increase in the thickness $(D)$. This trend is because of the active interaction between $C$ and $D$. In other words, since the interaction is involved, the amount of change in the response because of varying thickness $(D)$ is different at different levels of the end-width $(C)$. 


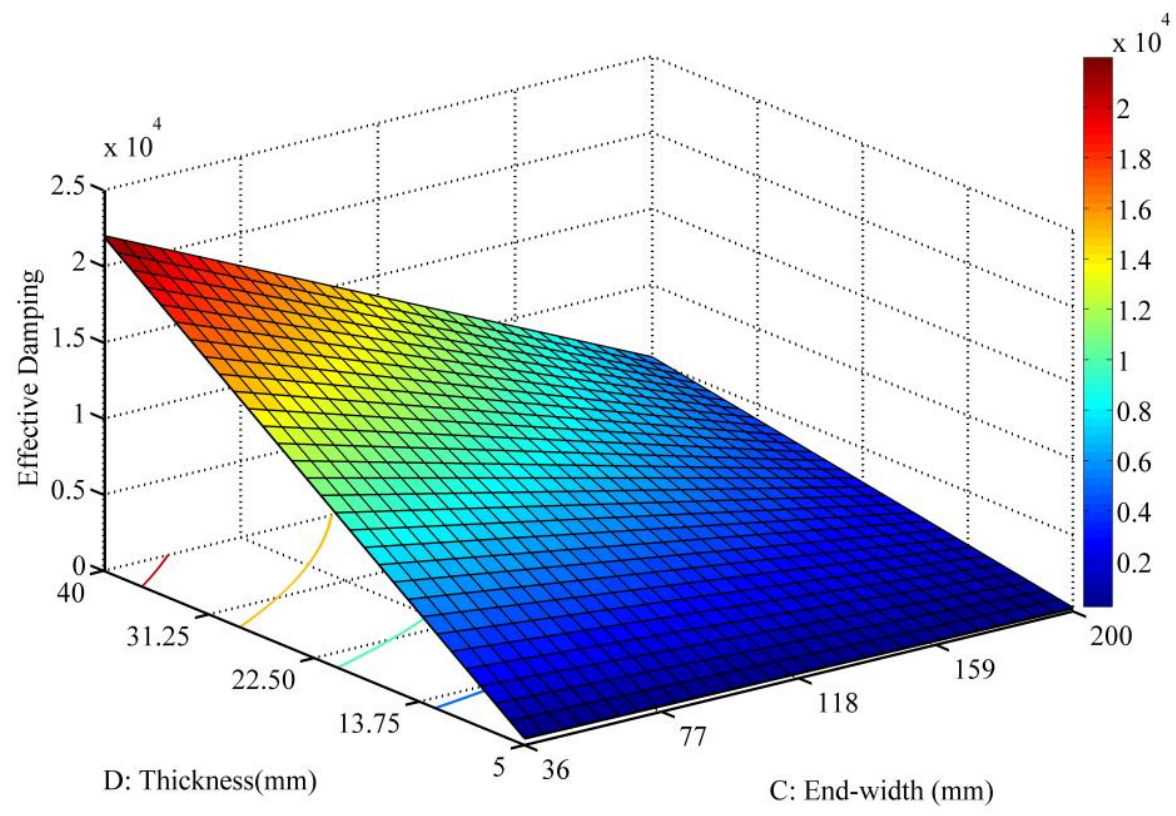

Figure 4. 5 The effect of interaction between the end-width and the thickness on effective damping.

\subsubsection{Maximum Strength}

The shear load capacity of steel plate fuses is monitored as a response characteristic. As shown in Table 4.4, the important factors that are influencing the load capacity of the fuses are the thickness $(D)$, end-width $(C)$, mid-width $(B)$, length $(A)$, the interaction between end-width and thickness $(C D)$, the interaction between length and thickness $(A D)$, and the interaction between mid-width and thickness $(B D)$ with percentage contributions of 43\%, 23\%, 4\%, 3\%, 16\%, 2\%, and $3 \%$, respectively.

The length $(A)$ and interaction $(A D)$ have negative effects while all the other significant factors and interactions have positive impacts on the load capacity. The end-width $(C)$, thickness $(D)$, and their interaction $(C D)$ account for over $80 \%$ contribution. The response sensitivity result can be explained based on the buckling behavior of steel fuses. Past experimental studies have confirmed that, because of buckling, thin and long steel plate fuses reach less strength compared to thick fuses. The experimental study by Lee et al. [15] reports that the load capacity of steel plate fuses is significantly decreased when the fuse experiences buckling under cyclic loading.

The response sensitivity results for the maximum strength are also supported by the previously developed design formula [13]: 


$$
P=\frac{n b^{2} t \mathrm{~F}_{y}}{2 L}
$$

It is confirmed that the factors influencing the maximum strength are all present in the previously derived design formula (Eq. 5). This equation however is derived only for straight (slit) fuses excluding the effect of mid-width $(B)$, which is included in this sensitivity analysis. This equation further confirms the positive effect of thickness $(D)$ and end-width $(C)$ as well as the negative effect of length $(A)$ - indicating that the maximum strength of the fuse is directly proportional to the end-width and thickness, and it is inversely proportional to the fuse length.

Among the significant interactions, the interaction between the end-width $(C)$ and the thickness $(D)$ is noticeable. The $C D$ interaction is illustrated in Figure 4.6. It can be observed that the maximum load response increases from 0 to $400 \mathrm{kN}$ as the end-width $(C)$ increases from its low level $(C=36 \mathrm{~mm})$ to high level $(C=200 \mathrm{~mm})$, when the thickness $(D)$ is kept at its high level ( $D=40 \mathrm{~mm})$. In contrast, at the low level of thickness $(D=5 \mathrm{~mm})$, the increase in the end-width $(C)$ from its low level $(C=36 \mathrm{~mm})$ to high level $(C=200 \mathrm{~mm})$ only increases the maximum load response from 1 to approximately $20 \mathrm{kN}$.

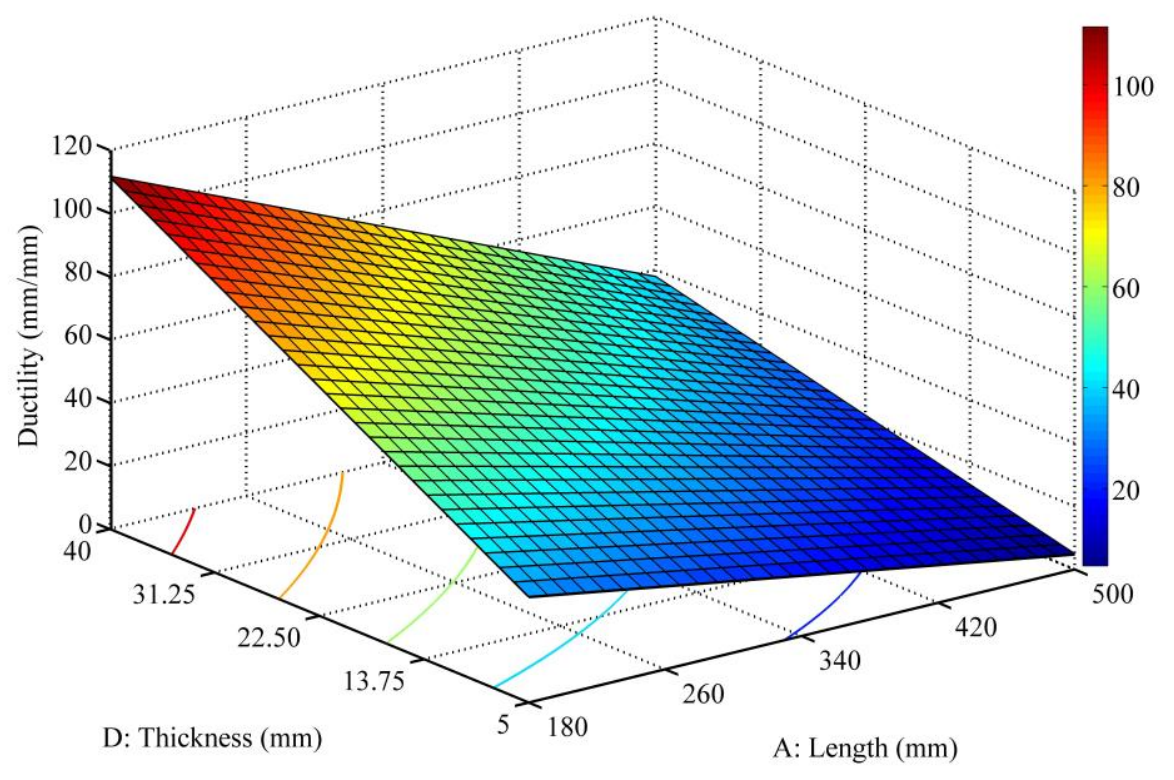

Figure 4. 6 The effect of interaction between the end-width and the thickness on maximum strength. 


\subsubsection{Ductility}

The ductility of steel plate fuses is mostly affected by the thickness $(D)$, length $(A)$, band zone length $(c)$, and end-width $(C)$ having percentage contributions of $30 \%, 26 \%, 9 \%$, and $6 \%$, respectively (Table 4.4). The length and the thickness of steel plate fuses are found to control over $50 \%$ of the ductility response. This fact can be expected because steel plate fuses with higher thickness and lower lengths are less prone to pinching and thus these fuses are expected to show large deformations. This fact is also confirmed by the experimental study by Ma et al. [13]. They reported that thick strip dampers (steel plate fuses with straight and butterfly-shaped openings) can undergo large deformations and can have an uncompromised performance since pinching is delayed in the damper [13]. In addition to thickness, the importance of length influencing the deformation capacity is also observed in the experimental study by Ma et al. in [13] reporting that by changing the fuse length, the deformation demand in terms of shear deformation can be adjusted to a desired value. As listed in Table 4.4, the ductility response also appears to be affected by interactions $(A D),(B C),(A C)$, and $(A E)$ with percentage contributions of $5 \%, 3 \%, 3 \%$, and $3 \%$, respectively.

Depending on the factor combination, a wide range of ductility response from 2.06 to 200.46 $\mathrm{mm} / \mathrm{mm}$ is observed. The 3D plot in Figure 4.7, illustrates how the $(A D)$ interaction influences the ductility response. As the thickness increases, for longer fuses, the ductility is increased from 3 to about $40 \mathrm{~mm} / \mathrm{mm}$ while for shorter fuses, the ductility is increased from 30 to $110 \mathrm{~mm} / \mathrm{mm}$ when the other factors are at their mean values. 


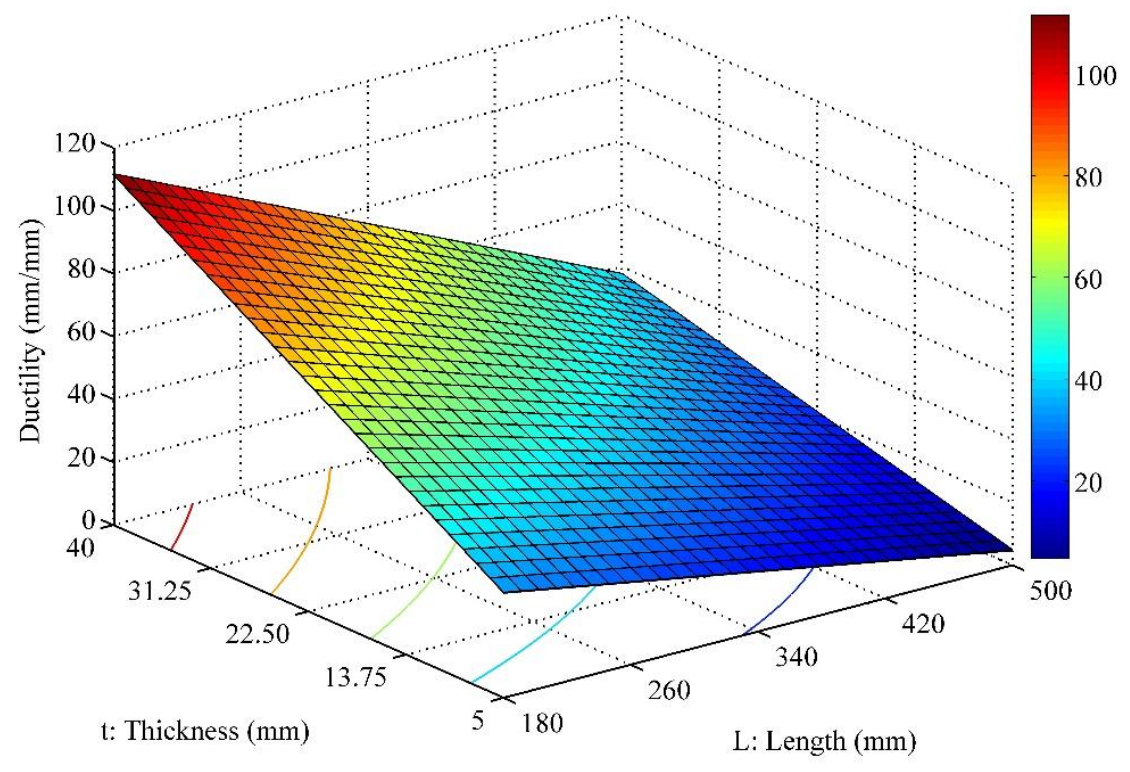

Figure 4. 7 The effect of interaction between the thickness and the length of the link on ductility. 


\section{CHAPTER 5}

\section{PREDICTIVE MODELS FOR ENERGY DISSIPATING STEEL PLATE FUSES}

\subsection{Overview}

From the potentially influential parameters identified in previous chapters, the ratios of four parameters are chosen for further study in this chapter. These factors, which are the ratios of the most influential parameters on the response characteristics include: the length to end-width, end-width to thickness and the mid-width to end-width ratios of the fuse link. The goal of this chapter is to derive predictive equations for the response characteristics described in previous chapters. A response surface methodology (RSM) is used to generate predictive equations for each response characteristic.

\subsection{Factors and Response Characteristics}

The ratios of four most influential design parameters are considered for the response surface study. While considering these ratios the value of end-width is kept constant to make all the ratios independent of each other. Table 5.1 lists these factors with their corresponding ranges, which are the most influential parameters on the cyclic response of steel plate fuses [58].

Table 5. 1 Factors and ranges considered for the response surface study.

\begin{tabular}{ccccc}
\hline Factors & Symbol & Low level (-) & High level (+) & Units \\
\hline Length to End-width & $L / b$ & 2 & 6 & - \\
End-width to Thickness & $b / t$ & 3 & 15 & - \\
Mid-width to End-width & $a / b$ & 0.15 & 1.00 & - \\
\hline
\end{tabular}

A central composite design (CCD) is used to generate factor combinations for the response surface study. The central composite design contains an imbedded factorial or fractional factorial design with center points that is augmented with a group of star points which permits the proper estimation of curvature for any desirable response [56]. Table 5.2 lists the factor combinations created using the Design-Expert software [56]. Each combination corresponds to a fuse model for which a nonlinear cyclic analysis is performed. 
Table 5. 2 Factor combinations for the simulation runs.

\begin{tabular}{cccc}
\hline Model & $L / b$ & $b / t$ & $a / b$ \\
\hline 1 & 2.00 & 3.00 & 0.15 \\
2 & 6.00 & 9.00 & 0.58 \\
3 & 2.00 & 3.00 & 1.00 \\
4 & 6.00 & 3.00 & 0.15 \\
5 & 2.00 & 15.0 & 1.00 \\
6 & 6.00 & 15.0 & 0.15 \\
7 & 4.00 & 9.00 & 1.00 \\
8 & 4.00 & 15.0 & 0.58 \\
9 & 6.00 & 15.0 & 1.00 \\
10 & 4.00 & 3.00 & 0.58 \\
11 & 6.00 & 3.00 & 1.00 \\
12 & 4.00 & 9.00 & 0.15 \\
13 & 4.00 & 9.00 & 0.58 \\
14 & 2.00 & 9.00 & 0.58 \\
15 & 2.00 & 15.0 & 0.15 \\
\hline
\end{tabular}

Five response characteristics which include the initial stiffness, yield strength, effective damping, peak strength, and ductility are recorded for the newly generated FE models (Table 5.2). The calculation procedure of each response characteristic is explained in previous chapters in a detailed manner.

\subsection{Results of the Cyclic Response Simulations}

The cyclic load-displacement response of each model is obtained from the nonlinear analysis of the fuse model. A wide range of cyclic response is observed. For example, the cyclic response plots for four models 2, 4, 8 and 13 are illustrated in Figure 5.1. Model 8 with the factor combinations shown in Table 5.2 has shown greater ductility compared to models 2, 4, and 13. The effective damping in model 2 is $1.50,1.80$, and 1.45 times higher than that in models 4,8 , and 13; respectively. The maximum strength and yield strength response are found to be highest in model 4 while model 13 has the highest initial stiffness among these four models. A list of response 
characteristics computed from each simulation run is provided in Table 5.3. In these models, models 2 and 4 are considered the best models in terms of seismic performance because they provide greater ductility and dissipate more energy when compared to other models.

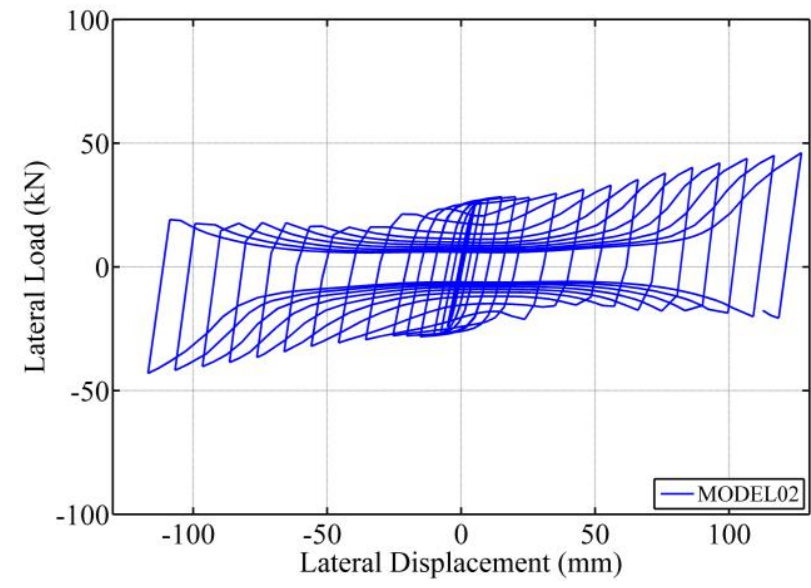

(a)

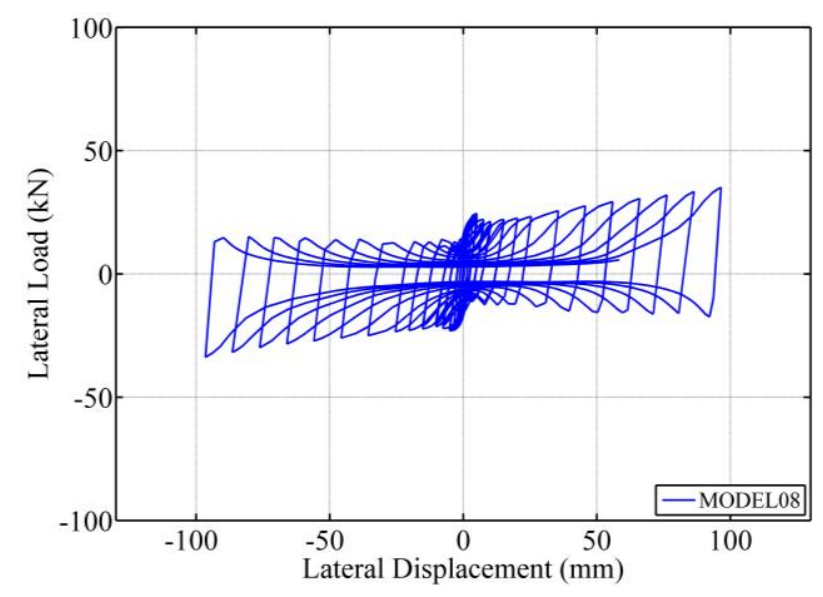

(c)

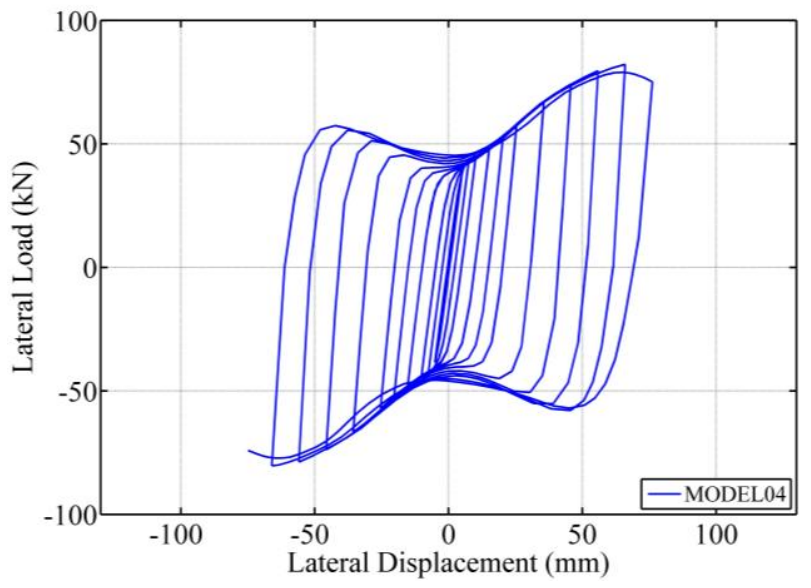

(b)

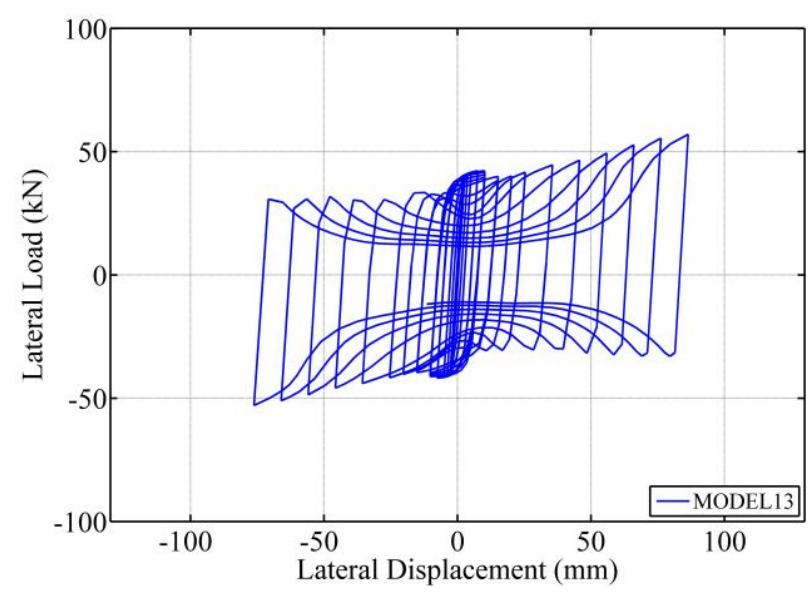

(d)

Figure 5. 1 Hysteretic response plots for: (a) model 2, (b) model 4, (c) model 8, and (d) model 13.

\subsection{Results and Predictive Equations}

Based on least-square regression analyses performed in Design-Expert [56], equations are developed to quantitatively express a relationship between the response variable and the input factors. Figures 5.2 to 5.7 illustrate how each response characteristic is changed with respect to pairs of input factors (all other input factors are set to their center points). For instance, as 
illustrated in Figure 5.2, the initial stiffness response is highest for smaller length to end-width and end-width to thickness ratios and larger mid-width to end-width ratios. The effect of length to endwidth ratio is greater at low levels of end-width to thickness ratios, whereas, at high levels of length to end-width the effect of length to end-width is insignificant. This observation is because of the active interaction between the two ratios.

Table 5. 3 Response characteristics of each model.

\begin{tabular}{cccccc}
\hline Model & $\begin{array}{c}\text { Initial } \\
\text { Stiffness }\end{array}$ & $\begin{array}{c}\text { Yield } \\
\text { Strength }\end{array}$ & $\begin{array}{c}\text { Effective } \\
\text { Damping }\end{array}$ & $\begin{array}{c}\text { Maximum } \\
\text { Strength }\end{array}$ & Ductility \\
& $\mathrm{kN} / \mathrm{mm}$ & $\mathrm{kN}$ & & $\mathrm{kN}$ & $\mathrm{mm} / \mathrm{mm}$ \\
\hline 1 & 155.10 & 76.0 & 321.078 & 138.9 & 15.51 \\
2 & 5.4485 & 16.4 & 5155.68 & 46.08 & 42.19 \\
3 & 333.33 & 150 & 319.081 & 285.2 & 17.47 \\
4 & 8.0526 & 30.6 & 3458.53 & 82.16 & 20.05 \\
5 & 63.469 & 31.1 & 382.803 & 51.71 & 15.51 \\
6 & 1.5394 & 5.85 & 2149.04 & 12.50 & 20.05 \\
7 & 22.452 & 23.8 & 995.308 & 45.42 & 15.20 \\
8 & 9.6000 & 14.4 & 2870.86 & 35.11 & 64.33 \\
9 & 4.4400 & 11.1 & 3259.40 & 28.13 & 46.72 \\
10 & 50.600 & 75.9 & 4693.12 & 216.1 & 66.46 \\
11 & 23.120 & 57.8 & 5517.68 & 146.8 & 31.85 \\
12 & 8.3333 & 12.5 & 912.497 & 27.80 & 14.51 \\
13 & 16.600 & 24.9 & 3542.71 & 57.02 & 45.47 \\
14 & 89.833 & 53.9 & 904.824 & 82.23 & 25.33 \\
15 & 27.755 & 13.6 & 69.0271 & 20.90 & 7.760 \\
\hline
\end{tabular}

The response surface models developed in this section are predictive equations that quantitatively estimate the relationship between the response characteristic variable, $y$, (e.g. initial stiffness, $K$ ) and the input design factors, $x_{i}$ (e.g. length to end-width ratio, $L / b$ ). A quadratic polynomial relationship [Eq. (3)] is adopted to relate each response to the design factors. The 
reason behind using the quadratic polynomial equation is to capture the curvature of each response over the ranges of each design parameter.

$y=\beta_{0}+\sum_{i=1}^{k} \beta_{i} x_{i}+\sum_{i=1}^{k} \sum_{j=1}^{k} \beta_{i j} x_{i} x_{j}+\varepsilon \quad$ Eq. (3)

In this equation, the $\beta$ 's are the unknown regression coefficients and $\varepsilon$ is a random error term (residual) that shows the difference between the actual $\left(y_{l}\right)$ and predicted response values $\left(\widehat{y}_{l}\right)$, i.e. $\varepsilon_{l}=y_{l}-\widehat{y}_{l}$ for each factor combination or design point $(l=1,2, \ldots, n)$. The least squares method is used to determine the regression coefficients, where the $\beta$ 's minimize the sum of the squares of the errors $\left(\sum_{l=1}^{n} \varepsilon_{l}^{2}\right)$ [34]. Tables 5.4 to 5.10 provide the regression coefficients $\beta_{i}$ and $\beta_{i j}$ corresponding to each response variable. The response surface model is a quadratic function of the actual values of each factor (not the coded values of $-1,+1$ ). The following sections present the results for each response variable.

Table 5. $4 \beta_{i}$ values for all the response variables.

\begin{tabular}{ccccccc}
\hline$i$ & Input parameter, $x_{i}$ & \multicolumn{5}{c}{$\beta_{i}$ 's for each response variable } \\
\cline { 2 - 6 } & & $K$ & $P_{y}$ & $\beta_{\text {eff }}$ & $P$ & $\mu$ \\
\hline & Transformation & $\ln (y)$ & $\ln (y)$ & $\sqrt{y}$ & $\ln (y)$ \\
\hline 0 & intercept & 7.869 & 5.838 & -21.15 & 6.044 & -15.21 \\
1 & $L / b$ & -1.322 & -0.644 & 16.47 & -0.313 & 23.72 \\
2 & $b / t$ & -0.285 & -0.299 & -3.960 & -0.383 & -11.25 \\
3 & $a / b$ & 2.007 & 3.010 & 141.0 & 3.695 & 183.8
\end{tabular}

* Note: All the response variables are transformed except the ductility, where $y$ is considered as the response. Ductility response variable shows higher accuracy without any transformation in the response.

\subsubsection{Initial Stiffness}

The initial stiffness of steel plate fuses ranges from $1.54 \mathrm{kN} / \mathrm{mm}$ to $333.33 \mathrm{kN} / \mathrm{mm}$. The general relationship that is presented in Eq. (3) with the regression coefficients recorded in Table 5.4 and 5.5 can be used to predict the initial stiffness of steel plate fuses with the input parameters ranging from their low levels to high levels (in Table 5.1). Analysis of variance (ANOVA) with a significance level of $5 \%$ is used to fit a quadratic model for the natural log-transformed response as listed in Tables 5.4 to 5.10. Box-Cox diagnostics method is used to find the best fit 
transformation for the response variable. ANOVA presents adjusted $R^{2}$ value of 0.999 . Higher values of adjusted $R^{2}$ demonstrate that the response variable is predicted well over the design space [56]. The variation of the initial stiffness response to pairs of input parameters are shown in Figure 5.2 .

Table $5.5 \beta_{i j}$ 's for initial stiffness.

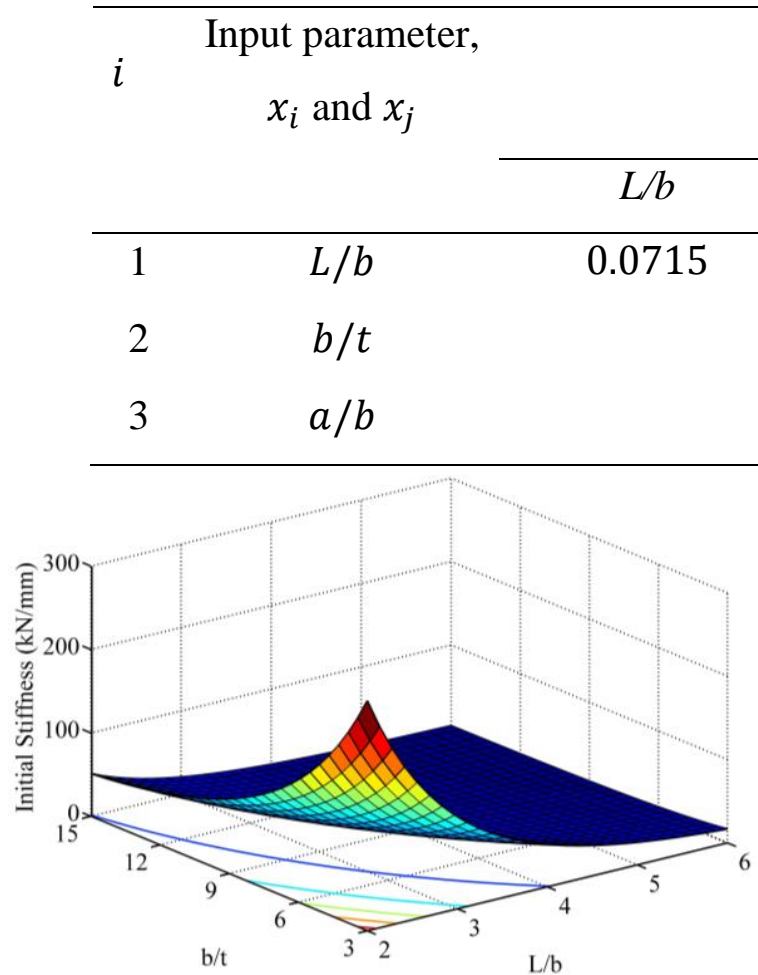

(a)

$$
\beta_{i j} \text { 's for } \ln (y)
$$

$\begin{array}{cc}b / t & a / b \\ 7.8 \times 10^{-4} & 0.077 \\ .8 \times 10^{-3} & 3.3 \times 10^{-3} \\ & -1.076\end{array}$

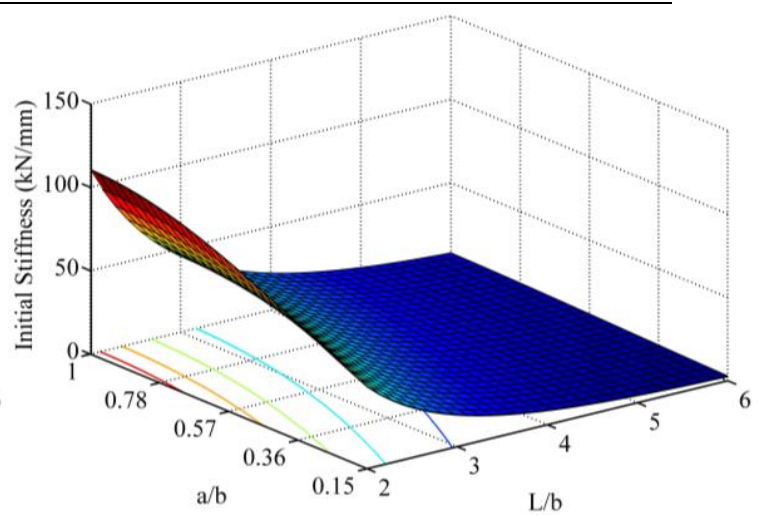

(b)

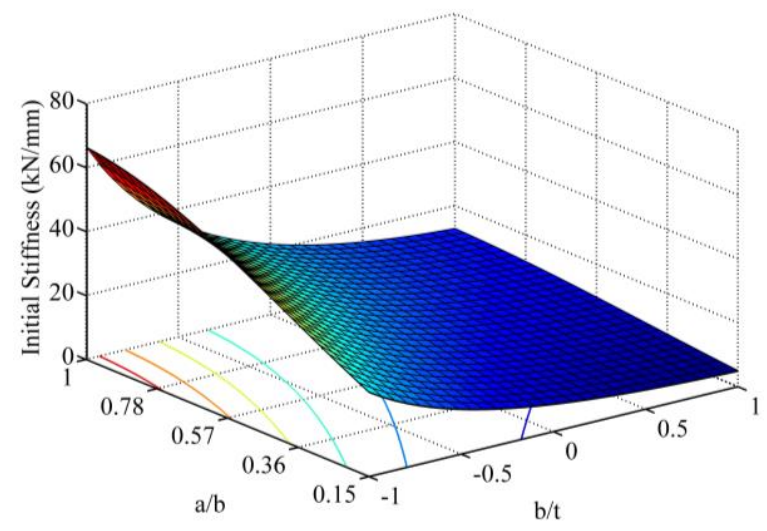

(c)

Figure 5. 2 Variation of initial stiffness with respect to different pairs of design factors. 


\subsubsection{Yield Strength}

The simulation results show that the yield strength of steel plate fuses is changing from $5.85 \mathrm{kN}$ to $150 \mathrm{kN}$. A quadratic model is used to fit the data with the natural log-transformed response using Box-Cox diagnostic method. ANOVA indicates adjusted $R^{2}$ value of 0.992. Eq. (3) with the values of regression coefficients presented in Tables 5.4 and 5.6 can be used to accurately predict the yield strength of steel plate fuses. The variation of the yield strength response with respect to pairs of input parameters are shown in Figure 5.3.

Table 5. $6 \beta_{i j}$ 's for yield strength.

\begin{tabular}{|c|c|c|c|c|}
\hline \multirow[t]{2}{*}{$i$} & \multirow{2}{*}{$\begin{array}{c}\text { Input parameter, } \\
x_{i} \text { and } x_{j}\end{array}$} & \multicolumn{3}{|c|}{$\beta_{i j}$ 's for $\ln (y)$} \\
\hline & & $L / b$ & $b / t$ & $a / b$ \\
\hline 1 & $L / b$ & 0.052 & $-1.1 \times 10^{-4}$ & -0.034 \\
\hline 2 & $b / t$ & & $8.8 \times 10^{-3}$ & $7.4 \times 10^{-3}$ \\
\hline 3 & $a / b$ & & & -1.857 \\
\hline
\end{tabular}




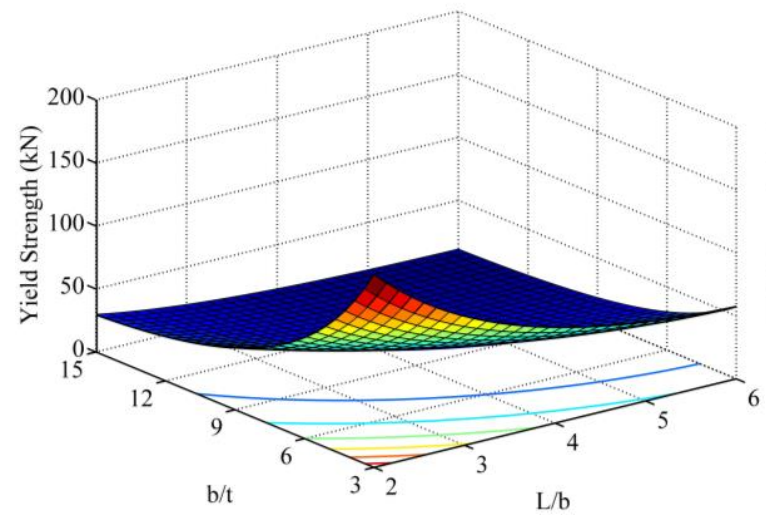

(a)

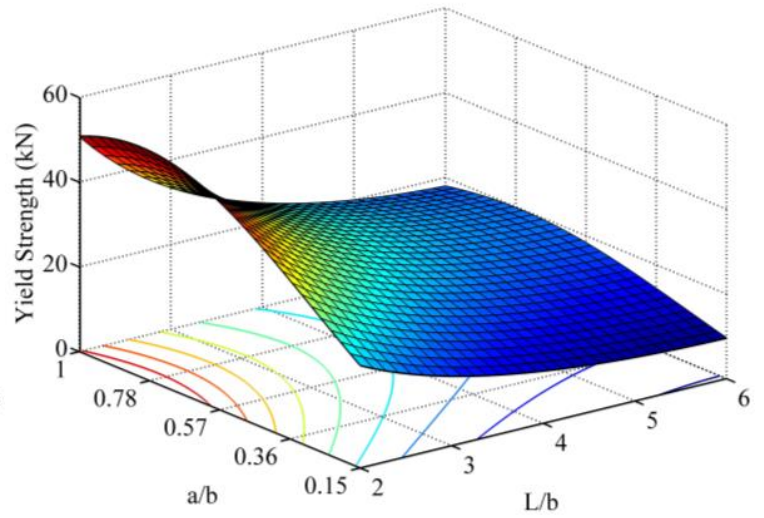

(b)

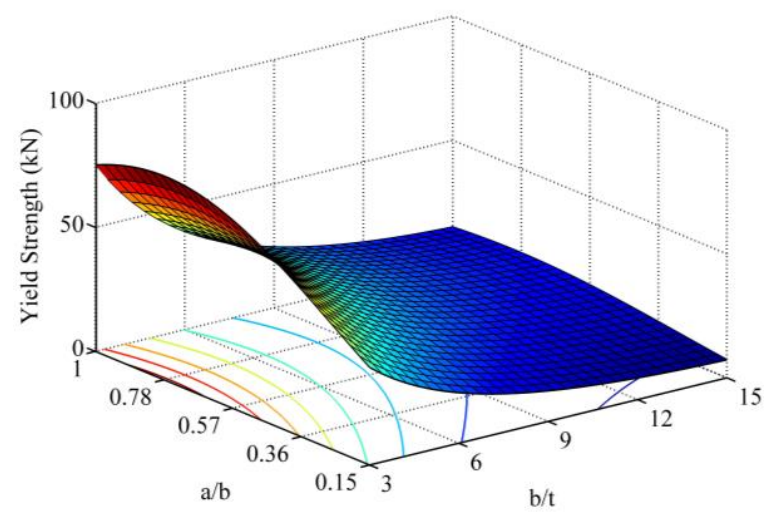

(c)

Figure 5. 3 Variation of yield strength with respect to different pairs of design parameters.

\subsubsection{Effective Damping}

The effective damping of steel plate fuses ranges from 69.03 to 5517.68. The response surface predictive equation is a quadratic function for the effective damping response with a squared root transformation. ANOVA indicates adjusted $R^{2}$ value of 0.957 for this response variable. Eq. (3) with the regression coefficients provided in Tables 5.4 and 5.8 can be used to accurately predict the effective damping of steel plate fuses with the input parameters ranging from their low levels to high levels (table 2). The 3D plots in Figure 5.5 illustrate the variation of the response with respect to pairs of input parameters. 
Table $5.7 \beta_{i j}$ 's for effective damping.

\begin{tabular}{ccccc}
\hline \multirow{3}{*}{$i$} & $\begin{array}{c}\text { Input parameter, } \\
x_{i} \text { and } x_{j}\end{array}$ & \multicolumn{3}{c}{$\beta_{i j}$ 's for $\sqrt{y}$} \\
\cline { 3 - 5 } & & $L / b$ & $b / t$ & $a / b$ \\
\hline 1 & $L / b$ & -0.622 & -0.227 & 2.206 \\
2 & $b / t$ & & 0.211 & 0.322 \\
3 & $a / b$ & & -124.9 \\
\hline
\end{tabular}
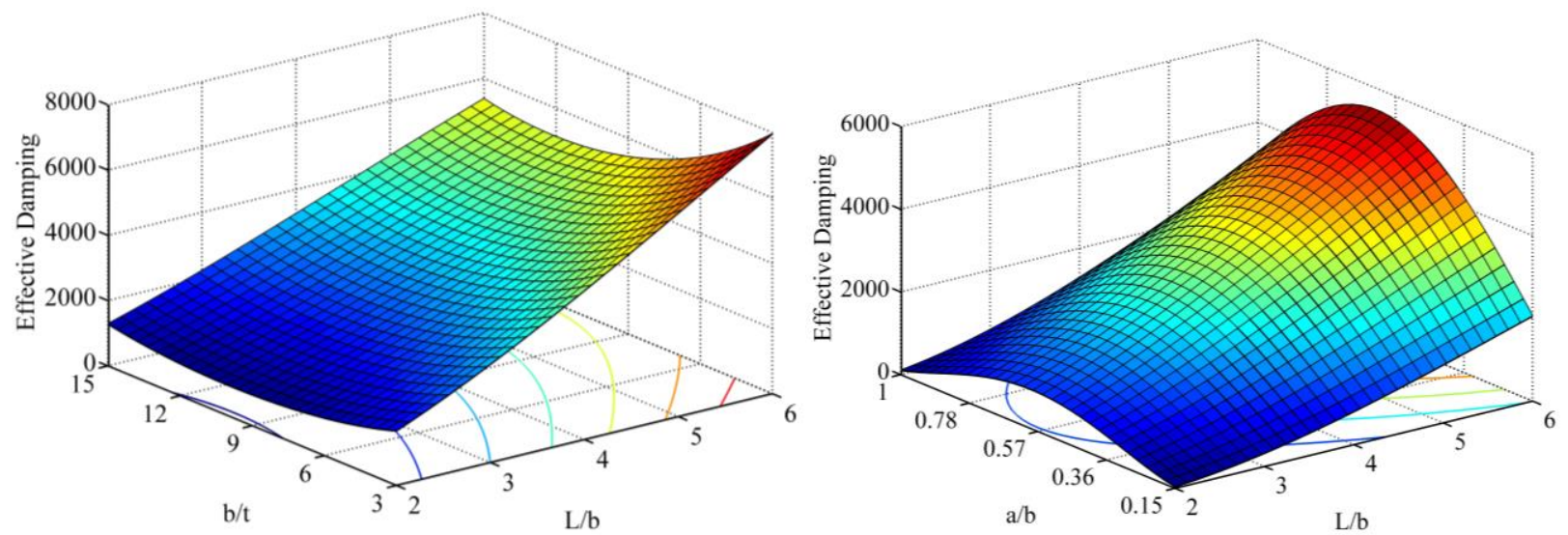

(a)

(b)

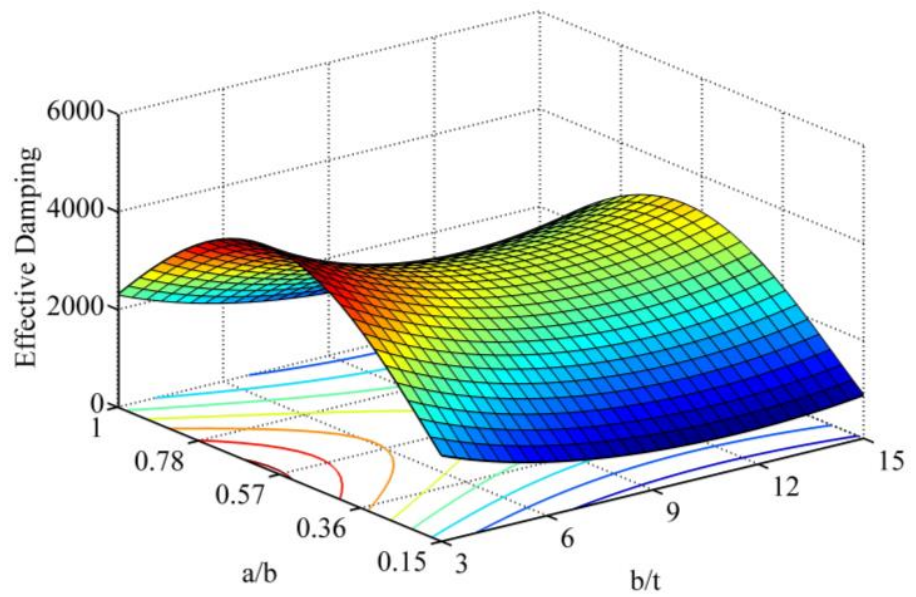

(c)

Figure 5. 4 Variation of effective damping with respect to different pairs of design factors. 


\subsubsection{Maximum Strength}

The results show that the maximum strength of steel plate fuses is changing from $12.51 \mathrm{kN}$ to $285.27 \mathrm{kN}$. A quadratic model is used to fit the data with the natural log-transformed response. Tables 5.4 and 5.9 provide the regression coefficients. The adjusted $R^{2}$ value is 0.993 , respectively. These values indicate that the developed equation can accurately predict the maximum strength of steel plate fuses. Figure 5.6 is used to illustrate the variation of maximum strength response to pairs of input parameters.

Table $5.8 \beta_{i j}$ 's for maximum strength.

\begin{tabular}{ccccc}
\hline \multirow{3}{*}{$\begin{array}{c}\text { Input parameter, } \\
x_{i} \text { and } x_{j}\end{array}$} & \multicolumn{3}{c}{$\beta_{i j}$ 's for $\ln (y)$} \\
\cline { 3 - 5 } & & $L / b$ & $b / t$ & $a / b$ \\
\hline 1 & $L / b$ & 0.023 & $7.1 \times 10^{-4}$ & -0.034 \\
2 & $b / t$ & & 0.012 & 0.020 \\
3 & $a / b$ & & -2.536 \\
\hline
\end{tabular}




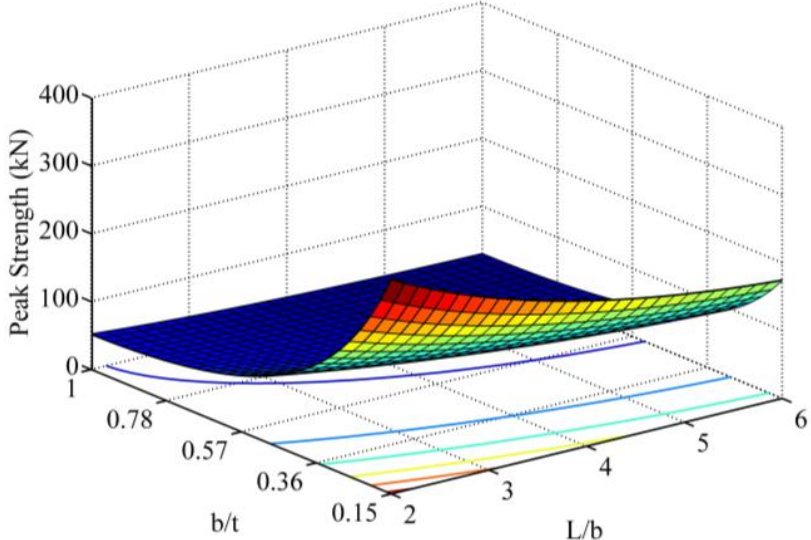

(a)

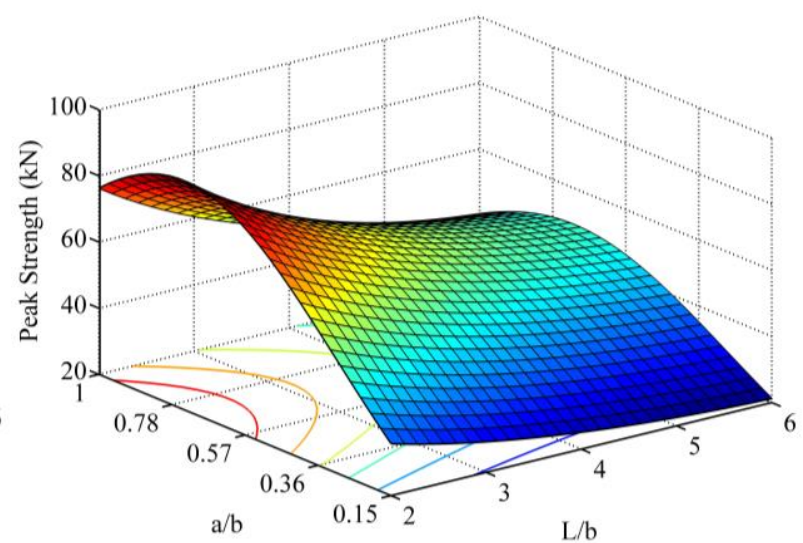

(b)

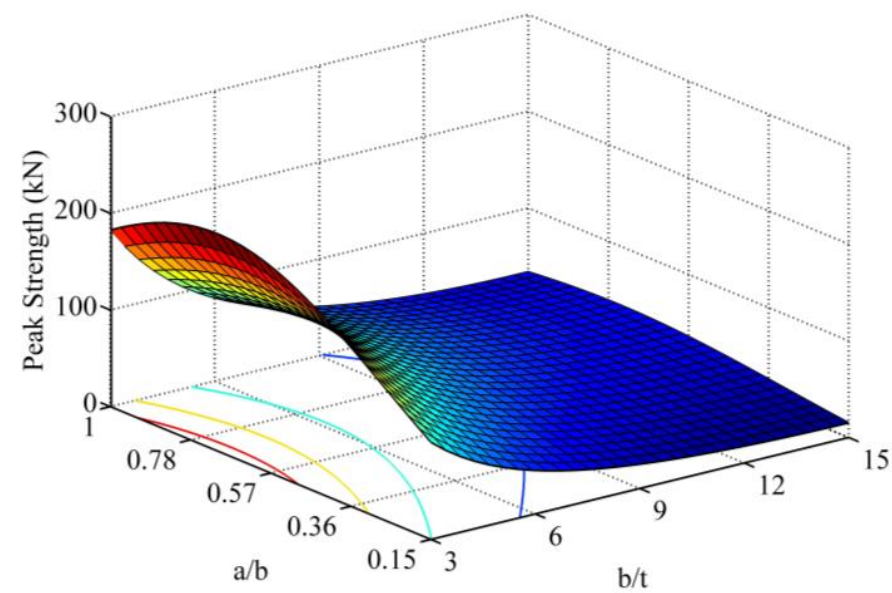

(c)

Figure 5. 5 Variation of maximum strength with respect to different pairs of input parameters.

\subsubsection{Ductility}

The ductility of steel plate fuses varies from 7.76 to 66.46 . The regression coefficients for the non-transformed response are presented in Tables 5.4 and 5.10. ANOVA shows adjusted $R^{2}$ value of 0.962 . The 3D response surface plots in Figure 5.7 illustrate the effects of two-factor interactions on the ductility response. 
Table $5.9 \beta_{i j}$ 's for ductility.

\begin{tabular}{ccccc}
\hline \multirow{3}{*}{$i$} & $\begin{array}{c}\text { Input parameter, } \\
x_{i} \text { and } x_{j}\end{array}$ & \multicolumn{3}{c}{$\beta_{i j}$ 's for $y$} \\
\cline { 3 - 5 } & & $L / b$ & $b / t$ & $a / b$ \\
\hline 1 & $L / b$ & -3.061 & 0.256 & 4.228 \\
2 & $b / t$ & & 0.539 & 1.013 \\
3 & $a / b$ & & -172.5 \\
\hline
\end{tabular}
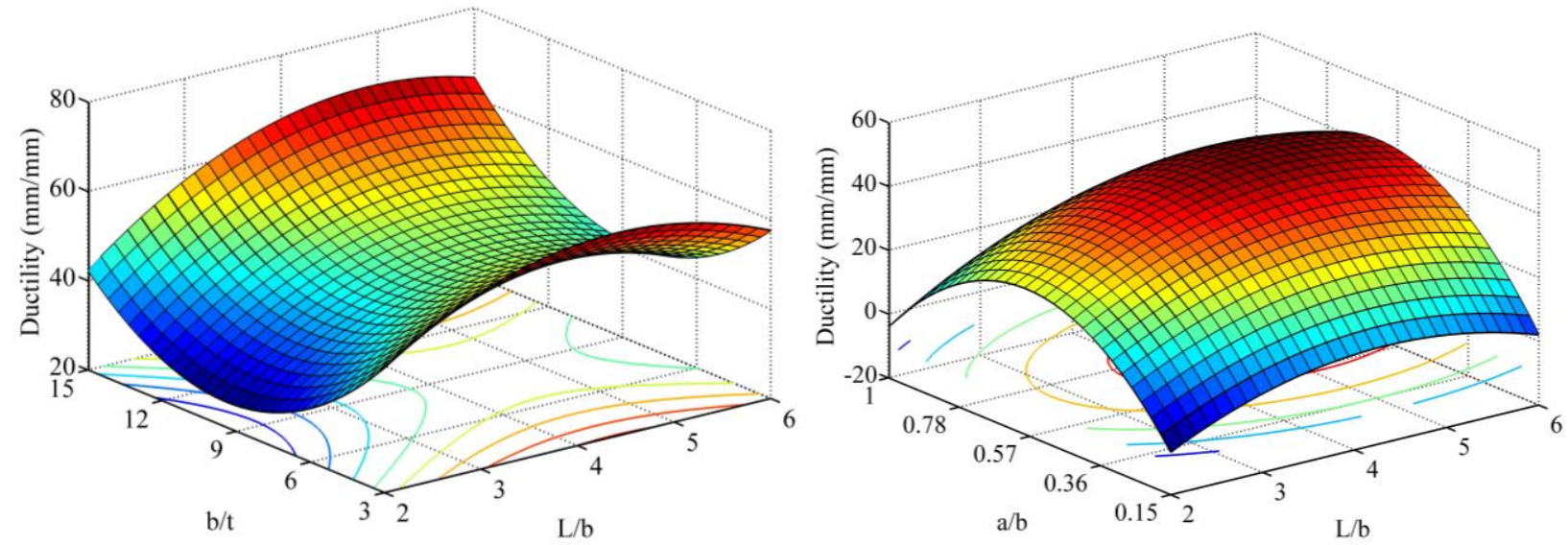

(a)

(b)

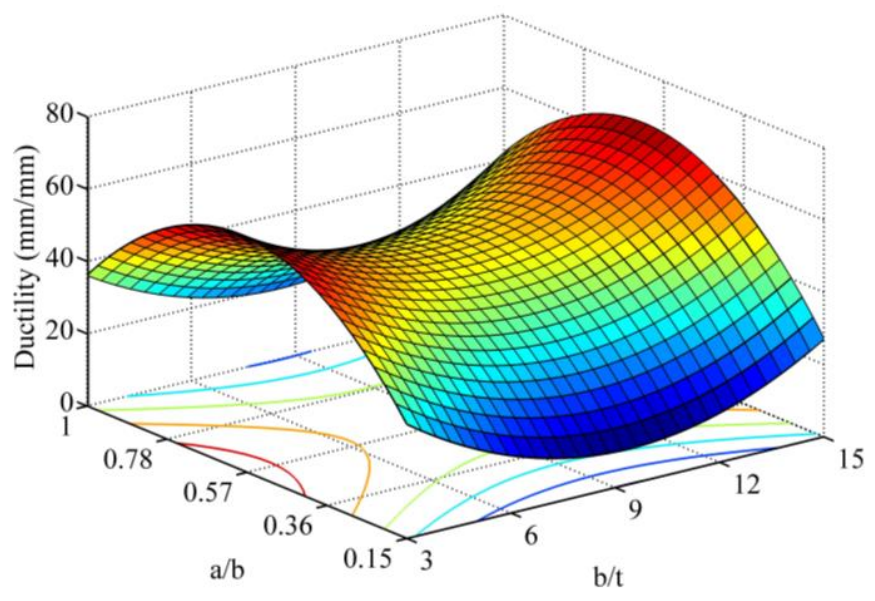

(c)

Figure 5. 6 Variation of ductility with respect to different pairs of input parameters. 


\subsection{Verification of the Response Surface Models}

The values of $R^{2}$-adjusted are greater than 0.95 for all the response characteristics. The predicted $R^{2}$ is greater than 0.80 for all predictive equations. Therefore, the response surface models can be used to accurately predict the response variables. In this section, the accuracy of the predictive equations is further assessed by performing additional $\mathrm{FE}$ simulations and comparing the response variables with those from the developed predictive equations.

Seven additional models of steel plate fuses are simulated in ANSYS. The input parameters for the additional models are chosen based on randomly generated combinations over the specified ranges in Table 5.1 (i.e. between the low and high levels). Table 5.11 provides the factor combinations that are used for the verification of the response surface models.

The accuracy of the predictive equations is assessed through the comparison of the actual response quantities with the predicted response using the response surface models. Table 5.12 provides the ratio of the response quantities using FE simulation to that using the predictive equations for each response characteristic. Figure 5.8 is used to compare the actual response values with the predicted response values obtained from the predictive equations. Adjusted $R^{2}$ values are 0.999, 0.992, 0.957, 0.993, and 0.962 for the initial stiffness, yield strength, effective damping, maximum strength, and ductility, respectively. Based on this verification study, the predictive equations can predict the response variables with an acceptable level of accuracy.

Table 5. 10 Factor combinations used for the verification of the response surface models.

\begin{tabular}{cccc}
\hline \multirow{3}{*}{ Model } & \multicolumn{3}{c}{ Factors } \\
\cline { 2 - 4 } & $L / b$ & $b / t$ & $a / b$ \\
\hline 1 & 5.32 & 3.75 & 0.67 \\
2 & 3.36 & 5.63 & 0.16 \\
3 & 5.24 & 5.29 & 0.43 \\
4 & 3.02 & 15.00 & 0.37 \\
5 & 2.09 & 4.29 & 0.47 \\
6 & 4.14 & 8.18 & 0.41 \\
7 & 2.78 & 3.33 & 0.26 \\
\hline
\end{tabular}


Table 5. 11 Response values for the verification of response surface models.

\begin{tabular}{cccccc}
\hline \multicolumn{5}{c}{ Ratio of the FE simulation response to the predicted response. } \\
\hline Model & $K$ & $P_{y}$ & $\beta_{\text {eff }}$ & $P$ & $\mu$ \\
\hline 1 & 0.96 & 0.96 & 0.81 & 0.93 & 0.85 \\
2 & 0.89 & 1.10 & 1.11 & 0.92 & 0.98 \\
3 & 0.98 & 0.92 & 0.86 & 1.07 & 1.03 \\
4 & 1.01 & 1.11 & 1.25 & 1.15 & 1.12 \\
5 & 0.96 & 1.00 & 0.89 & 0.89 & 0.86 \\
6 & 0.91 & 1.08 & 1.26 & 1.11 & 1.00 \\
7 & 1.00 & 1.10 & 1.05 & 0.90 & 1.00 \\
\hline
\end{tabular}




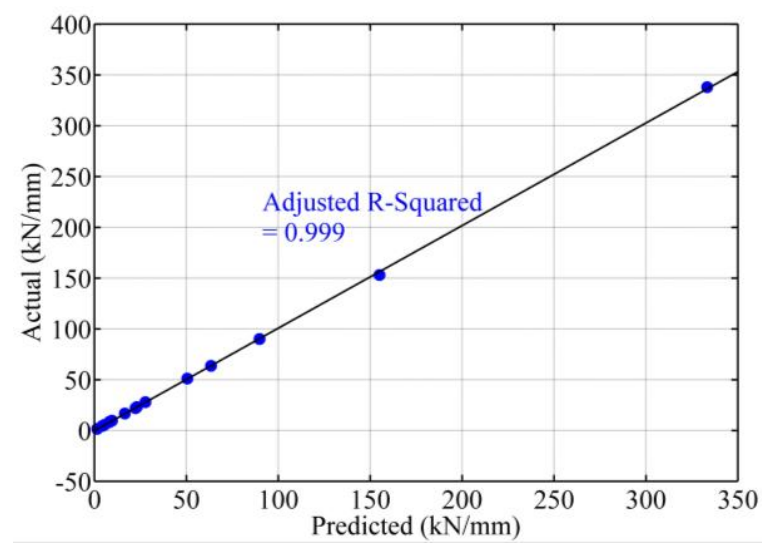

(a)

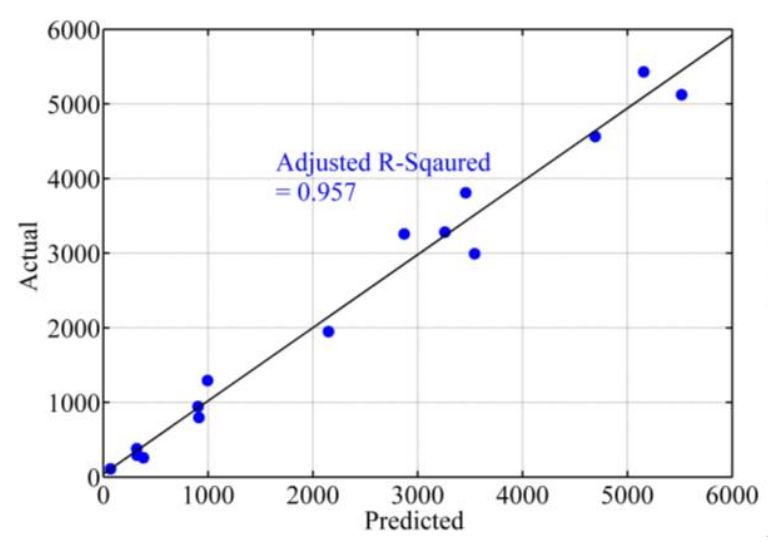

(c)

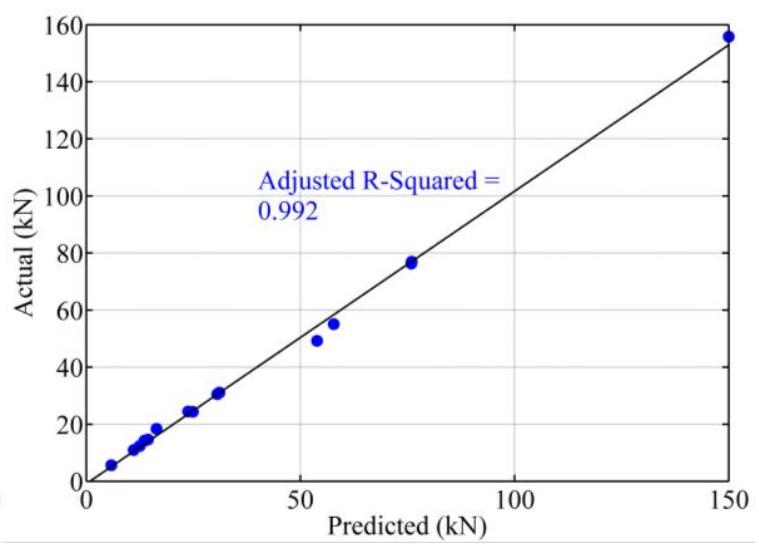

(b)

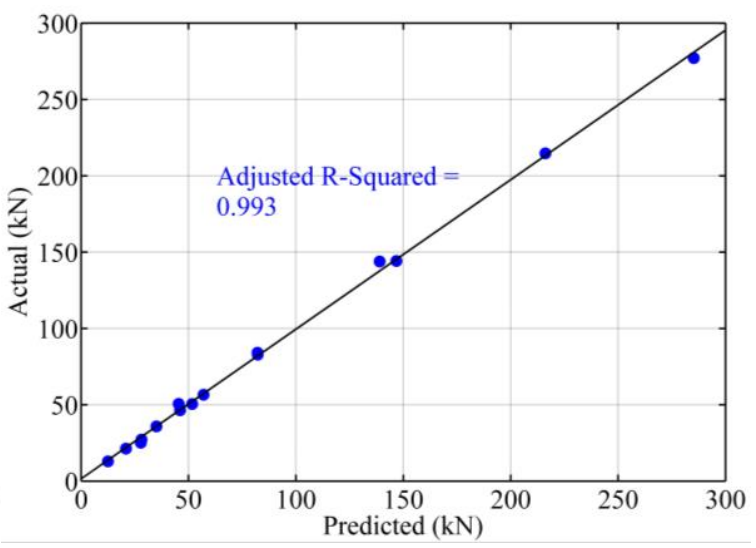

(d)

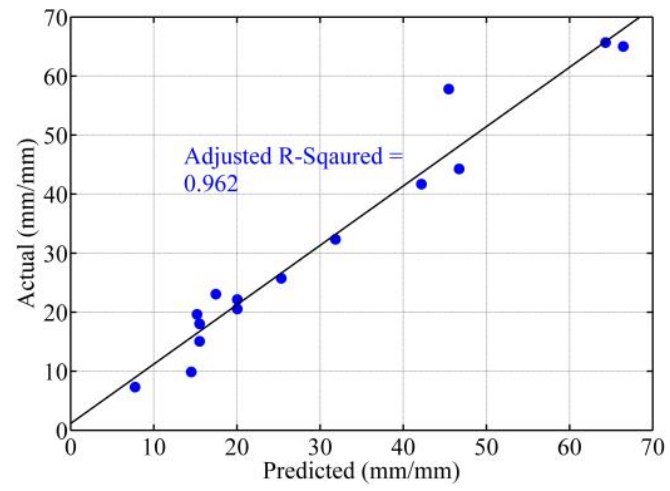

(e)

Figure 5. 7 Comparison of actual response with predicted response using response surface models. 


\subsection{Summary}

In chapter 5 the most significant factors that were obtained based the sensitivity study of Chapter 4 are considered for further study. The ratios of these factors are studied to derive predictive models for each response characteristics. These ratios are length to end-width, endwidth to thickness, and mid-width to end-width. Following the derivation of predictive equations additional simulation models, are run to confirm the accuracy of the predictive models. 


\section{CHAPTER 6 \\ MULTIPLE-RESPONSE OPTIMIZATION}

\subsection{Overview}

In this chapter the developed response surface models are used to optimize the response characteristics of energy dissipating steel plate fuses. Obtaining an optimal condition may require simultaneous consideration of multiple response values. Therefore, desirability functions are used for the optimization study.

\subsection{Desirability Functions for Multiple-response Optimization}

For this study, a multiple-response optimization is considered as compared to a univariate optimization. The simultaneous consideration of different response variables can be complex since the problem may involve conflicting objectives. Simultaneous optimization problems can be approached using a desirability function method introduced by Derringer and Suich [59]. Using desirability functions, first, $d_{i}$ is defined for each response value. This function demonstrates the desirability with respect to each response having the most and least desirable condition of 1 and 0 , respectively. Then, an overall desirability function, $D$ is defined and maximized [60]:

$$
D=\left(d_{1} \times d_{2} \times \ldots \times d_{n}\right)^{1 / n} \quad \text { Eq. (4) }
$$

In Eq. (4), $n$ is the number of responses in the optimization problem.

The desirability function for each response variable is defined differently depending on the objectives of the optimization problem. When the objective of the problem is to maximize the response variable, the desirability function is as follows:

$$
d=\left\{\begin{array}{cc}
0 & y<L \\
\left(\frac{y-L}{T-L}\right)^{w} & L \leq y \leq T \\
1 & y>T
\end{array}\right.
$$

Where $T$ and $L$ denote the target and lower bounds, respectively. The shape of the desirability function can be altered by putting different levels of emphasis on the response variables by employing weight, $w$. Weight varies from 0.1 to 10 [56]. A value of 10 indicates the most emphasis on the target. When the objective of the problem is to minimize the response variable, the desirability function will be in the form of Eq. (6): 


$$
d=\left\{\begin{array}{cc}
1 & y<T \\
\left(\frac{y-U}{T-U}\right)^{w} & T \leq y \leq U \\
0 & y>U
\end{array}\right.
$$

\subsection{Optimization Goals}

For the optimization of steel plate fuses, several objectives are considered in four different optimization problems as shown in Table 6.1. These goals involve improving the structural response characteristics of steel plate fuses in terms of maximizing the effective damping and ductility; minimizing the yield strength and maximizing the effective damping and ductility; maximizing the initial stiffness, effective damping and ductility; and maximizing the peak strength, effective damping and ductility.

Maximizing the effective damping and ductility is common in all the optimization problems as highly ductile behavior and maximized energy dissipation capacity are critical for steel plate fuses. For a well-designed steel plate fuse, the lateral displacements under lateral loads should be reduced, in which this is achieved by increasing the initial stiffness [13]. Yield strength of steel plate fuses should be low enough in order to concentrate the damage on the plate at an early step of loading and limit the damage to other main structural components. Steel plate fuses also may be expected to possess a greater load capacity. In all the optimization problems, weight, $w$, is set to 1 .

Table 6. 1 Optimization objectives.

\begin{tabular}{lccccc}
\hline & \multicolumn{5}{c}{ Response } \\
\cline { 2 - 5 } & $K$ & $P_{y}$ & $\beta_{\text {eff }}$ & $P$ & $\mu$ \\
\hline In optimization 1 & & Maximize & Maximize \\
In optimization 2 & & Minimize & Maximize & & Maximize \\
In optimization 3 & Maximize & & Maximize & & Maximize \\
In optimization 4 & & & Maximize & Maximize & Maximize \\
\hline
\end{tabular}




\subsection{Results and Discussions of the Optimization Studies}

\subsubsection{Results of Optimization Problem 1}

The first optimization problem determines the optimum regions of the design space by maximizing the effective damping and ductility. One optimal parameter setting is found to be: length to end-width ratio $=4.55$, end-width to thickness ratio $=3.00$, and mid-width to end-width ratio $=0.598$. The overall desirability function, $D$, for this parameter combination is 0.992 , which is determined using Equations (6) and (7) as follows:

$(d)_{\mu}=\left(\frac{y-L}{T-L}\right)^{w}=\left(\frac{65.480-7.760}{66.462-7.760}\right)^{1}=0.983 \quad$ Eq. (7)

$D=(0.983 \times 1)^{1 / 2}=0.992 \quad$ Eq. $(8)$

Figure 6.1 shows one-factor desirability plots at the optimum condition. These plots indicate the change in the desirability function over the range of each individual parameter. To achieve the most desirable condition of the response characteristic corresponding to an optimal range for each factor, a desirability value of $79 \%$ or greater is considered. This limit is in fact $80 \%$ of the observed maximum desirability $(80 \% \times 0.992=79 \%)$. 


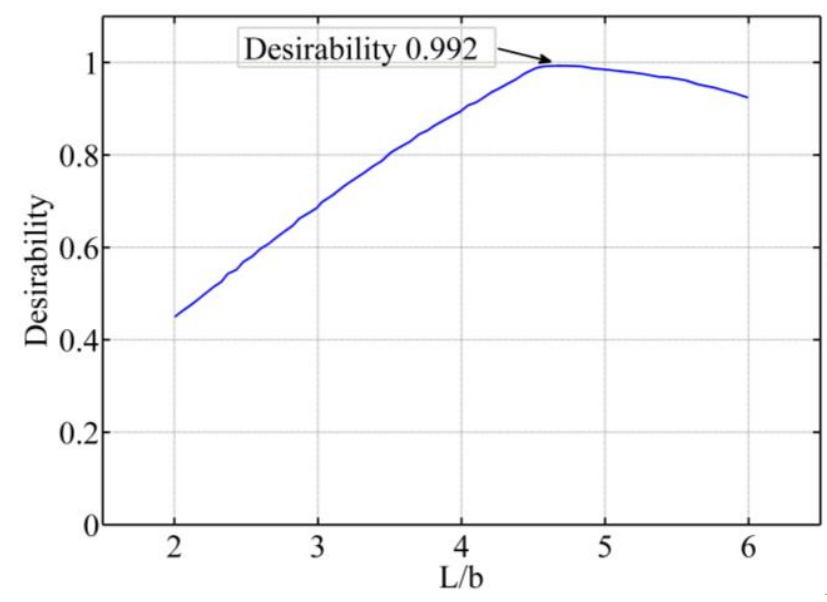

(a)

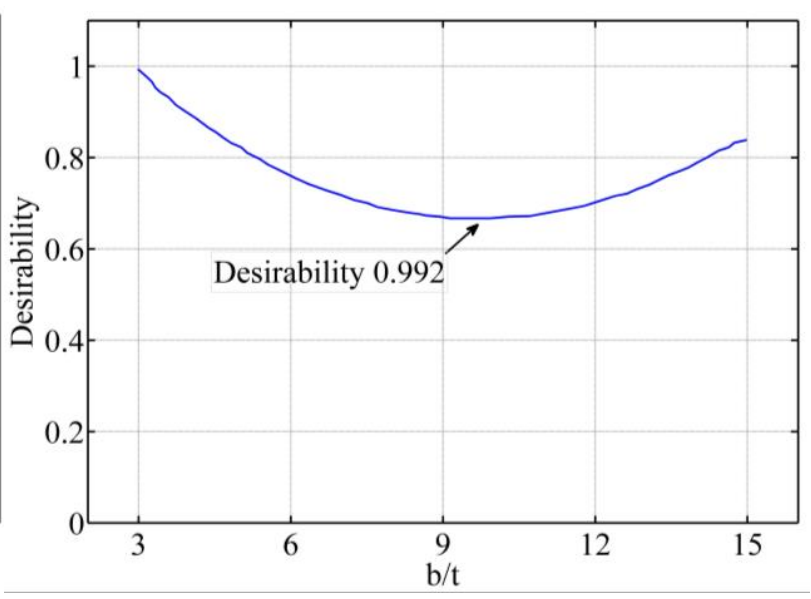

(b)

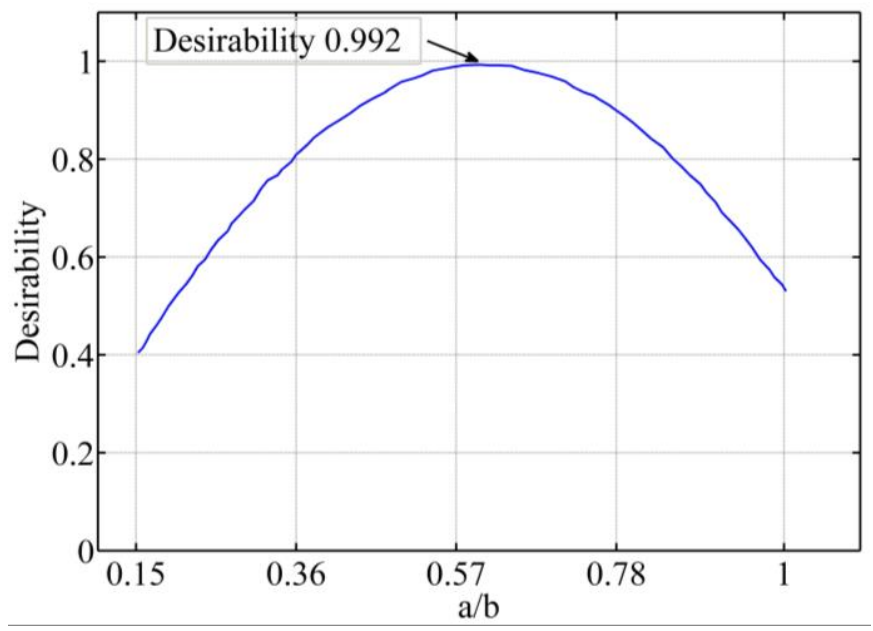

(c)

Figure 6. 1 The variation of desirability function versus input parameters for optimization problem 1.

As observed in Figure 6.1(a) a desirability value equal to or higher than $79 \%$ is achieved when the $L / b$ value of the steel plate fuse is between 3.5 and 6 . This indicates that moderate to higher ranges of $L / b$ are desirable for maximizing the effective damping and ductility response characteristics. 
Figure 6.1(b) indicates that the desirability function is varying over different limits of $b / t$ in steel plate fuses. It is observed that the desirability remains greater than $79 \%$ when the $b / t$ values are either smaller than 5.5 or greater than 14 .

In Figure 6.1(c), it is observed that mid-range values of $a / b$ are significantly desirable in maximizing the effective damping and ductility response values. The optimal range for the $a / b$ value of steel plate fuses is in between 0.35 and 0.86 . This optimal range demonstrates a desirability value of greater than or equal to $79 \%$.

Considering the variability in the optimal ranges of each factor indicated in Figure 6.1, the most effective factors on the overall desirability function can be evaluated. Table 6.2 indicates the ranking of the most influential factors to least in maximizing the effective damping and ductility response variables for steel plate fuses. The ranking of effective factors is defined based on the variability in the desirability function over the range of the specified factor. For the first optimization problem, the most effective factor is found to be the mid-width to end-width ratio while end-width to thickness ratio is the least influential factor. In Table 6.2, the optimal ranges of each individual factor having a desirability equal to or greater than $79 \%$, is also summarized. Further, for the goal of improving the effective damping and ductility, optimal ranges are described for each factor in the last column of Table 6.2.

Table 6. 2 Ranking of the most influential input parameters to least for optimization problem 1.

\begin{tabular}{ccccccc}
\hline Rank & Input Parameter & $\Delta \mathrm{D}$ & \multicolumn{2}{c}{ Optimal range } & Unit & Higher Desirability \\
\cline { 3 - 5 } & & & Low & High & & \\
\hline 1 & $a / b$ & 0.59 & 0.35 & 0.86 & - & Moderate \\
2 & $L / b$ & 0.54 & 3.5 & 6 & - & Moderate/high \\
3 & $b / t$ & 0.33 & $\leq 5.5$ & $\geq 14$ & - & Low/high \\
\hline
\end{tabular}

For further support of the above results, contour plots shown in Figure 6.2 are used to present the desirability function for different pairs of factors. The optimal condition with the desirability of $79 \%$ is demonstrated in the plots. 


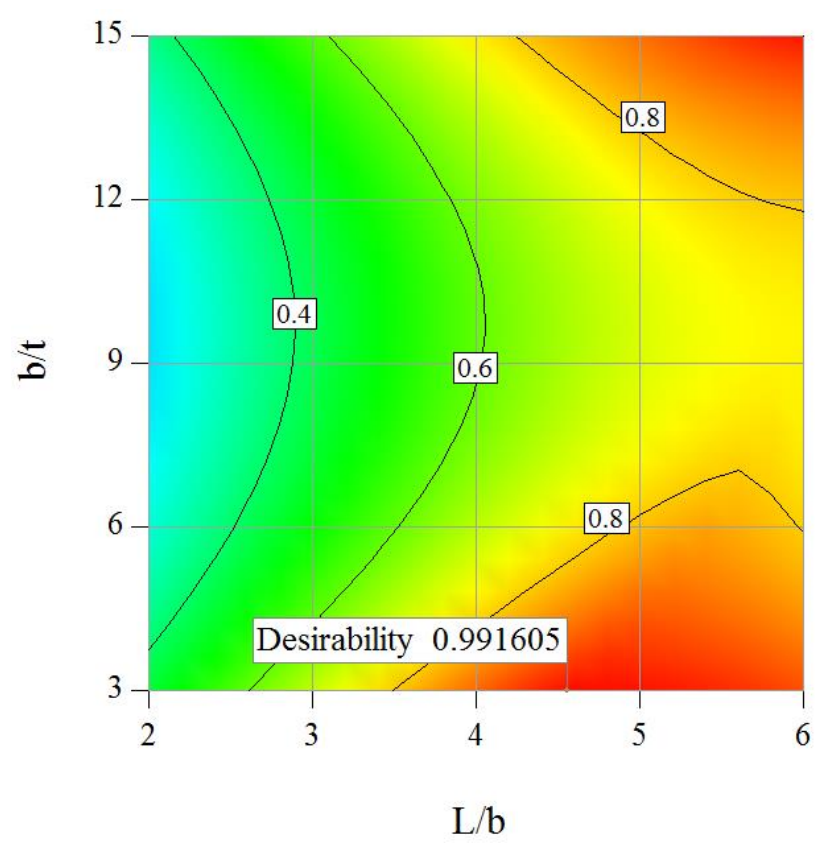

(a)

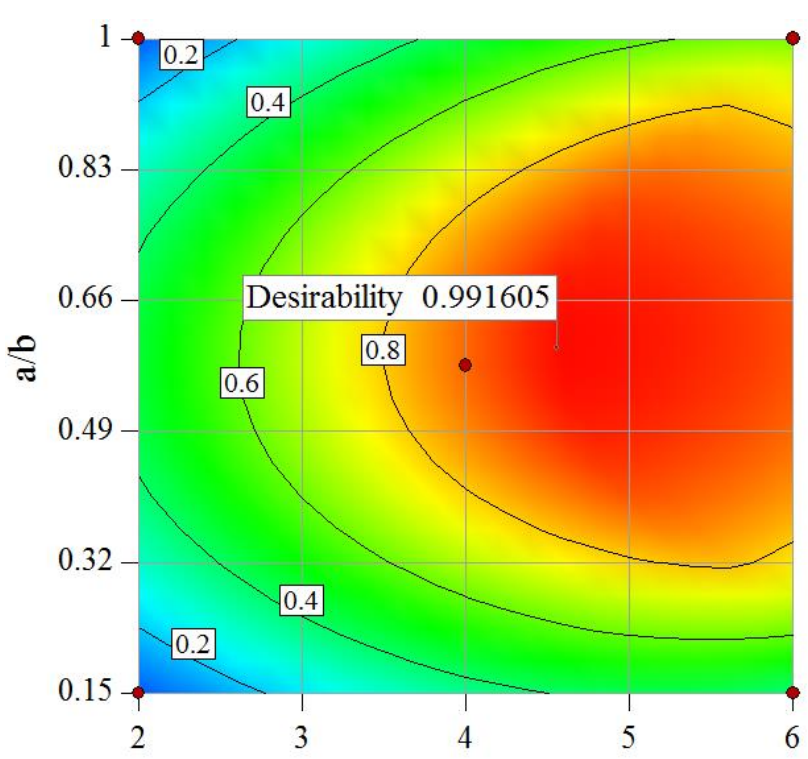

L/b

(b)

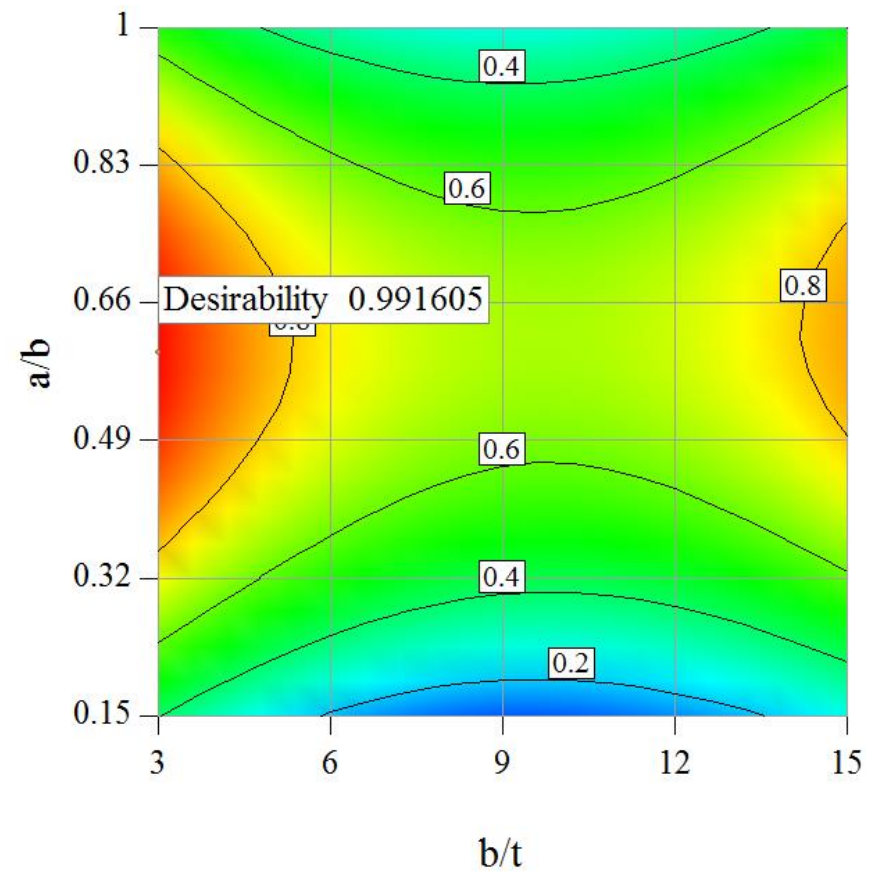

(c)

Figure 6. 2 Contour plots of desirability functions for optimization problem 1.

\subsubsection{Results of Optimization Problem 2}

The second optimization problem is used to identify the optimum ranges for the design space in terms of minimizing and maximizing the structural response variables, which include the 
minimization of yield strength; and maximization of the effective damping and ductility. Among the evaluated optimized solutions, one optimal parameter setting that leads to achieving the specified goal is found to be: length to end-width ratio $=6.00$, end-width to thickness ratio $=15.00$, and mid-width to end-width ratio $=0.64$. The overall desirability function $(D)$ for this factor combination is 0.977 . The overall desirability function is calculated from the desirability functions of each individual response value in the optimization, as shown in Eq. (9).

$D=(0.961 \times 0.989 \times 0.980)^{1 / 3}=0.977 \quad$ Eq. (9)

Figure 6.3 illustrates the variation of the desirability function with respect to each design parameter. The minimization of yield strength; and maximization of effective damping and ductility is reached through the optimal condition shown in these one-factor desirability plots. The maximum desirability value for the defined objective is $D=0.977$. Considering the optimal ranges of each individual design parameter $80 \%$ of the peak desirability $(80 \% \times 0.977=$ 0.782 ) is considered. This limit is used for the specification of an optimal range, where a desirability value of equal to or higher than 0.782 is reached. 


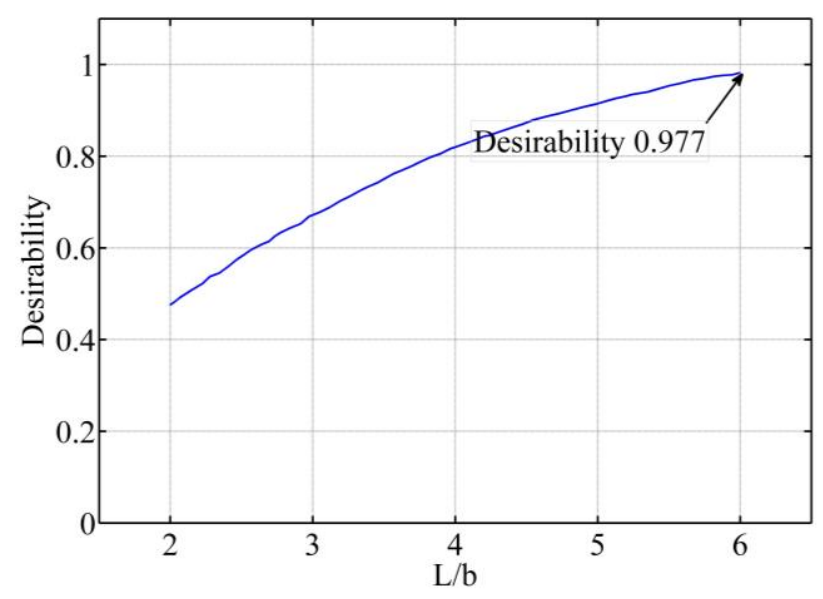

(a)

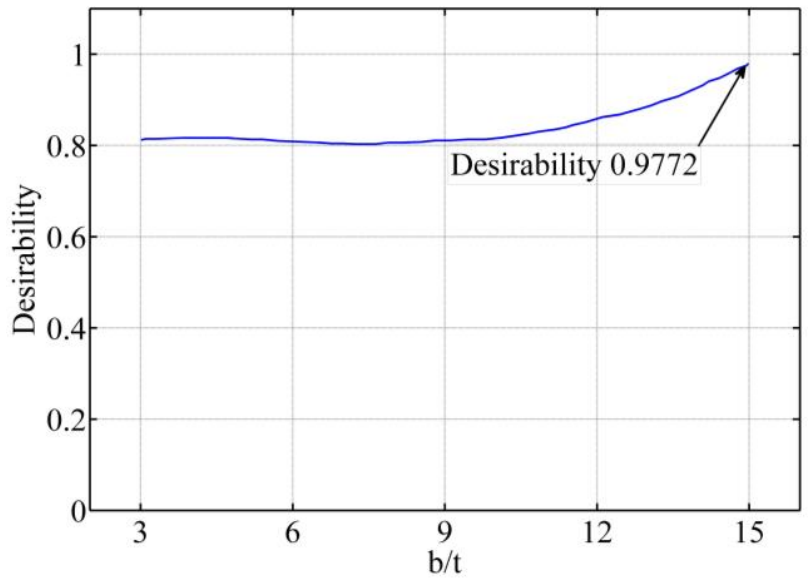

(b)

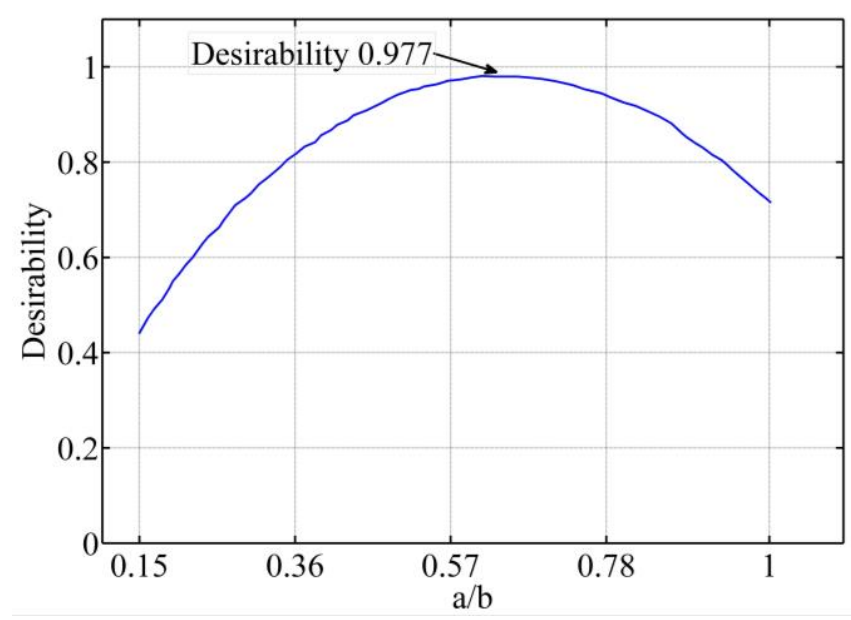

(c)

Figure 6. 3 The variation of desirability function versus input parameters for optimization problem 2.

As observed in Figure 6.3(a), the desirability value equal to or higher than $78.2 \%$ is achieved when the length to end-width ratio of the steel plate fuse is between 3.73 and 6 . This demonstrates that, desirable effective damping and ductility is reached when moderate to higher ratios of length to end-width is considered for steel plate fuses.

Figure 6.3(b) suggests that the variation of end-width to thickness factor over the given range in steel plate fuses is not effective in minimizing the yield strength along with the maximization of the effective damping and ductility responses. The overall range of end-width to thickness shows a desirability of greater than or equal to 0.782 for optimizing steel plate fuses 
(increased response characteristic in terms of effective damping and ductility; and decreased response characteristic in terms of yield strength).

In Figure 6.3(c) it is seen that moderate ratios of mid-width to end-width lead to higher desirability functions in achieving minimized yield strength; and maximized effective damping and ductility. The optimal range for the mid-width to end-width ratio of steel plate fuses is from 0.33 to 0.95 . This optimal range shows a desirability value of greater or equal to $78.2 \%$.

Based on the variability of optimal ranges for each factor indicated in Figure 6.3, the most effective factors on the overall desirability function is summarized in Table 6.3. The evaluation of the most influential factors to least in minimizing the yield strength and maximizing the effective damping and ductility response variables for steel plate fuses is described in the ranking column. For this optimization problem the most effective factor is found to be the mid-width to end-width ratio while end-width to thickness ratio is the least influential factor. In Table 6.3 the optimal ranges of each individual factor having a desirability equal or greater than $78.2 \%$, is also discussed. The optimal ranges can also be investigated using the contour plots of desirability function for different pairs of factors. These plots are illustrated in Figure 6.4. The results described above are further supporter by the contour plots.

Table 6. 3 Ranking of the most influential input parameters to least for optimization problem 2.

\begin{tabular}{ccccccc}
\hline Rank & Input Parameter & $\Delta \mathrm{D}$ & \multicolumn{2}{c}{ Optimal range } & Unit & Higher Desirability \\
\cline { 3 - 5 } & & & Low & High & & \\
\hline 1 & $a / b$ & 0.54 & 0.33 & 0.95 & - & Moderate \\
2 & $L / b$ & 0.50 & 3.73 & 6.00 & & Moderate/high \\
3 & $b / t$ & 0.18 & 3.00 & 15.0 & - & Low/moderate/high \\
\hline
\end{tabular}




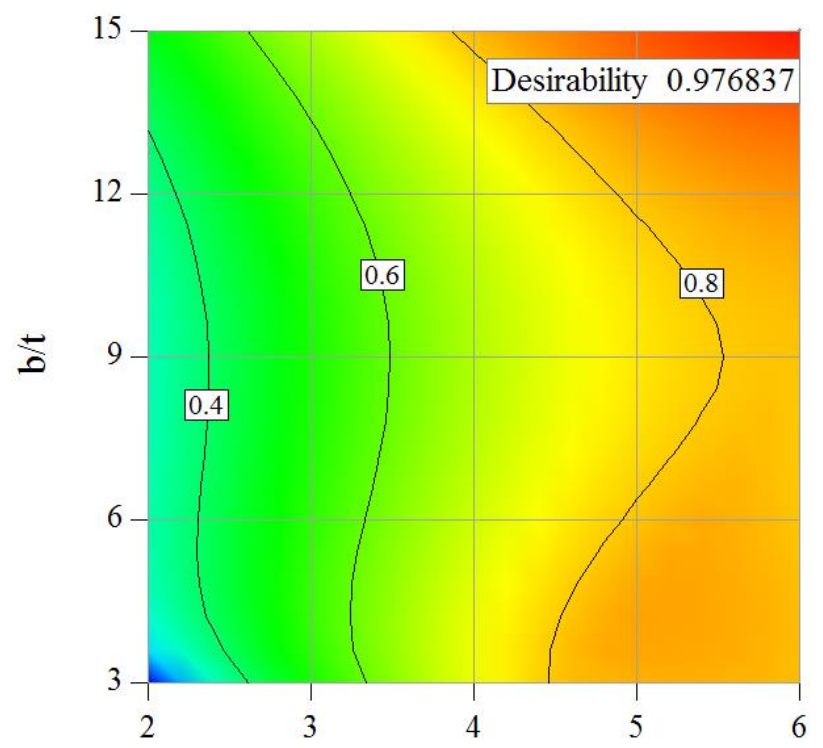

$\mathrm{L} / \mathrm{b}$

(a)

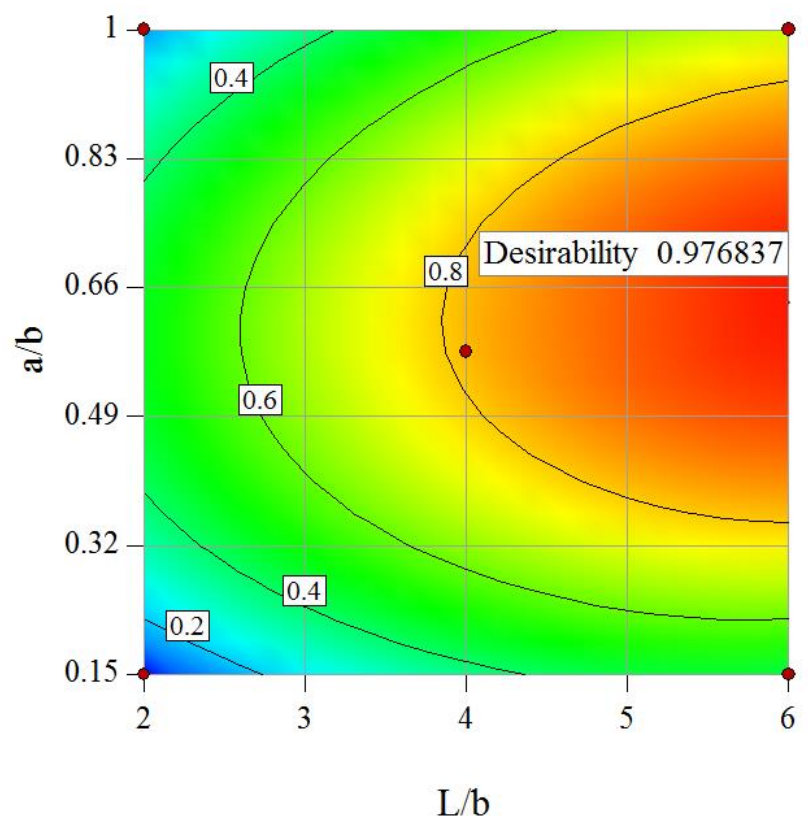

(b)

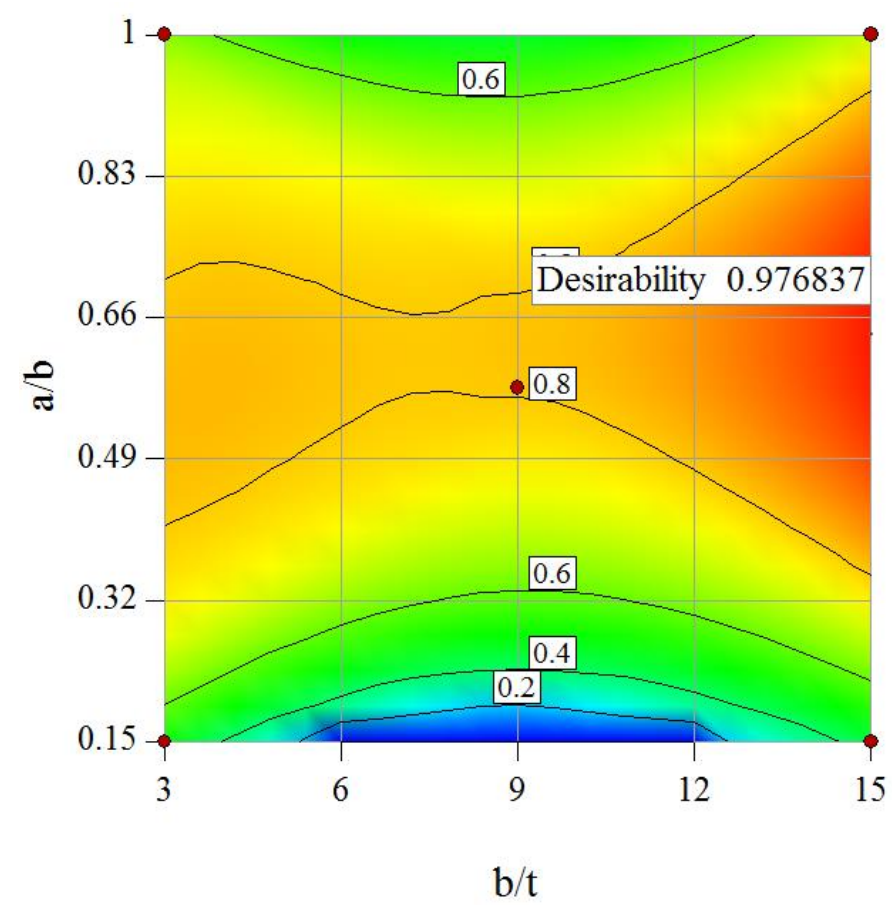

(c)

Figure 6. 4 Contour plots of desirability functions for optimization problem 2. 


\subsubsection{Results of Optimization Problem 3}

This problem is used to identify the optimum ranges of each design parameter that leads to achieving the goal of maximizing the initial stiffness, effective damping and ductility response characteristics. One optimal parameter combination is found to be: length to end-width ratio = 2.30 , end-width to thickness ratio $=3.00$, and mid-width to end-width ratio $=0.625$. This optimal parameter setting has an overall desirability function of 0.559. Eq. (10) is used to calculate the overall desirability function from the desirability functions of each individual response value in the optimization.

$D=(0.646 \times 0.363 \times 0.745)^{1 / 3}=0.559 \quad$ Eq. $(10)$

Figure 6.5 demonstrates the optimal conditions of one-factor desirability function. These plots show the variation in the desirability function over the range of each individual parameter. The goal of the problem is reached based on the consideration of $80 \%$ of the peak desirability $(80 \% \times 0.559=0.447)$. To achieve the most desirable response characteristic for an optimal range of each factor, a desirability value of $44.7 \%$ or greater $(D=0.447)$ is considered. 


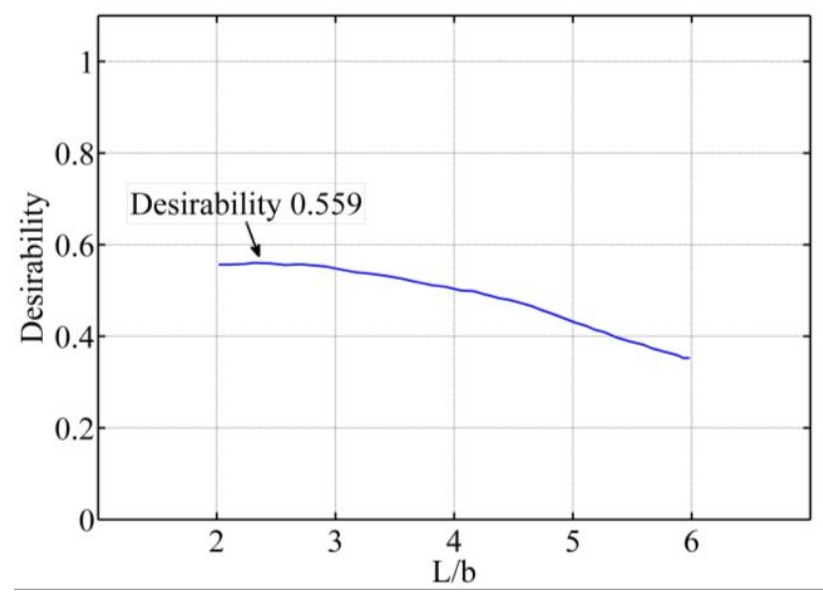

(a)

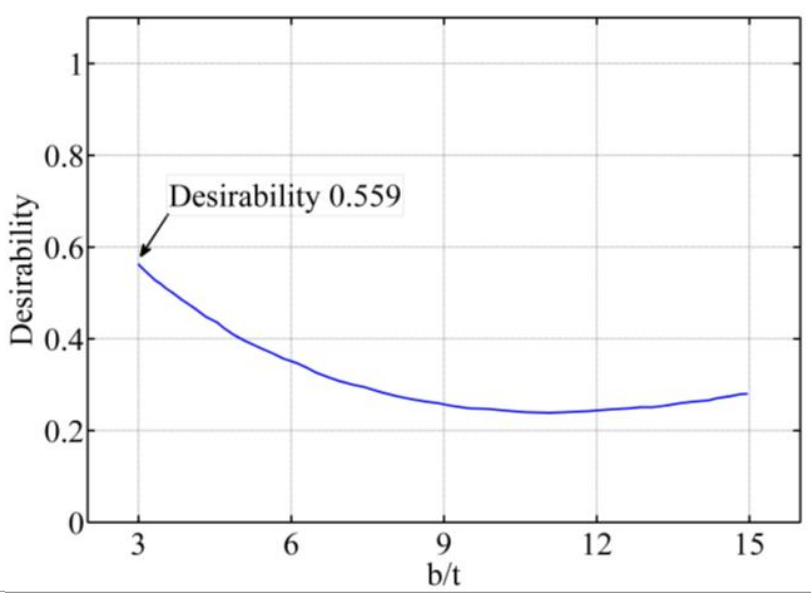

(b)

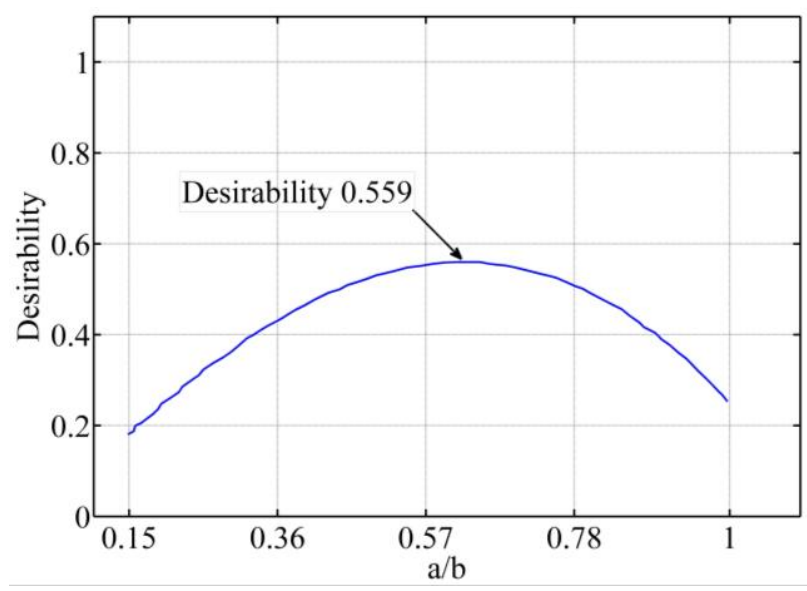

(c)

Figure 6. 5 The variation of desirability function versus input parameters for optimization problem 3.

While considering the length to end-width ratio of steel plate fuses, lower to moderate ratios are found to be more desirable (shown in Figure 6.5(a)). The desirability function remains greater than or equal to 0.447 when the length to end-width ratio is varied from 2 to 4.45 . Therefore, greater desirability value is achieved for a low to moderate range of length to end-width ratio. 
In Figure 6.5(b) it is observed that lower values of end-width to thickness ratio lead to higher desirability functions. The optimization goals of this problem can be reached with the optimal range of end-width to thickness ratios varying from 3.00 to 4.03 . This optimal range shows a desirability value of greater or equal to $44.7 \%$.

As for the steel plate fuse mid-width to end-width factor, the desirability plot is significantly affected with the variation in the mid-width to end-width ratio (shown in Figure $6.5(c))$. The desirability value remains greater than or equal to 0.447 when the mid-width to endwidth factor is changed between 0.38 to 0.82 over its entire range (from lower level to higher level). Therefore, it is seen that the moderate ranges of mid-width to end-width factor is desirable on reaching the optimization goals.

Table 6.4 summarizes the findings described above. This table also shows the ranking of each factor in terms of the overall change in the desirability function. For instance, as provided in Table 6.4, the mid-width to end-width factor is the most effective factor affecting the optimization goals while the end-width to thickness is seen to be the least influential factor. The above findings are further supported with the contour plots of desirability pairs of factors shown in Figure 6.6.

Table 6. 4 Ranking of the most influential input parameters to least for optimization problem 3.

\begin{tabular}{ccccccc}
\hline Rank & Input Parameter & $\Delta \mathrm{D}$ & \multicolumn{2}{c}{ Optimal range } & Unit & Higher Desirability \\
\cline { 3 - 5 } & & & Low & High & & \\
\hline 1 & $a / b$ & 0.38 & 0.38 & 0.82 & - & Moderate \\
2 & $b / t$ & 0.32 & 3.00 & 4.03 & - & Low \\
3 & $L / b$ & 0.21 & 2.00 & 4.45 & - & Low/moderate \\
\hline
\end{tabular}




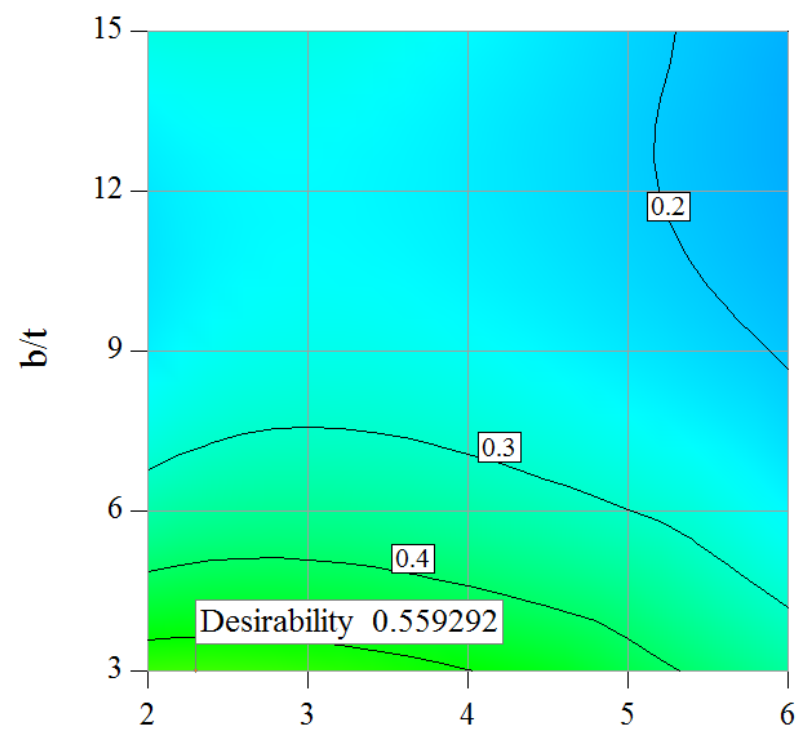

$\mathrm{L} / \mathrm{b}$

(a)

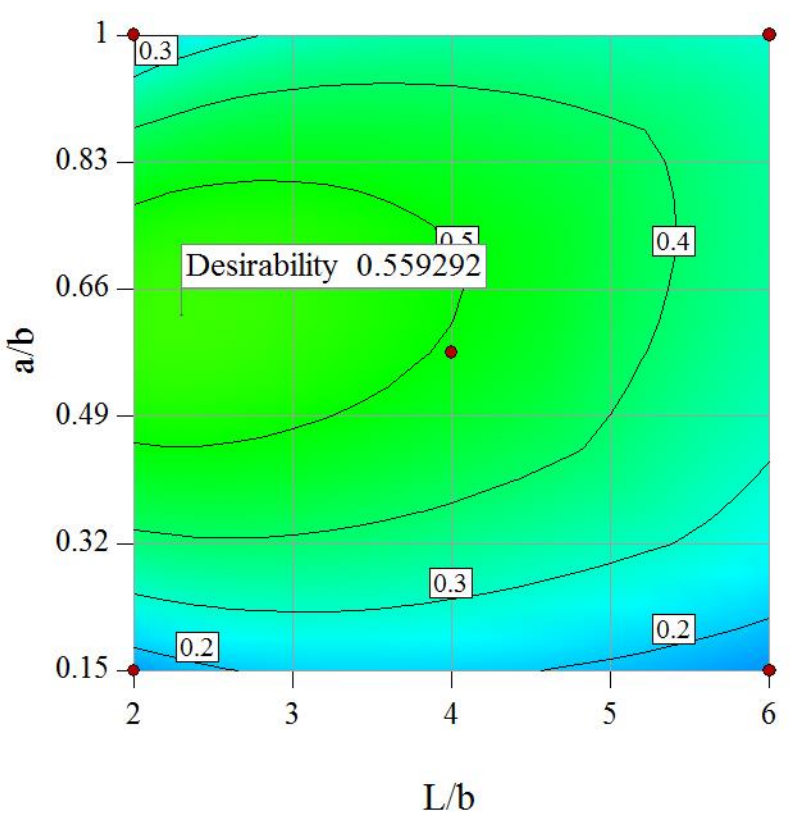

(b)

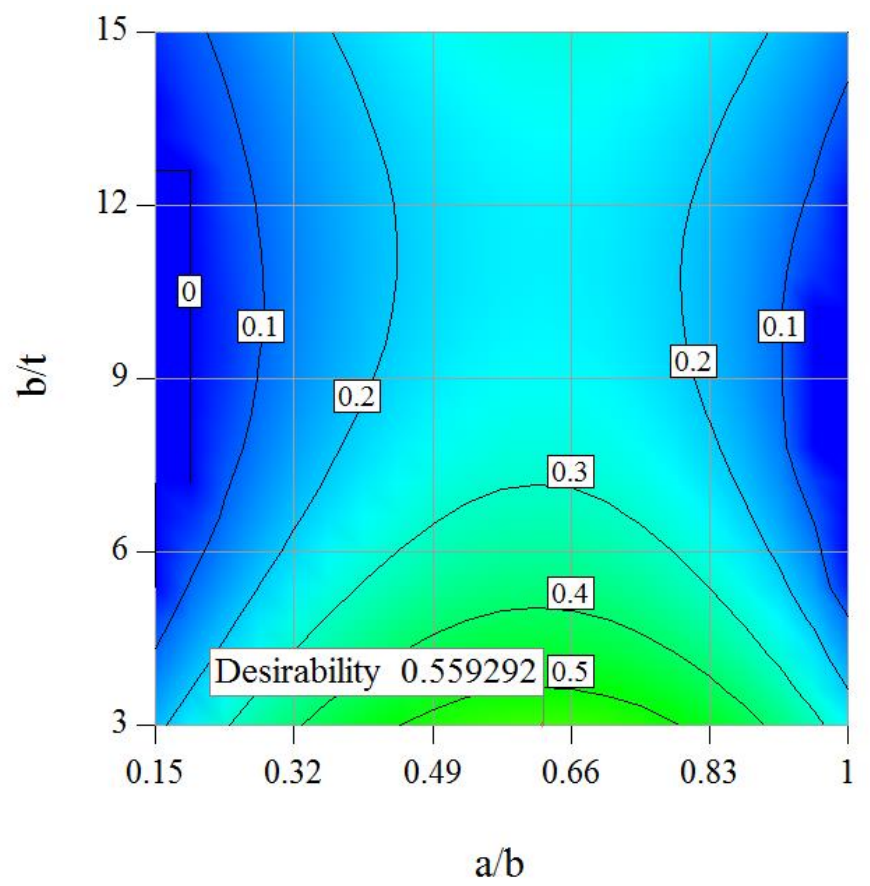

(c)

Figure 6. 6 Contour plots of desirability functions for optimization problem 3.

\subsubsection{Results of Optimization Problem 4}

The fourth optimization problem is used to determine the optimum ranges for the design space in terms of maximizing the structural response variables including the maximization of 
maximum strength, effective damping and ductility. Considering the evaluated optimized solutions, one optimal factor setting that leads to achieving the specified optimization goals is found to be: length to end-width ratio $=4.56$, mid-width to end-width ratio $=3.00 \mathrm{~mm}$, and midwidth to end-width ratio $=0.641$. The overall desirability function $(D)$ for this parameter setting is 0.885. The overall desirability function is calculated from the desirability functions of each individual response value in the optimization, as shown in Eq. (11).

$D=(1 \times 0.708 \times 0.977)^{1 / 3}=0.885$

Eq. (11)

Figure 6.7 illustrates the change in the desirability function with respect to each design parameter. The maximization of maximum strength, effective damping and ductility is achieved through the optimal condition shown in these one-factor desirability plots. The maximum desirability value for the optimization objective is 0.885 . Considering the optimal ranges of each individual design parameter, $80 \%$ of the peak desirability $(80 \% \times 0.885=0.708)$ is considered. This limit is used for the specification of an optimal range, where a desirability value of equal to or higher than 0.708 is achieved.

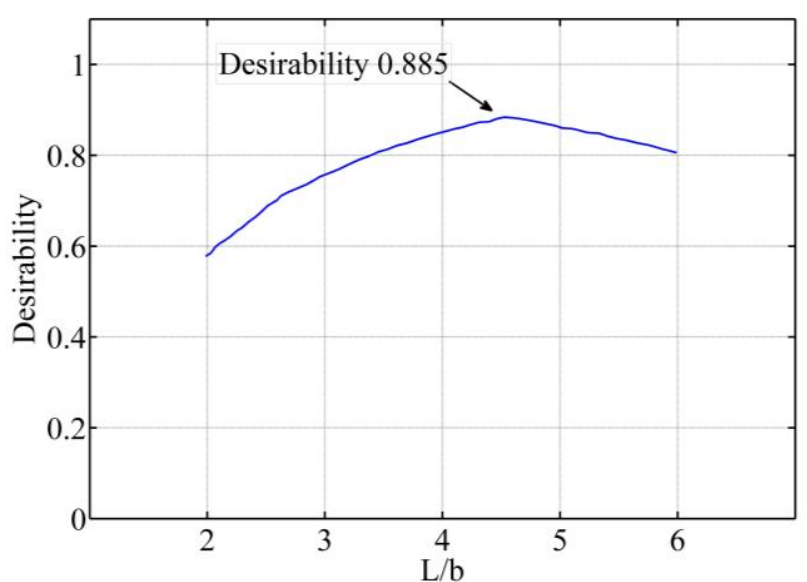

(a)

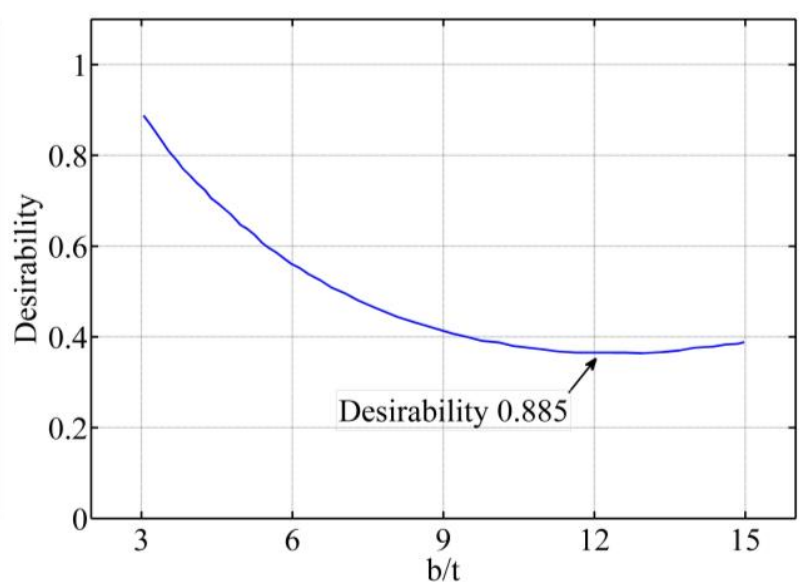

(b) 


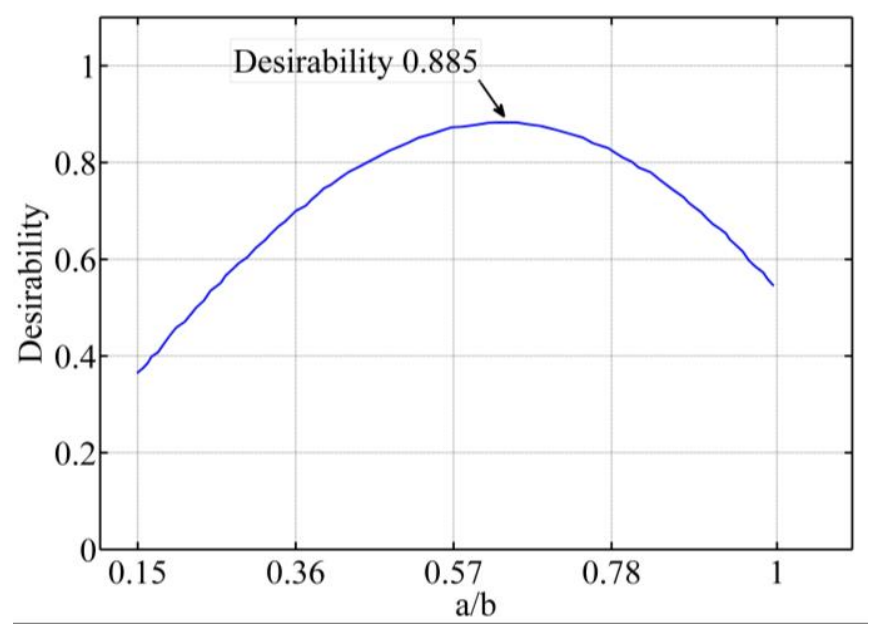

(c)

Figure 6. 7 The variation of desirability function versus input parameters for optimization problem 4.

As observed in Figure 6.7(a), the desirability value equal to or higher than $70.8 \%$ is reached when the length to end-width ratio of the steel plate fuse is varied from 2.64 and 6 . This indicates that, desirable maximum strength, effective damping and ductility is reached when low, moderate to higher values of length to end-width is considered for steel plate fuses.

Figure 6.7(b) presents that the change in end-width to thickness factor over the given range in steel plate fuses has a noticeable impact in achieving the optimization goals. Lower ranges of end-width to thickness ratio shows a desirability of greater than or equal to 0.708 for optimizing steel plate fuses (increased response characteristic in terms of maximum strength, effective damping and ductility).

In Figure 6.7(c) it is observed that moderate values of mid-width to end-width factor lead to higher desirability functions in achieving the optimization goals. The optimal range for the midwidth to end-width ratios of steel plate fuses is from 0.37 and 0.89 . This optimal range shows a desirability value of greater or equal to $70.8 \%$.

Looking at the variability of optimal ranges for each factor presented in Figure 6.7, the most effective factors on the overall desirability function is described in Table 6.5. The evaluation of the most influential factors to least in reaching the optimization goals for steel plate fuses is described in the ranking column. For this optimization problem the most effective factor is found 
to be the end-width to thickness ratio while length to end-width factor is the least influential factor. In Table 6.5 the optimal ranges of each individual factor having a desirability equal or greater than $70.8 \%$, is also provided. The optimal ranges can also be investigated using the contour plots of desirability function for different pairs of factors. These plots are provided in Figure 6.8. The results described above are further supporter by the contour plots.

Table 6. 5 Ranking of the most influential input parameters to least for optimization problem 4.

\begin{tabular}{ccccccc}
\hline Rank & Input Parameter & $\Delta \mathrm{D}$ & \multicolumn{2}{c}{ Optimal range } & Unit & Higher Desirability \\
\cline { 3 - 5 } & & & Low & High & & \\
\hline 1 & $b / t$ & 0.52 & 3.00 & 4.35 & - & Low \\
2 & $a / b$ & 0.52 & 0.37 & 0.89 & - & Moderate \\
3 & $L / b$ & 0.31 & 2.64 & 6 & - & Low/moderate/high \\
\hline
\end{tabular}




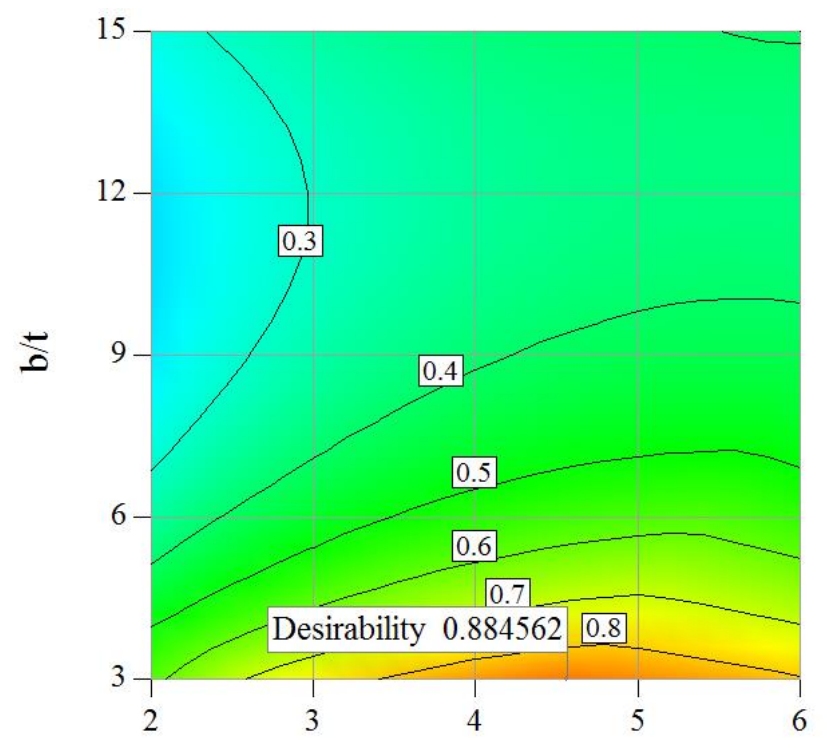

$\mathrm{L} / \mathrm{b}$

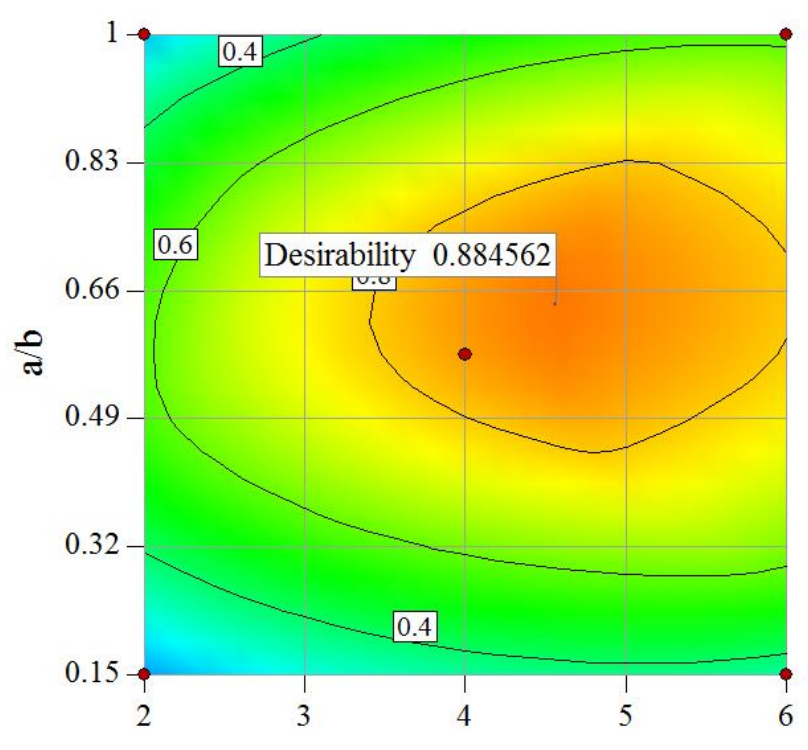

$\mathrm{L} / \mathrm{b}$

(a)

(b)

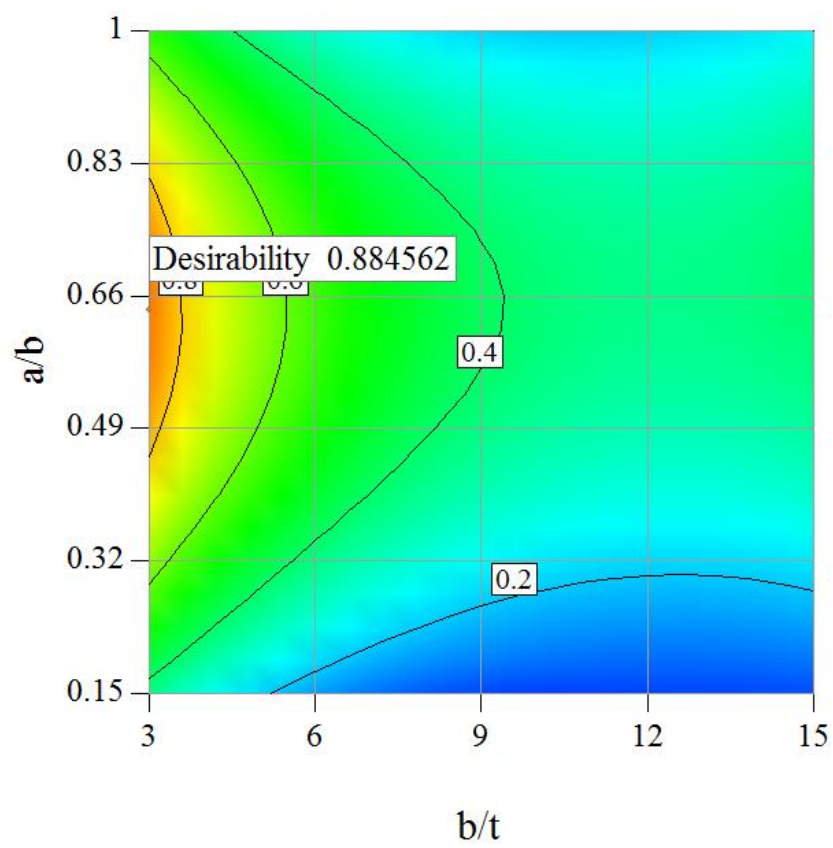

(c)

Figure 6. 8 Contour plots of desirability functions for optimization problem 4.

\subsection{Confirmation of the Optimization Results}

In order to assess the accuracy of the optimization results, four confirmation runs are performed in ANSYS with the optimal factor combinations obtained from the optimization studies. 
Table 6.6 summarizes a comparison of the response characteristics from the FE analysis in ANSYS and the optimal solutions. The confirmation study indicates that the predicted optimal response agrees with the simulated result, thus the optimization results are valid.

Table 6. 6 Confirmation of the optimization results.

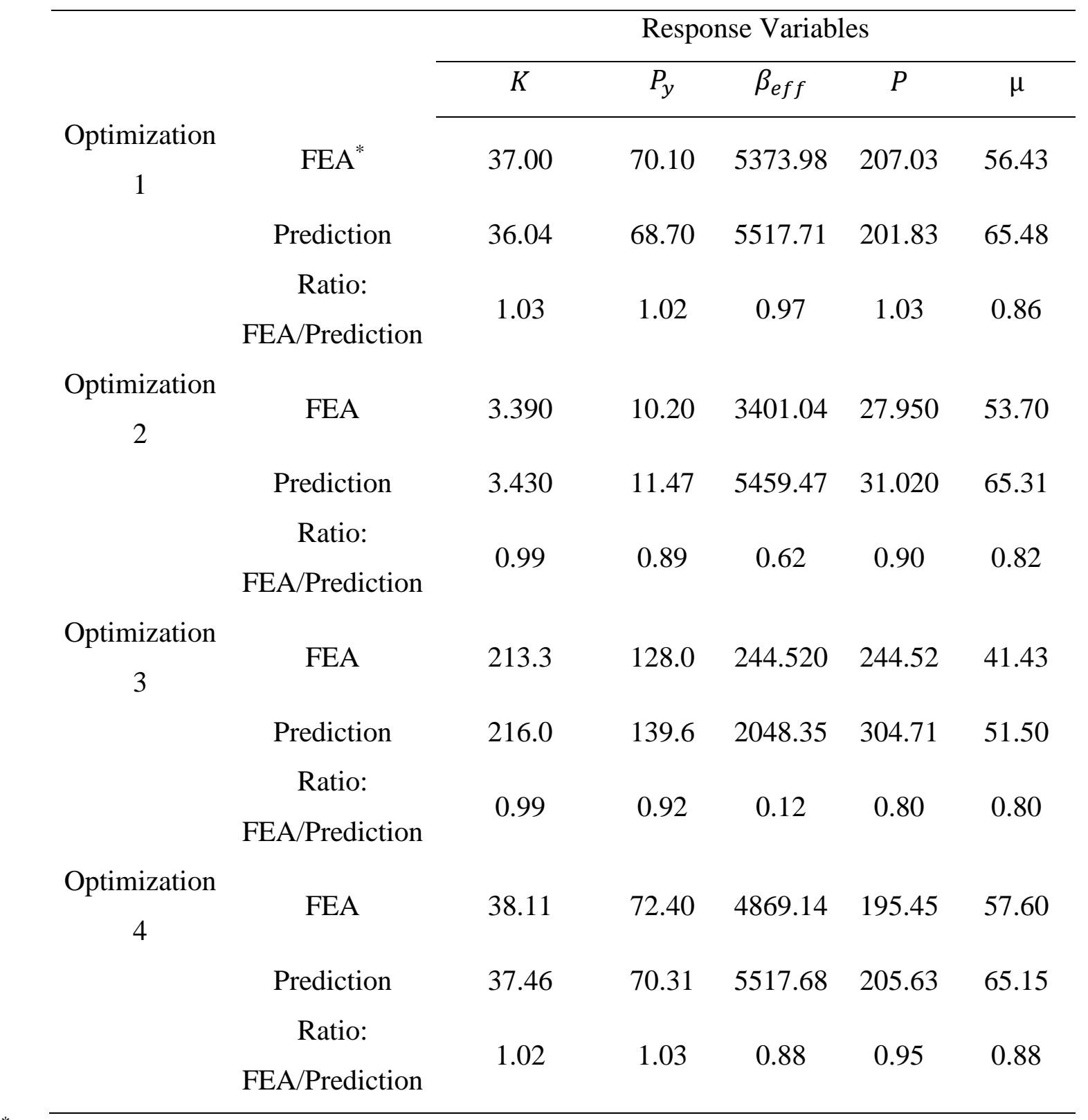

${ }^{*}$ FEA: FE analysis 


\section{CHAPTER 7 \\ SUMMARY AND CONCLUSIONS}

\subsection{Summary}

In this thesis, first, the effects of seven different design parameters on the cyclic response of steel plate fuses were examined. These factors included geometry and material related properties. The geometry related factors included the length, mid-width, end-width, thickness, and the band zone length of the fuse link. The material related factors included the yield strength and modulus of elasticity of the steel plate. A sensitivity analysis was conducted using a fractional factorial design-of-experiment method to statistically investigate the potential effects of the design factors as well as any significant interactions between the main factors. Later, ANSYS Mechanical APDL was used to develop 3D FE models and simulate the cyclic and monotonic response of steel plate fuses. The models were then validated against past experimental test results. A two-level, one-fourth, fractional factorial design was used to generate thirty-two factor combinations, which resulted in a sampling database for the sensitivity study. From the FE analysis of the models for these factor combinations, the cyclic load-displacement response of steel plate fuses was evaluated in terms of initial stiffness, effective damping, ductility, load capacity, and yield strength.

Following the sensitivity study, the response characteristics were studied in terms the ratios the of four influential factors. These factors were length, mid-width, end-width and thickness of steel plate fuses. The studied ratios were the length to end-width, end-width to thickness and midwidth to end-width ratio. A central composite design (CCD) was used for the design factors combinations. A total of 15 models were simulated in ANSYS Mechanical APDL. The cyclic loaddisplacement response of each model was extracted for the calculation of response variables. Predictive equations corresponding to each response variable were derived in terms of independent input parameters. To assess the accuracy of the predictive equations, seven additional model runs were carried out with randomly generated values over the range of each design factor. The accuracy of the predictive equations was confirmed by comparing the predicted response and simulation results.

The results obtained from the RSM were then used for the optimization of cyclic response characteristics of energy dissipating steel plate fuses. The optimum regions of each factor setting were determined using a desirability approach considering different optimization objectives. Four 
optimization problems were studied, including the maximization of effective damping and ductility; minimization of yield strength and maximization of effective damping and ductility; initial stiffness, effective damping and ductility; and maximum strength, effective damping and ductility. The predictions of optimum regions were also confirmed by simulations in ANSYS.

\subsection{Conclusions}

\subsubsection{Cyclic Response Sensitivity of Energy Dissipating Steel Plate Fuses}

Based on the obtained results of the sensitivity study the following conclusions are made:

- The fuse link end-width and thickness significantly influenced all the response characteristics. The fuse length and mid-width were influential on some of the response variables.

- By varying modulus of elasticity (from $185000 \mathrm{MPa}$ to $212000 \mathrm{MPa}$ ), steel yield strength (from $245 \mathrm{MPa}$ to $340 \mathrm{MPa}$ ), and the band zone length (from $5 \mathrm{~mm}$ to $75 \mathrm{~mm}$ ), it was observed that the cyclic performance of steel plate fuses was not significantly affected by the variation in these factors. The sensitivity analysis showed that the variability of modulus of elasticity, steel yield strength, band zone in the response quantities was generally below $1 \%$ except for the effective damping and ductility having percentage contributions close to or less than $5 \%$.

- The initial stiffness of a steel plate fuse was mostly influenced by the end-width, thickness, length and their interactions. These parameters accounted for approximately $92 \%$ of the total variability in the initial stiffness response. Further, the length and its interactions with the thickness and end-width had a negative effect on the initial stiffness of steel plate fuses.

- The yield strength of steel plate fuses was mostly influenced by the fuse link end-width, thickness, mid-width, and length. These factors accounted for about $87 \%$ of the total variability in the response. The effect of end-width and thickness and their interaction was noticeable among all these factors having a combined percentage contribution of approximately $75 \%$. Further, except for the fuse length all other factors had a positive effect on the yield strength response of steel plate fuses.

- The three important factors that significantly impacted the ultimate stiffness response were the end-width, thickness, and length. These factors with their interactions accounted for about $65 \%$ of the total response variability. End-width, thickness and their interaction had 
positive effects while the length and its interaction with the plate thickness had negative effects on the ultimate stiffness response.

- The effective damping of a steel plate fuse was mainly sensitive to the thickness and the end-width having a combined contribution of more than 50\%. A higher effective damping was observed for thicker steel plate fuses with a smaller end-width. The effective damping was increased by about 5 times for thick fuses with a smaller end-width.

- The factors that mostly influenced the load capacity of steel plate fuses were the thickness, end-width, mid-width, and length. These factors with their interactions accounted nearly for $94 \%$ of the entire variability in the response. Among these, over $80 \%$ of the load capacity was controlled by the thickness, end-width and their interactions. Further, the length and its interaction with thickness showed a negative effect on the load capacity response while all the other factors had positive effects. In addition, with increasing the end-width for thick fuses, the load capacity was increased significantly.

- The most important factors and interactions influencing the ductility of steel plate fuses were the thickness, length, end-width, the band zone length, as well as some interactions that had less than 5\% contributions. Overall, these factors and interactions accounted for about $85 \%$ of the total variability in the response. Among these important factors, the length and thickness were found to control over $50 \%$ of the ductility response variability. Further, it was observed that shorter and thicker fuses possess greater ductility.

\subsubsection{Predictive Equations and Multiple-response Optimization}

The following conclusions are made based on the predictive equations and optimization results:

- Moderate ranges of mid-width to end-width ratios $(0.35-0.86)$, moderate to higher ratios of length to end-width (3.5 - 6), and lower and higher ranges of steel plate end-width to thickness ratios (i.e., values smaller than 5.5 and values greater than 14) were more beneficial in terms of maximizing ductility and effective damping of energy dissipating steel plate fuses.

- Simultaneous minimization of the yield strength, and maximization of the effective damping and ductility was observed with moderate ranges of mid-width to end-width (0.33-0.95) and moderate to higher ranges of length to end-width ratios (3.73-6.00). 
Varying end-width to thickness (3.00-15.0) was not effective in achieving greater desirability values.

- Steel plate fuses with a mid-width to thickness ratio ranging from $(0.38-0.82 \mathrm{~mm})$, endwidth to thickness ratio (3.00-4.03), and length to end-width ratios from (2.00-4.45) were more desirable in maximizing the initial stiffness, effective damping, and ductility.

- Low values of mid-width to thickness $(3.00-4.35)$, moderate quantities of mid-width to end-width $(0.37-0.89)$ were desirable in reaching maximized values of effective damping, maximum strength and ductility. Varying length to mid-width values (2.64-6.00) was not effective in achieving greater desirability values.

\subsection{Future Recommendations}

The following points are recommended for analyzing the structural behavior of steel shear plate fuse links in future research:

- In this study, for the predictive equations and multiple-response optimization, a constant value of end-width was considered in all three significant ratios namely, length to end-width, end-width to thickness, and mid-width to end-width. Varying values of end-width with respect to all ratios should be also investigated to check the possible effects on each response variable.

- Other ratios such as length to thickness should also be investigated in further details to observe the potential effects of slenderness ratios on each response variable.

- The evaluation of systems such as rocking steel braced frame systems with the optimized shapes of steel plate fuse links should be comprehensively studied for future applications.

- The interaction of non-uniformity in thickness with other significant ratios should also be considered for future studies. 


\section{References}

[1] Juan Enrique Martı' nez-Rueda. On the Evolution of Energy Dissipation Devices for Seismic Design. Earthq Spectra 2002;18:309-46.

[2] Vargas R, Bruneau M. Experimental response of buildings designed with metallic structural fuses. II. J Struct Eng 2009;135:394-403. https://doi.org/10.1061/(ASCE)07339445(2009)135:4(394).

[3] Deierlein G, Krawinkler H, Ma X, Eatherton M, Hajjar J, Takeuchi T, et al. Earthquake resilient steel braced frames with controlled rocking and energy dissipating fuses. Steel Constr 2011;4:171-5. https://doi.org/10.1002/stco.201110023.

[4] Kurokawa Y, Sakamoto M, Yamada T, Kurino H, Kunisue A. Seismic design of a tall building with energy dissipation damper for the attenuation of torsional vibration. Struct Des Tall Build 1998;7:21-32. https://doi.org/10.1002/(SICI)10991794(199803)7:1<21::AID-TAL105>3.0.CO;2-8.

[5] Kelly JM, Skinner RI, Heine AJ. MECHANISMS OF ENERGY ABSORPTION IN SPECIAL DEVICES FOR USE IN EARTHQUAKE RESISTANT STRUCTURES. Bull New Zeal Soc Earthq Eng 1972;5:63-88.

[6] Lee CH, Lho SH, Kim DH, Oh J, Ju YK. Hourglass-shaped strip damper subjected to monotonic and cyclic loadings. Eng Struct 2016;119:122-34. https://doi.org/10.1016/j.engstruct.2016.04.019.

[7] Mualla IH, Belev B. Performance of steel frames with a new friction damper device under earthquake excitation. Eng Struct 2002;24:365-71. https://doi.org/10.1016/S01410296(01)00102-X.

[8] Bartera F, Giacchetti R. Steel dissipating braces for upgrading existing building frames. J Constr Steel Res 2004;60:751-69. https://doi.org/10.1016/S0143-974X(03)00141-X.

[9] Ma X, Borchers E, Pena A, Krawinkler H, Billington S, Deierlein GG. Design and Behavior of Steel Shear Plates with Openings as Energy-Dissipating Fuses. Intern. Report, John A. Blume Earthq. Eng. Center, Stanford Univ., 2011. 
[10] Chan RWK, Albermani F, Williams MS. Evaluation of yielding shear panel device for passive energy dissipation. J Constr Steel Res 2009;65:260-8.

https://doi.org/10.1016/j.jcsr.2008.03.017.

[11] Ghabraie K, Chan R, Huang X, Xie YM. Shape optimization of metallic yielding devices for passive mitigation of seismic energy. Eng Struct 2010;32:2258-67.

[12] Deng K, Pan P, Sun J, Liu J, Xue Y. Shape optimization design of steel shear panel dampers. J Constr Steel Res 2014;99:187-93.

[13] Ma X, Borchers E, Pena A, Krawinkler H, Billington S, Deierlein GG. Design and Behavior of Steel Shear Plates with Openings as Energy-Dissipating Fuses. Intern Report, John A Blume Earthq Eng Center, Stanford Univ 2011.

[14] Teruna DR, Majid TA, Budiono B. Experimental study of hysteretic steel damper for energy dissipation capacity. Adv Civ Eng 2015:1-12. https://doi.org/10.1155/2015/631726.

[15] Lee CH, Ju YK, Min JK, Lho SH, Kim SD. Non-uniform steel strip dampers subjected to cyclic loadings. Eng Struct 2015;99:192-204.

https://doi.org/10.1016/j.engstruct.2015.04.052.

[16] Farzampour A. Evaluating Shear Links for Use in Seismic Structural Fuses. Virginia Polytechnic Institute and State University, 2018. https://doi.org/10.1017/CBO9781107415324.004.

[17] Cortes G, Liu J, Cortés G. Steel Slit Panel Configurations. 14 th World Conf. Earthq. ..., 2008.

[18] Castiglioni CA, Kanyilmaz A. Numerical and Experimental Results of Project FUSEIS (Seismic Resistant Composite Steel Frames). 15th World Conf. Earthq. Eng., 2012.

[19] Hitaka T, Matsui C. Experimental study on steel shear wall with slits. J Struct Eng 2003;129. https://doi.org/10.1061/(ASCE)0733-9445(2003)129:5(586).

[20] Chan RWK, Albermani F. Experimental study of steel slit damper for passive energy dissipation. Eng Struct 2008;30:1058-66. https://doi.org/10.1016/j.engstruct.2007.07.005. 
[21] D.M. M. Steel Slit Panels for Lateral Resistance of Steel Frame Buildings. Purdue University West Lafayette, Indiana, 2006.

[22] T. H, C. M. Seismic performance of Steel Shear Wall with Slits integrated with multi story composite moment frame, International conference on behaviour of steel structures in seismic areas; Stessa 2006; 2006, p. 241-6.

[23] Oh SH, Kim YJ, Ryu HS. Seismic performance of steel structures with slit dampers. Eng Struct 2009;31:1997-2008. https://doi.org/10.1016/j.engstruct.2009.03.003.

[24] Köken A, Körołlu MA. Experimental study on beam-to-column connections of steel frame structures with steel slit dampers. J Perform Constr Facil 2015;29:1-11. https://doi.org/10.1061/(ASCE)CF.1943-5509.0000553.

[25] Nakamura. H. O, Sugiyama Y, Wada S, Baba M. A, Miyabara T. Passive control system using steel slit damper, part 1: test of steel slit damper. Summ. Tech. Pap. Annu. Meet. AIJ, Kanto, Japan: 1997, p. 843-4.

[26] M. A, A. H. Reduced Web Section Beams: Phase One Experimental Findings And Design Implications. Earthq Eng Online Arch 2002.

[27] Koppal M, Eatherton MR. Perforated steel plate shear walls for tunable seismic resistance. Struct. Congr. 2013 Bridg. Your Passion with Your Prof. - Proc. 2013 Struct. Congr., 2013.

[28] KAWAI Y, ONO T, SATO A, TOHNAI S, KONDO M. Allowable Design Formula for Steel Sheet Shear Walls with Burring Holes. 7th Int. Conf. Coupled Instab. Met. Struct., 2016, p. 1-12.

[29] Asselin RE, Fahnestock LA, Abrams DP. Behavior and Design of Fuse-Based Hybrid Masonry Seismic Structural Systems. 15th World Conf. Earthq. Eng. Lisbon Port., 2012.

[30] Andalib Z, Ali Kafi M, Bazzaz M, Momenzadeh S. Numerical evaluation of ductility and energy absorption of steel rings constructed from plates. Eng Struct 2018;169:94-106. https://doi.org/10.1016/j.engstruct.2018.05.034.

[31] Kobori T, Miura Y, Fukuzawa E, Yamada T, Arita T, Takenaka Y, et al. Development and 
application of hysteresis steel dampers, Balkema, Rotterdam: Earthquake Engineering, Tenth Conference; 1992, p. 1-6.

[32] Hanson R.D., Xia C. and SY. Design supplemental steel damping devices for buildings, Balkema, Rotterdam: Earthquake Engineering, Tenth Conference; 1992.

[33] Kobori T. Honeycomb Damper (HDS) 2017. http://www.koboritakken.co.jp/English/randd/ctrl41.html.

[34] Tagami J. Low cycle fatigue test of steel plate dampers with honeycomb shaped openings. Summ. Tech. Pap. Annu. Meet. AIJ, Chugoku, Japan: 1999, p. 791-6.

[35] Li HN, Li G. Experimental study of structure with "dual function" metallic dampers. Eng Struct 2007;29:1917-28. https://doi.org/10.1016/j.engstruct.2006.10.007.

[36] Li H, Huo L. Advances in structural control in civil engineering in China. Math Probl Eng 2010. https://doi.org/10.1155/2010/936081.

[37] Li G, Li HN. Experimental study and application in steel structure of "dual functions" metallic damper. Adv Steel Constr 2013;9:247-58.

[38] Farzampour A, Eatherton M. Parametric Study on Butterfly- Shaped Shear Links With Various Geometries. 11th US Natl Conf Earthq Eng 2018;24060.

[39] Farzampour A, Eatherton M. Investigating Limit States for Butterfly-Shaped and Straight Shear Links, 16ECEE, USA: 16th European Conference on Earthquake Engineering; 2018, p. 1-12.

[40] Farzampour A, Eatherton MR. Yielding and lateral torsional buckling limit states for butterfly-shaped shear links. Eng Struct 2019;180:442-51. https://doi.org/10.1016/j.engstruct.2018.10.040.

[41] Luth GP, Sargunuraj S, Krawinkler H, McDonald B. USC School of Cinema An Example of Reparable Performance Based Design, Menlo Park, California: SEAOC 2008 CONVENTION PROCEEDINGS; 2008, p. 1-20.

[42] Katayama T, Ito S, Kamura H, Ueki T, Okamoto H. Experimental Study on Hysteretic Damper With Low Yield Strength Steel Under Dynamic Loading, 2000, p. 1-8. 
[43] Moradi S, Burton H V. Response surface analysis and optimization of controlled rocking steel braced frames. Bull Earthq Eng 2018;16:4861-92. https://doi.org/10.1007/s10518018-0373-1.

[44] Moradi S, Burton H V., Kumar I. Parameterized fragility functions for controlled rocking steel braced frames. Eng Struct 2018;176:254-64. https://doi.org/10.1016/j.engstruct.2018.09.001.

[45] Dutta A. Design of Eccentrically Braced Frames. Https://EngineeringSfsuEdu/Download/Design_of_Eccentrically_Braced_FramesPdf 2008.

[46] El-Bahey S, Bruneau M. Structural fuse concept for bridges. 9th US Natl. 10th Can. Conf. Earthq. Eng. 2010, Incl. Pap. from 4th Int. Tsunami Symp., 2010.

[47] El-Bahey S, Bruneau M. Bridge piers with structural fuses and bi-steel columns. II: Analytical investigation. J Bridg Eng 2012;17:36-46. https://doi.org/10.1061/(ASCE)BE.1943-5592.0000236.

[48] ANSYS. ANSYS Mechanical APDL 2019.

[49] Hedayat AA. Prediction of the force displacement capacity boundary of an unbuckled steel slit damper. J Constr Steel Res 2015;114:30-50. https://doi.org/10.1016/j.jcsr.2015.07.003.

[50] Budaházy V, Dunai L. Parameter-refreshed Chaboche model for mild steel cyclic plasticity behaviour. Period Polytech Civ Eng 2013;57:139-55. https://doi.org/10.3311/PPci.7170.

[51] Chan RWK, Albermani F. Experimental study of steel slit damper for passive energy dissipation. Eng Struct 2008;30:1058-66. https://doi.org/10.1016/j.engstruct.2007.07.005.

[52] Ohsaki M, Nakajima T. Optimization of link member of eccentrically braced frames for maximum energy dissipation. J Constr Steel Res 2012;75:38-44. https://doi.org/10.1016/j.jcsr.2012.03.008.

[53] Hosseini Hashemi B, Ahmady Jazany R. Study of connection detailing on SMRF seismic 
behavior for unequal beam depths. J Constr Steel Res 2012;80:150-64.

https://doi.org/10.1016/j.jcsr.2011.08.005.

[54] Saffari H, Hedayat AA, Nejad MP. Post-Northridge connections with slit dampers to enhance strength and ductility. J Constr Steel Res 2013;80:138-52. https://doi.org/10.1016/j.jcsr.2012.09.023.

[55] Moradi S, Alam MS, Milani AS. Cyclic response sensitivity of post-tensioned steel connections using sequential fractional factorial design. J Constr Steel Res 2015;112:15566. https://doi.org/10.1016/j.jcsr.2015.04.022.

[56] DX10.0.7. Design-Expert® Software Version 92017.

[57] Montgomery D. Design and Analysis of Experiments. Eigth. Fowley, Donald; 2013.

[58] Mahmood Shah AS, Moradi S. Cyclic response sensitivity of energy dissipating steel plate fuses. Structures 2020;23:799-811. https://doi.org/10.1016/j.istruc.2019.12.026.

[59] Derringer G, Suich R. Simultaneous Optimization of Several Response Variables. J Qual Technol 1980;12:214-9. https://doi.org/10.1080/00224065.1980.11980968.

[60] Gunst RF, Myers RH, Montgomery DC. Response Surface Methodology: Process and Product Optimization Using Designed Experiments. Technometrics 1996;38:284-6. https://doi.org/10.2307/1270613. 